\title{
Antisymmetric Orbit Functions
}

\author{
Anatoliy KLIMYK ${ }^{\dagger}$ and Jiri PATERA \\ ${ }^{\dagger}$ Bogolyubov Institute for Theoretical Physics, 14-b Metrologichna Str., Kyiv 03143, Ukraine \\ E-mail: aklimyk@bitp.kiev.ua \\ $¥$ Centre de Recherches Mathématiques, Université de Montréal, \\ C.P.6128-Centre ville, Montréal, H3C 3J7, Québec, Canada \\ E-mail: patera@crm.umontreal.ca
}

Received December 25, 2006; Published online February 12, 2007

Original article is available at http://www.emis.de/journals/SIGMA/2007/023/

\begin{abstract}
In the paper, properties of antisymmetric orbit functions are reviewed and further developed. Antisymmetric orbit functions on the Euclidean space $E_{n}$ are antisymmetrized exponential functions. Antisymmetrization is fulfilled by a Weyl group, corresponding to a Coxeter-Dynkin diagram. Properties of such functions are described. These functions are closely related to irreducible characters of a compact semisimple Lie group $G$ of rank $n$. Up to a sign, values of antisymmetric orbit functions are repeated on copies of the fundamental domain $F$ of the affine Weyl group (determined by the initial Weyl group) in the entire Euclidean space $E_{n}$. Antisymmetric orbit functions are solutions of the corresponding Laplace equation in $E_{n}$, vanishing on the boundary of the fundamental domain $F$. Antisymmetric orbit functions determine a so-called antisymmetrized Fourier transform which is closely related to expansions of central functions in characters of irreducible representations of the group $G$. They also determine a transform on a finite set of points of $F$ (the discrete antisymmetric orbit function transform). Symmetric and antisymmetric multivariate exponential, sine and cosine discrete transforms are given.
\end{abstract}

Key words: antisymmetric orbit functions; signed orbits; products of orbits; orbit function transform; finite orbit function transform; finite Fourier transforms; finite cosine transforms; finite sine transforms; symmetric functions

2000 Mathematics Subject Classification: 33-02; 33E99; 42B99; 42C15; 58C40

\section{Introduction}

In [1] and [2] we considered properties and applications of symmetric orbit functions (they are called there orbit functions; without the word "symmetric"). They are closely related to finite groups $W$ of geometric symmetries generated by reflection transformations $r_{i}$ (that is, such that $\left.r_{i}^{2}=1\right), i=1,2, \ldots, n$, of the $n$-dimensional Euclidean space $E_{n}$ with respect to $(n-1)$ dimensional subspaces containing the origin. In order to obtain a symmetric orbit function we take a point $\lambda \in E_{n}$ and act upon $\lambda$ by all elements of the group $W$. If $O(\lambda)$ is the $W$-orbit of the point $\lambda$, that is the set of all different points of the form $w \lambda, w \in W$, then the symmetric orbit function, determined by $\lambda$, coincides with

$$
\phi_{\lambda}(x)=\sum_{\mu \in O(\lambda)} e^{2 \pi \mathrm{i}\langle\mu, x\rangle}
$$

where $\langle\mu, x\rangle$ is the scalar product on $E_{n}$. These functions are invariant with respect to the action by elements of the group $W: \phi_{\lambda}(w x)=\phi_{\lambda}(x), w \in W$. If $\lambda$ is an integral point of $E_{n}$, then $\phi_{\lambda}(x)$ is invariant with respect to the affine Weyl group $W^{\text {aff }}$ corresponding to $W$. Since in the simplest case symmetric orbit functions coincide with the cosine function, sometimes they are called $C$-functions. 
Symmetric orbit functions are multivariate functions having many beautiful and useful properties and applicable both in mathematics and engineering. For this reason, they can be treated as special functions [2].

Symmetricity is the main property of symmetric orbit functions, considered in [1] and [2], which make them useful in applications. Being a modification of monomial symmetric functions, they are directly related to the theory of symmetric (Laurent) polynomials [3, 4, 5, 6] (see Section 11 in [1]).

Symmetric orbit functions $\phi_{\lambda}(x)$ for integral $\lambda$ are closely related to the representation theory of compact groups $G$. In particular, they were effectively used for different calculations in representation theory $[7,8,9,10,11]$. They are constituents of traces (characters) of irreducible unitary representations of $G$. Although characters contain all (or almost all) information about the corresponding irreducible representations, they are seldom used as special functions. The reason is that a construction of characters is rather complicated, whereas orbit functions have much more simple structure.

The symmetric orbit function $\phi_{\lambda}(x)$ is a symmetrized (by means of the group $W$ ) exponential function $e^{2 \pi \mathrm{i}\langle\lambda, x\rangle}$ on $E_{n}$. For each transformation group $W$, the symmetric orbit functions form a complete orthogonal basis in the space of symmetric (with respect to $W$ ) polynomials in $e^{2 \pi \mathrm{i} x_{j}}, j=1,2, \ldots, n$, or in the Hilbert space obtained by closing this space with respect to an appropriate scalar product. Orbit functions $\phi_{\lambda}(x)$, when $\lambda$ runs over integral weights, determine so-called (symmetric) orbit function transform, which is a symmerization of the usual Fourier series expansion on $E_{n}$. If $\lambda$ runs over the space $E_{n}$, then $\phi_{\lambda}(x)$ determines a symmetric orbit function transform, which is a symmetrization of the usual continuous Fourier expansion in $E_{n}$ (that is, of the Fourier integral).

In the same way as the Fourier transform leads to discrete Fourier transform, the symmetric orbit function transform leads to a discrete analogue of this transform (which is a generalization of the discrete cosine transform [12]). This discrete transform is useful in many things related to discretization (see $[13,14,15,16,17,18,19,20]$ ). Construction of the discrete orbit function transform is fulfilled by means of the results of paper [21].

In this paper we consider antisymmetric orbit functions (since in the simplest case they coincide with the sine function, sometimes they are called $S$-functions). They are given by

$$
\varphi_{\lambda}(x)=\sum_{w \in W}(\operatorname{det} w) e^{2 \pi \mathrm{i}\langle w \lambda, x\rangle}, \quad x \in E_{n}
$$

where $\lambda$ is a strictly dominant weight and $\operatorname{det} w$ is a determinant of the transformation $w$ (it is equal to 1 or -1 , depending on either $w$ is a product of even or odd number of reflections). The orbit functions $\varphi_{\lambda}$ have many properties that the symmetric orbit functions $\phi_{\lambda}$ do. But antisymmetricity leads to some new properties which are useful for applications [22]. For integral $\lambda$, antisymmetric orbit functions are closely related to characters of irreducible representations of the corresponding compact Lie group $G$. Namely, the character $\chi_{\lambda}$ of the irreducible representation $T_{\lambda}, \lambda \in P_{+}$, coincides with $\varphi_{\lambda+\rho} / \varphi_{\rho}$, where $\rho$ is a half of a sum of positive roots related to the Weyl group $W$.

Symmetric orbit functions are a generalization of the cosine function, whereas antisymmetric orbit functions are a generalization of the sine function. A generalization of the exponential functions are called $E$-orbit functions. A detailed description of these functions for rank two see in [23].

Our goal in this paper is to bring together in full generality the diverse facts about antisymmetric orbit functions, many of which are not found in the literature, although they often are straightforward consequences of known facts. In general, for a given transformation group $W$ of the Euclidean space $E_{n}$, most of the properties of antisymmetric orbit functions, which are 
described in this paper, are implications of properties either of the orbits of the group $W$ or of the usual exponential function on $E_{n}$.

For dominant elements $\lambda$, antisymmetric orbit functions $\varphi_{\lambda}(x)$ are antisymmetric with respect to elements of the corresponding Weyl group, that is, $\varphi_{\lambda}(w x)=(\operatorname{det} w) \varphi_{\lambda}(x)$ for any $w \in W$. For this reason, antisymmetric orbit functions are defined only for strictly dominant elements $\lambda$ (a dominant element $\lambda$ is strictly dominant if $w \lambda=\lambda$ means that $w=1$ ). If $\lambda$ is integral strictly dominant element, then the corresponding antisymmetric orbit function $\varphi_{\lambda}(x)$ is antisymmetric also with respect to elements of the affine Weyl group $W^{\text {aff }}$, corresponding to the Weyl group $W$. Antisymmetricity is a main property of antisymmetric orbit functions. Because of antisymmetricity, it is enough to determine $\varphi_{\lambda}(x)$ only on a fundamental domain of the affine Weyl group $W^{\text {aff }}$ (if $\lambda$ is integral).

In the case when the group $W$ is a direct product of its subgroups, say $W=W_{1} \times W_{2}$, the fundamental domain is the Cartesian product of fundamental domains for $W_{1}$ and $W_{2}$. Similarly, antisymmetric orbit functions of $W$ are products of antisymmetric orbit functions of $W_{1}$ and $W_{2}$. Hence it suffices to carry out our considerations for groups $W$ which cannot be represented as a product of its subgroups (that is, for such $W$ for which a corresponding Coxeter-Dynkin diagram is connected).

In the article many examples of dimensions 2 and 3 are shown because they are likely to be used more often.

We shall need a general information on Weyl groups, affine Weyl groups, root systems and their properties. We have given this information in [1]. In order to make this paper self-contained we repeat a part of that information in Section 2.

In Section 3 we describe signed Weyl group orbits. They differ from the Weyl group orbits by a sign (equal to +1 or -1 ) assigned to each point of an orbit. To each signed orbit there corresponds an antisymmetric orbit function if a dominant element of the orbit is strictly dominant.

Section 4 is devoted to description of antisymmetric orbit functions. Antisymmetric orbit functions, corresponding to Coxeter-Dynkin diagrams, containing only two nodes, are given in an explicit form. In this section we also give explicit formulas for antisymmetric orbit functions, corresponding to Coxeter-Dynkin diagrams of $A_{n}, B_{n}, C_{n}$ and $D_{n}$, in the corresponding orthogonal coordinate systems. In Section 5 properties of antisymmetric orbit functions are described. If $\lambda$ is integral, then a main property of the antisymmetric orbit function $\varphi_{\lambda}$ is an invariance with respect to the affine Weyl group $W^{\text {aff }}$. We also give here the symmetric and antisymmetric orbit functions $\phi_{\rho}(x)$ and $\varphi_{\rho}(x)$, corresponding to the half-sum $\rho$ of positive roots, in a form of products of the cosine and sine functions of certain angles depending on $x$. Specific properties of antisymmetric orbit functions of the Coxeter-Dynkin diagram $A_{n}$ are given in Section 6 .

In Section 7 we consider expansions of products of symmetric (antisymmetric) orbit functions into a sum of symmetric or antisymmetric orbit functions. These expansions are closely related to properties of (signed) $W$-orbits. Many examples for expansions in the case of Coxeter-Dynkin diagrams $A_{2}$ and $C_{2}$ are considered. Section 8 is devoted to expansion of antisymmetric $W$-orbit functions into a sum of antisymmetric $W^{\prime}$-orbit functions, where $W^{\prime}$ is a subgroup of the Weyl group $W$. Many particular cases are studied in detail.

Connection between antisymmetric orbit functions $\varphi_{\lambda}(x)$ with integral $\lambda$ and characters of finite dimensional irreducible representations of the corresponding simple compact Lie groups is studied in Section 9. In particular, the well-known Weyl formula for characters of such representations contains antisymmetric orbit functions.

In Section 10 we expose antisymmetric orbit function transforms. There are two types of such transforms. The first one is an analogue of the expansion into Fourier series and the second one is an analogue of the Fourier integral transform. In Section 11 a description of an antisymmetric generalization of the multi-dimensional finite Fourier transform is given. This 
analogue is connected with grids on the corresponding fundamental domains for the affine Weyl groups $W^{\text {aff }}$. Symmetric and antisymmetric multivariate exponential discrete transforms, as well as symmetric and antisymmetric multivariate sine and cosine discrete transforms are given in this section.

In Section 12 we show that antisymmetric orbit functions are solutions of the Laplace equation on the corresponding $n$-dimensional simplex vanishing on a boundary of the simplex. It is shown that antisymmetric orbit functions are eigenfunctions of other differential operators.

Section 13 is devoted to exposition of symmetric and antisymmetric functions, which are symmetric and antisymmetric analogues of special functions of mathematical physics or orthogonal polynomials. In particular, we find eigenfunctions of antisymmetric and symmetric orbit function transforms. These eigenfunctions are connected with classical Hermite polynomials.

\section{Weyl groups and affine Weyl groups}

\subsection{Coxeter-Dynkin diagrams and Cartan matrices}

The sets of symmetric or antisymmetric orbit functions on the $n$-dimensional Euclidean space $E_{n}$ are determined by finite transformation groups $W$, generated by reflections $r_{i}, i=1,2, \ldots, n$ (a characteristic property of reflections is the equality $r_{i}^{2}=1$ ); the theory of such groups see, for example, in [24] and [25]. We are interested in those groups $W$ which are Weyl groups of semisimple Lie groups (semisimple Lie algebras). It is well-known that such Weyl groups together with the corresponding systems of reflections $r_{i}, i=1,2, \ldots, n$, are determined by Coxeter-Dynkin diagrams. There are 4 series and 5 separate simple Lie algebras, which uniquely determine their Weyl groups $W$. They are denoted as

$$
A_{n}(n \geq 1), \quad B_{n}(n \geq 3), \quad C_{n}(n \geq 2), \quad D_{n}(n \geq 4), \quad E_{6}, \quad E_{7}, \quad E_{8}, \quad F_{4}, G_{2} .
$$

To these Lie algebras there correspond connected Coxeter-Dynkin diagrams. To semisimple Lie algebras (they are direct sums of simple Lie subalgebras) there correspond Coxeter-Dynkin diagrams, which consist of connected parts, corresponding to simple Lie subalgebras; these parts are not connected with each other (a description of the correspondence between simple Lie algebras and Coxeter-Dynkin diagrams see, for example, in [26]). Thus, we describe only Coxeter-Dynkin diagrams, corresponding to simple Lie algebras. They are of the form
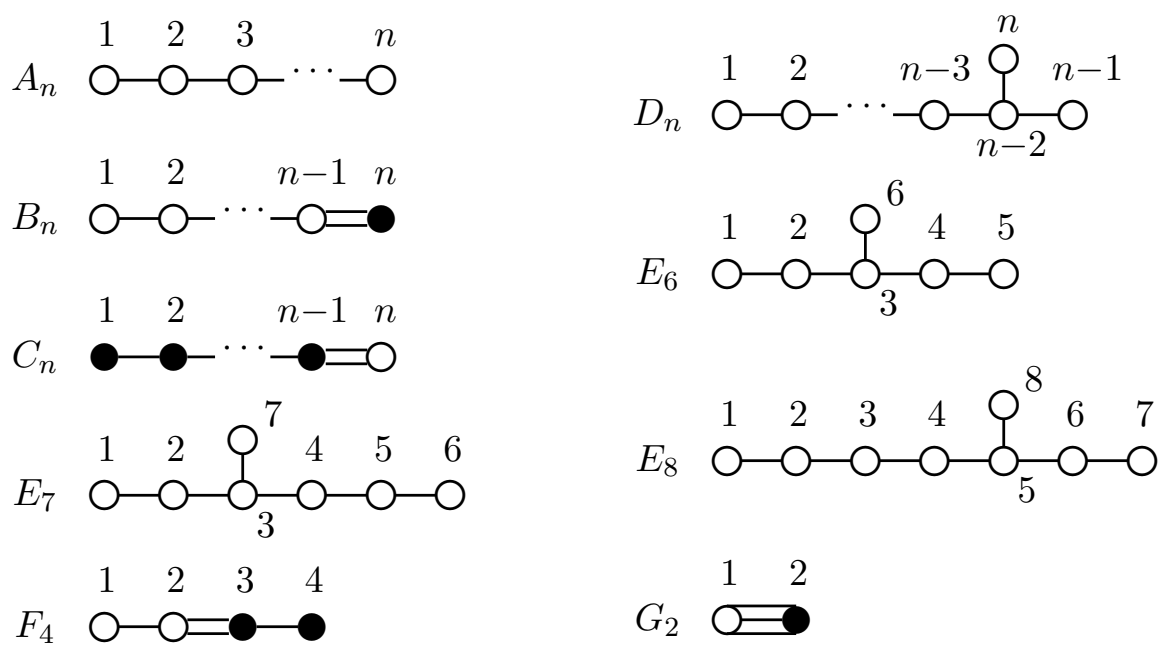

A diagram determines a certain non-orthogonal basis $\left\{\alpha_{1}, \alpha_{2}, \ldots, \alpha_{n}\right\}$ in the Euclidean space $E_{n}$. Each node is associated with a basis vector $\alpha_{k}$, called a simple root. A direct link between two nodes indicates that the corresponding basis vectors are not orthogonal. Conversely, 
an absence of a direct link between nodes implies orthogonality of the corresponding vectors. Single, double, and triple links indicate that the relative angles between the two simple roots are $2 \pi / 3,3 \pi / 4,5 \pi / 6$, respectively. There can be only two cases: all simple roots are of the same length or there are only two different lengths of simple roots. In the first case all simple roots are denoted by white nodes. In the case of two lengths, shorter roots are denoted by black nodes and longer ones by white nodes. Lengths of roots are determined uniquely up to a common constant. For the cases $B_{n}, C_{n}$, and $F_{4}$, the squared longer root length is double the squared shorter root length. For $G_{2}$, the squared longer root length is triple the squared shorter root length.

If two nodes are connected by a single line, then the angle between the corresponding simple roots is $2 \pi / 3$. If nodes are connected by a double line, then the angle is $3 \pi / 4$. A triple line means that the angle is $5 \pi / 6$. Simple roots of the same length are orthogonal to each other or an angle between them is $2 \pi / 3$.

To each Coxeter-Dynkin diagram there corresponds a Cartan matrix $M$, consisting of the entries

$$
M_{j k}=\frac{2\left\langle\alpha_{j}, \alpha_{k}\right\rangle}{\left\langle\alpha_{k}, \alpha_{k}\right\rangle}, \quad j, k \in\{1,2, \ldots, n\},
$$

where $\langle x, y\rangle$ denotes the scalar product of $x, y \in E_{n}$. Cartan matrices of simple Lie algebras are given in many places (see, for example, [27]). We recall them here for ranks 2 and 3 because of their usage later on:

$$
\begin{aligned}
& A_{2}:\left(\begin{array}{cc}
2 & -1 \\
-1 & 2
\end{array}\right), \quad C_{2}:\left(\begin{array}{cc}
2 & -1 \\
-2 & 2
\end{array}\right), \quad G_{2}:\left(\begin{array}{cc}
2 & -3 \\
-1 & 2
\end{array}\right), \\
& A_{3}:\left(\begin{array}{ccc}
2 & -1 & 0 \\
-1 & 2 & -1 \\
0 & -1 & 2
\end{array}\right), \quad B_{3}:\left(\begin{array}{ccc}
2 & -1 & 0 \\
-1 & 2 & -2 \\
0 & -1 & 2
\end{array}\right), \quad C_{3}:\left(\begin{array}{ccc}
2 & -1 & 0 \\
-1 & 2 & -1 \\
0 & -2 & 2
\end{array}\right) \text {. }
\end{aligned}
$$

Lengths of the basis vectors $\alpha_{i}$ are fixed by the corresponding Coxeter-Dynkin diagram up to a constant. We adopt the standard choice in the Lie theory, namely

$$
\langle\alpha, \alpha\rangle=2
$$

for all simple roots of $A_{n}, D_{n}, E_{6}, E_{7}, E_{8}$ and for the longer simple roots of $B_{n}, C_{n}, F_{4}, G_{2}$.

\subsection{Weyl group}

A Coxeter-Dynkin diagram determines uniquely the corresponding transformation group of $E_{n}$, generated by reflections $r_{i}, i=1,2, \ldots, n$. These reflections correspond to simple roots $\alpha_{i}$, $i=1,2, \ldots, n$. Namely, the transformation $r_{i}$ corresponds to the simple root $\alpha_{i}$ and is the reflection with respect to $(n-1)$-dimensional linear subspace (hyperplane) of $E_{n}$ (containing the origin), orthogonal to $\alpha_{i}$. It is well-known that such reflections are given by the formula

$$
r_{i} x=x-\frac{2\left\langle x, \alpha_{i}\right\rangle}{\left\langle\alpha_{i}, \alpha_{i}\right\rangle} \alpha_{i}, \quad i=1,2, \ldots, n, \quad x \in E_{n} .
$$

Each reflection $r_{i}$ can be thought as attached to the $i$-th node of the corresponding diagram.

A finite group $W$, generated by the reflections $r_{i}, i=1,2, \ldots, n$, is called a Weyl group, corresponding to a given Coxeter-Dynkin diagram. If a Weyl group $W$ corresponds to a CoxeterDynkin diagram of a simple Lie algebra $L$, then this Weyl group is often denoted by $W(L)$. Properties of Weyl groups are well known (see [24] and [25]). The orders (numbers of elements) of Weyl groups are given by the formulas

$$
\left|W\left(A_{n}\right)\right|=(n+1) !, \quad\left|W\left(B_{n}\right)\right|=\left|W\left(C_{n}\right)\right|=2^{n} n !, \quad\left|W\left(D_{n}\right)\right|=2^{n-1} n !,
$$




$$
\begin{aligned}
& \left|W\left(E_{6}\right)\right|=51840, \quad\left|W\left(E_{7}\right)\right|=2903040, \quad\left|W\left(E_{8}\right)\right|=696729600, \\
& \left|W\left(F_{4}\right)\right|=1152, \quad\left|W\left(G_{2}\right)\right|=12 .
\end{aligned}
$$

In particular,

$$
\left|W\left(A_{2}\right)\right|=6, \quad\left|W\left(C_{2}\right)\right|=8, \quad\left|W\left(A_{3}\right)\right|=24, \quad\left|W\left(C_{3}\right)\right|=48 .
$$

\section{$2.3 \quad$ Roots and weights}

A Coxeter-Dynkin diagram determines a system of simple roots in the Euclidean space $E_{n}$. Acting by elements of the Weyl group $W$ upon simple roots we obtain a finite system of vectors, which is invariant with respect to $W$. A set of all these vectors is called a system of roots associated with a given Coxeter-Dynkin diagram. It is denoted by $R$. As we see, a system of roots $R$ is calculated from simple roots by a straightforward algorithm.

It is proved (see, for example, [26]) that roots of $R$ are linear combinations of simple roots with integral coefficients. Moreover, there exist no roots, which are linear combinations of $\alpha_{i}$, $i=1,2, \ldots, n$, both with positive and negative coefficients. Therefore, the set of roots $R$ can be represented as a union $R=R_{+} \cup R_{-}$, where $R_{+}$(respectively $R_{-}$) is the set of roots which are linear combination of simple roots with positive (negative) coefficients. The set $R_{+}$(the set $R_{-}$) is called a set of positive (negative) roots.

As mentioned above, a set $R$ of roots is invariant under the action of elements of the Weyl group $W(R)$. However, $w R_{+} \neq R_{+}$if $w$ is not a trivial element of $W$. The following proposition holds:

Proposition 1. A reflection $r_{i} \in W$, corresponding to a simple root $\alpha_{i}$, maps $\alpha_{i}$ into $-\alpha_{i}$ and reflects the set $R_{+} \backslash\left\{\alpha_{i}\right\}$ of all other roots of $R_{+}$onto itself.

Let $X_{\alpha}$ be the $(n-1)$-dimensional linear subspace (hyperplane) of $E_{n}$ which contains the origin and is orthogonal to the root $\alpha$. Clearly, $X_{\alpha}=X_{-\alpha}$. The set of reflections with respect to $X_{\alpha}, \alpha \in R_{+}$, coincides with the set of all reflections of the corresponding Weyl group $W$. The hyperplane $X_{\alpha}$ consists of all points $x \in E_{n}$ such that $\langle x, \alpha\rangle=0$.

The subspaces $X_{\alpha}, \alpha \in R_{+}$, split the Euclidean space $E_{n}$ into connected parts which are called Weyl chambers. A number of Weyl chambers coincides with the number of elements of the Weyl group $W$. Elements of the Weyl group permute Weyl chambers. A part of a Weyl chamber, which belongs to some hyperplane $X_{\alpha}$ is called a wall of this Weyl chamber. If for some element $x$ of a Weyl chamber we have $\langle x, \alpha\rangle=0$ for some root $\alpha$, then this point belongs to a wall. The Weyl chamber consisting of points $x$ such that

$$
\left\langle x, \alpha_{i}\right\rangle \geq 0, \quad i=1,2, \ldots, n,
$$

is called the dominant Weyl chamber. It is denoted by $D_{+}$. Elements of $D_{+}$are called dominant. If $\left\langle x, \alpha_{i}\right\rangle>0, i=1,2, \ldots, n$, then $x$ is called strictly dominant element.

The set $Q$ of all linear combinations

$$
Q=\left\{\sum_{i=1}^{n} a_{i} \alpha_{i} \mid a_{i} \in \mathbb{Z}\right\} \equiv \bigoplus_{i} \mathbb{Z} \alpha_{i}
$$

is called a root lattice corresponding to a given Coxeter-Dynkin diagram. Its subset

$$
Q_{+}=\left\{\sum_{i=1}^{n} a_{i} \alpha_{i} \mid a_{i}=0,1,2, \ldots\right\}
$$

is called a positive root lattice. 
To each root $\alpha \in R$ there corresponds the coroot $\alpha^{\vee}$ defined by the formula

$$
\alpha^{\vee}=\frac{2 \alpha}{\langle\alpha, \alpha\rangle} .
$$

It is easy to see that $\alpha^{\vee \vee}=\alpha$. The set $Q^{\vee}$ of all linear combinations

$$
Q^{\vee}=\left\{\sum_{i=1}^{n} a_{i} \alpha_{i}^{\vee} \mid a_{i} \in \mathbb{Z}\right\} \equiv \bigoplus_{i} \mathbb{Z} \alpha_{i}^{\vee}
$$

is called a coroot lattice corresponding to a given Coxeter-Dynkin diagram. The subset

$$
Q_{+}^{\vee}=\left\{\sum_{i=1}^{n} a_{i} \alpha_{i}^{\vee} \mid a_{i}=0,1,2, \ldots\right\}
$$

is called a positive coroot lattice.

As noted above, the set of simple roots $\alpha_{i}, i=1,2, \ldots, n$, form a basis of the space $E_{n}$. In addition to the $\alpha$-basis, it is convenient to introduce the so-called $\omega$-basis, $\omega_{1}, \omega_{2}, \ldots, \omega_{n}$ (also called the basis of fundamental weights). The two bases are dual to each other in the following sense:

$$
\frac{2\left\langle\alpha_{j}, \omega_{k}\right\rangle}{\left\langle\alpha_{j}, \alpha_{j}\right\rangle} \equiv\left\langle\alpha_{j}^{\vee}, \omega_{k}\right\rangle=\delta_{j k}, \quad j, k \in\{1,2, \ldots, n\} .
$$

The $\omega$-basis (as well as the $\alpha$-basis) is not orthogonal.

Note that the factor $2 /\left\langle\alpha_{j}, \alpha_{j}\right\rangle$ can take only three values. Indeed, with the standard normalization of root lengths, we have

$$
\begin{aligned}
& \frac{2}{\left\langle\alpha_{k}, \alpha_{k}\right\rangle}=1 \quad \text { for roots of } A_{n}, D_{n}, E_{6}, E_{7}, E_{8}, \\
& \frac{2}{\left\langle\alpha_{k}, \alpha_{k}\right\rangle}=1 \quad \text { for long roots of } B_{n}, C_{n}, F_{4}, G_{2}, \\
& \frac{2}{\left\langle\alpha_{k}, \alpha_{k}\right\rangle}=2 \text { for short roots of } B_{n}, C_{n}, F_{4}, \\
& \frac{2}{\left\langle\alpha_{k}, \alpha_{k}\right\rangle}=3 \quad \text { for short roots of } G_{2} .
\end{aligned}
$$

For this reason, we get

$$
\begin{array}{ll}
\alpha_{k}^{\vee}=\alpha_{k} & \text { for roots of } A_{n}, D_{n}, E_{6}, E_{7}, E_{8}, \\
\alpha_{k}^{\vee}=\alpha_{k} & \text { for long roots of } B_{n}, C_{n}, F_{4}, G_{2}, \\
\alpha_{k}^{\vee}=2 \alpha_{k} & \text { for short roots of } B_{n}, C_{n}, F_{4}, \\
\alpha_{k}^{\vee}=3 \alpha_{k} & \text { for short roots of } G_{2} .
\end{array}
$$

The $\alpha$ - and $\omega$-bases are related by the Cartan matrix (2.1) and by its inverse:

$$
\alpha_{j}=\sum_{k=1}^{n} M_{j k} \omega_{k}, \quad \omega_{j}=\sum_{k=1}^{n}\left(M^{-1}\right)_{j k} \alpha_{k} .
$$

For ranks 2 and 3 the inverse Cartan matrices are of the form

$$
A_{2}: \frac{1}{3}\left(\begin{array}{cc}
2 & 1 \\
1 & 2
\end{array}\right), \quad C_{2}:\left(\begin{array}{cc}
1 & 1 / 2 \\
1 & 1
\end{array}\right), \quad G_{2}:\left(\begin{array}{cc}
2 & 3 \\
1 & 2
\end{array}\right),
$$




$$
A_{3}: \frac{1}{4}\left(\begin{array}{ccc}
3 & 2 & 1 \\
2 & 4 & 2 \\
1 & 2 & 3
\end{array}\right), \quad B_{3}: \frac{1}{2}\left(\begin{array}{ccc}
2 & 2 & 2 \\
2 & 4 & 4 \\
1 & 2 & 3
\end{array}\right), \quad C_{3}: \frac{1}{2}\left(\begin{array}{ccc}
2 & 2 & 1 \\
2 & 4 & 2 \\
2 & 4 & 3
\end{array}\right)
$$

Later on we need to calculate scalar products $\langle x, y\rangle$ when $x$ and $y$ are given by coordinates $x_{i}$ and $y_{i}$ in $\omega$-basis. It is given by the formula

$$
\langle x, y\rangle=\frac{1}{2} \sum_{j, k=1}^{n} x_{j} y_{k}\left(M^{-1}\right)_{j k}\left\langle\alpha_{k} \mid \alpha_{k}\right\rangle=x M^{-1} D y^{T}=x S y^{T}
$$

where $D$ is the diagonal matrix $\operatorname{diag}\left(\frac{1}{2}\left\langle\alpha_{1}, \alpha_{1}\right\rangle, \ldots, \frac{1}{2}\left\langle\alpha_{n}, \alpha_{n}\right\rangle\right)$. Matrices $S$, called 'quadratic form matrices', are shown in [27] for all connected Coxeter-Dynkin diagrams.

The sets $P$ and $P_{+}$, defined as

$$
P=\mathbb{Z} \omega_{1}+\cdots+\mathbb{Z} \omega_{n} \supset P_{+}=\mathbb{Z}^{\geq 0} \omega_{1}+\cdots+\mathbb{Z}^{\geq 0} \omega_{n}
$$

are called respectively the weight lattice and the cone of dominant weights. The set $P$ can be characterized as a set of all $\lambda \in E_{n}$ such that

$$
\frac{2\left\langle\alpha_{j}, \lambda\right\rangle}{\left\langle\alpha_{j}, \alpha_{j}\right\rangle}=\left\langle\alpha_{j}^{\vee}, \lambda\right\rangle \in \mathbb{Z}
$$

for all simple roots $\alpha_{j}$. Clearly, $Q \subset P$. Below we shall need also the set $P_{+}^{+}$of dominant weights of $P_{+}$, which do not belong to any Weyl chamber (the set of integral strictly dominant weights). Then $\lambda \in P_{+}^{+}$means that $\left\langle\lambda, \alpha_{i}\right\rangle>0$ for all simple roots $\alpha_{i}$. We have

$$
P_{+}^{+}=\mathbb{Z}^{>0} \omega_{1}+\mathbb{Z}^{>0} \omega_{2}+\cdots+\mathbb{Z}^{>0} \omega_{n}
$$

The smallest dominant weights of $P_{+}$, different from zero, coincide with the elements $\omega_{1}, \omega_{2}$, $\ldots, \omega_{n}$ of the $\omega$-basis. They are called fundamental weights. They are highest weights of fundamental irreducible representations of the corresponding simple Lie algebra $L$.

Through the paper we often use the following notation for weights in $\omega$-basis:

$$
z=\sum_{j=1}^{n} a_{j} \omega_{j}=\left(\begin{array}{llll}
a_{1} & a_{2} & \ldots & a_{n}
\end{array}\right), \quad a_{1}, \ldots, a_{n} \in \mathbb{Z}
$$

If $x=\sum_{j=1}^{n} b_{j} \alpha_{j}^{\vee}$, then

$$
\langle z, x\rangle=\sum_{j=1}^{n} a_{j} b_{j}
$$

\subsection{Highest root}

There exists a unique highest (long) root $\xi$ and a unique highest short root $\xi_{s}$. The highest (long) root can be written as

$$
\xi=\sum_{i=1}^{n} m_{i} \alpha_{i}=\sum_{i=1}^{n} m_{i} \frac{\left\langle\alpha_{i}, \alpha_{i}\right\rangle}{2} \alpha_{i}^{\vee} \equiv \sum_{i=1}^{n} q_{i} \alpha_{i}^{\vee} .
$$

The coefficients $m_{i}$ and $q_{i}$ can be viewed as attached to the $i$-th node of the diagram. They are called marks and comarks and are often listed in the literature (see, for example, [27]). In root 
systems with two lengths of roots, namely in $B_{n}, C_{n}, F_{4}$ and $G_{2}$, the highest (long) root $\xi$ is of the form

$$
\begin{aligned}
& B_{n}: \xi=(010 \ldots 0)=\alpha_{1}+2 \alpha_{2}+2 \alpha_{3}+\cdots+2 \alpha_{n}, \\
& C_{n}: \xi=(20 \ldots 0)=2 \alpha_{1}+2 \alpha_{2}+\cdots+2 \alpha_{n-1}+\alpha_{n} \text {, } \\
& F_{4}: \xi=(1000)=2 \alpha_{1}+3 \alpha_{2}+4 \alpha_{3}+2 \alpha_{4} \text {, } \\
& G_{2}: \xi=\quad(10)=2 \alpha_{1}+3 \alpha_{2} \text {. }
\end{aligned}
$$

For $A_{n}, D_{n}$, and $E_{n}$, all roots are of the same length, hence $\xi_{s}=\xi$. We have

$$
\begin{aligned}
& A_{n}: \xi=(10 \ldots 01)=\alpha_{1}+\alpha_{2}+\cdots+\alpha_{n}, \\
& D_{n}: \xi=(010 \ldots 0)=\alpha_{1}+2 \alpha_{2}+\cdots+2 \alpha_{n-2}+\alpha_{n-1}+\alpha_{n} \text {, } \\
& E_{6}: \xi=(010 \ldots 0)=\alpha_{1}+2 \alpha_{2}+3 \alpha_{3}+2 \alpha_{4}+\alpha_{5}+2 \alpha_{6} \text {, } \\
& E_{7}: \xi=(100 \ldots 0)=2 \alpha_{1}+3 \alpha_{2}+4 \alpha_{3}+3 \alpha_{4}+2 \alpha_{5}+\alpha_{6}+2 \alpha_{7} \text {, } \\
& E_{8}: \quad \xi=(00 \ldots 01)=2 \alpha_{1}+3 \alpha_{2}+4 \alpha_{3}+5 \alpha_{4}+6 \alpha_{5}+4 \alpha_{6}+2 \alpha_{7}+3 \alpha_{8} \text {. }
\end{aligned}
$$

Note that for highest root $\xi$ we have

$$
\xi^{\vee}=\xi
$$

Moreover, if all simple roots are of the same length, then

$$
\alpha_{i}^{\vee}=\alpha_{i}
$$

For this reason,

$$
\left(q_{1}, q_{2}, \ldots, q_{n}\right)=\left(m_{1}, m_{2}, \ldots, m_{n}\right)
$$

for $A_{n}, D_{n}$ and $E_{n}$. Formulas (2.13)-(2.18) determine these numbers. For short roots $\alpha_{i}$ of $B_{n}$, $C_{n}$ and $F_{4}$ we have $\alpha_{i}^{\vee}=2 \alpha_{i}$. For short root $\alpha_{2}$ of $G_{2}$ we have $\alpha_{2}^{\vee}=3 \alpha_{2}$. For this reason,

$$
\begin{aligned}
& \left(q_{1}, q_{2}, \ldots, q_{n}\right)=(1,2, \ldots, 2,1) \text { for } B_{n}, \\
& \left(q_{1}, q_{2}, \ldots, q_{n}\right)=(1,1, \ldots, 1,1) \text { for } C_{n}, \\
& \left(q_{1}, q_{2}, q_{3}, q_{4}\right)=(2,3,2,1) \text { for } F_{4}, \\
& \left(q_{1}, q_{2}\right)=(2,1) \quad \text { for } G_{2} .
\end{aligned}
$$

To each root system $R$ there corresponds an extended root system (which is also called an affine root system). It is constructed with the help of the highest root $\xi$ of $R$. Namely, if $\alpha_{1}, \alpha_{2}, \ldots, \alpha_{n}$ is a set of all simple roots, then the roots

$$
\alpha_{0}:=-\xi, \alpha_{1}, \alpha_{2}, \ldots, \alpha_{n}
$$

constitute a set of simple roots of the corresponding extended root system. Taking into account the orthogonality (non-orthogonality) of the root $\alpha_{0}$ to other simple roots, the diagram of an extended root system can be constructed (which is an extension of the corresponding CoxeterDynkin diagram; see, for example, [28]). Note that for all simple Lie algebras (except for $A_{n}$ ) only one simple root is orthogonal to the root $\alpha_{0}$. In the case of $A_{n}$, the two simple roots $\alpha_{1}$ and $\alpha_{n}$ are not orthogonal to $\alpha_{0}$. 


\subsection{Affine Weyl groups}

We are interested in antisymmetric orbit functions which are given on the Euclidean space $E_{n}$. These functions are anti-invariant with respect to action by elements of a Weyl group $W$, which is a transformation group of $E_{n}$. However, $W$ does not describe all symmetries of orbit functions corresponding to weights $\lambda \in P_{+}^{+}$. A whole group of anti-invariances of antisymmetric orbit functions is isomorphic to the affine Weyl group $W^{\text {aff }}$ which is an extension of the Weyl group $W$. This group is defined as follows.

Let $\alpha_{1}, \alpha_{2}, \ldots, \alpha_{n}$ be simple roots in the Euclidean space $E_{n}$ and let $W$ be the corresponding Weyl group. The group $W$ is generated by reflections $r_{\alpha_{i}}, i=1,2, \ldots, n$. In order to construct the affine Weyl group $W^{\text {aff }}$, corresponding to the group $W$, we have to add an additional reflection. This reflection is constructed as follows.

We consider the reflection $r_{\xi}$ with respect to the $(n-1)$-dimensional subspace (hyperplane) $X_{n-1}$ containing the origin and orthogonal to the highest (long) root $\xi$, given in (2.8):

$$
r_{\xi} x=x-\frac{2\langle x, \xi\rangle}{\langle\xi, \xi\rangle} \xi
$$

Clearly, $r_{\xi} \in W$. We shift the hyperplane $X_{n-1}$ by the vector $\xi^{\vee} / 2$, where $\xi^{\vee}=2 \xi /\langle\xi, \xi\rangle$. (Note that by (2.18) we have $\xi^{\vee}=\xi$. However, it is convenient to use here $\xi^{\vee}$.) The reflection with respect to the hyperplane $X_{n-1}+\xi^{\vee} / 2$ will be denoted by $r_{0}$. Then in order to fulfill the transformation $r_{0}$ we have to fulfill the transformation $r_{\xi}$ and then to shift the result by $\xi^{\vee}$, that is,

$$
r_{0} x=r_{\xi} x+\xi^{\vee} .
$$

We have $r_{0} 0=\xi^{\vee}$ and it follows from (2.19) that $r_{0}$ maps $x+\xi^{\vee} / 2$ to

$$
r_{\xi}\left(x+\xi^{\vee} / 2\right)+\xi^{\vee}=x+\xi^{\vee} / 2-\left\langle x, \xi^{\vee}\right\rangle \xi \text {. }
$$

Therefore,

$$
\begin{aligned}
r_{0}\left(x+\xi^{\vee} / 2\right) & =x+\xi^{\vee} / 2-\frac{2\langle x, \xi\rangle}{\langle\xi, \xi\rangle} \xi=x+\xi^{\vee} / 2-\frac{2\left\langle x, \xi^{\vee}\right\rangle}{\left\langle\xi^{\vee}, \xi^{\vee}\right\rangle} \xi^{\vee} \\
& =x+\xi^{\vee} / 2-\frac{2\left\langle x+\xi^{\vee} / 2, \xi^{\vee}\right\rangle}{\left\langle\xi^{\vee}, \xi^{\vee}\right\rangle} \xi^{\vee}+\frac{2\left\langle\xi^{\vee} / 2, \xi^{\vee}\right\rangle}{\left\langle\xi^{\vee}, \xi^{\vee}\right\rangle} \xi^{\vee} .
\end{aligned}
$$

Denoting $x+\xi^{\vee} / 2$ by $y$ we obtain that $r_{0}$ is given also by the formula

$$
r_{0} y=y+\left(1-\frac{2\left\langle y, \xi^{\vee}\right\rangle}{\left\langle\xi^{\vee}, \xi^{\vee}\right\rangle}\right) \xi^{\vee}=\xi^{\vee}+r_{\xi} y .
$$

The element $r_{0}$ does not belong to $W$ since elements of $W$ do not move the point $0 \in E_{n}$.

The hyperplane $X_{n-1}+\xi^{\vee} / 2$ coincides with the set of points $y$ such that $r_{0} y=y$. It follows from (2.20) that this hyperplane is given by the equation

$$
1=\frac{2\left\langle y, \xi^{\vee}\right\rangle}{\left\langle\xi^{\vee}, \xi^{\vee}\right\rangle}=\langle y, \xi\rangle=\sum_{k=1}^{n} a_{k} q_{k},
$$

where

$$
y=\sum_{k=1}^{n} a_{k} \omega_{k}, \quad \xi=\sum_{k=1}^{n} q_{k} \alpha_{k}^{\vee}
$$

(see (2.7)). 
A group of transformations of the Euclidean space $E_{n}$ generated by reflections $r_{0}, r_{\alpha_{1}}, \ldots, r_{\alpha_{n}}$ is called the affine Weyl group of the root system $R$ and is denoted by $W^{\text {aff }}$ or by $W_{R}^{\text {aff }}$ (if is necessary to indicate the initial root system), see [28]. $W^{\text {aff }}$.

Adjoining the reflection $r_{0}$ to the Weyl group $W$ completely change properties of the group

If $r_{\xi}$ is the reflection with respect to the hyperplane $X_{n-1}$, then due to (2.19) and (2.20) for any $x \in E_{n}$ we have

$$
r_{0} r_{\xi} x=r_{0}\left(r_{\xi} x\right)=\xi^{\vee}+r_{\xi} r_{\xi} x=x+\xi^{\vee} .
$$

Clearly, $\left(r_{0} r_{\xi}\right)^{k} x=x+k \xi^{\vee}, k=0, \pm 1, \pm 2, \ldots$, that is, the set of elements $\left(r_{0} r_{\xi}\right)^{k}, k=$ $0, \pm 1, \pm 2, \ldots$, is an infinite commutative subgroup of $W^{\text {aff }}$. This means that (unlike to the Weyl group $W$ ) $W^{\text {aff }}$ is an infinite group.

Since $r_{0} 0=\xi^{\vee}$, for any $w \in W$ we have

$$
w r_{0} 0=w \xi^{\vee}=\xi_{w}^{\vee}
$$

where $\xi_{w}^{\vee}$ is a coroot of the same length as the coroot $\xi^{\vee}$. For this reason, $w r_{0}$ is the reflection with respect to the $(n-1)$-hyperplane perpendicular to the root $\xi_{w}^{\vee}$ and containing the point $\xi_{w}^{\vee} / 2$. Moreover,

$$
\left(w r_{0}\right) r_{\xi_{w}} x=x+\xi_{w}^{\vee}
$$

We also have $\left(\left(w r_{0}\right) r_{\xi_{w}}\right)^{k} x=x+k \xi_{w}^{\vee}, k=0, \pm 1, \pm 2, \ldots$ Since $w$ is any element of $W$, then the set $w \xi^{\vee}, w \in W$, coincides with the set of coroots of $R^{\vee}$, corresponding to all long roots of the root system $R$. Thus, the set $W^{\text {aff }} \cdot 0$ coincides with the lattice $Q_{l}^{\vee}$ generated by coroots $\alpha^{\vee}$ taken for all long roots $\alpha$ from $R$.

It is checked for each type of root systems that each coroot $\xi_{s}^{\vee}$ for a short root $\xi_{s}$ of $R$ is a linear combination of coroots $w \xi^{\vee} \equiv \xi_{w}, w \in W$, with integral coefficients, that is, $Q^{\vee}=Q_{l}^{\vee}$. Therefore, The set $W^{\text {aff }} \cdot 0$ coincides with the coroot lattice $Q^{\vee}$ of $R$.

Let $\hat{Q}^{\vee}$ be the subgroup of $W^{\text {aff }}$ generated by the elements

$$
\left(w r_{0}\right) r_{w}, \quad w \in W
$$

where $r_{w} \equiv r_{\xi_{w}}$ for $w \in W$. Since elements (2.22) pairwise commute with each other (since they are shifts), $\hat{Q}^{\vee}$ is a commutative group. The subgroup $\hat{Q}^{\vee}$ can be identified with the coroot lattice $Q^{\vee}$. Namely, if for $g \in \hat{Q}^{\vee}$ we have $g \cdot 0=\gamma \in Q^{\vee}$, then $g$ is identified with $\gamma$. This correspondence is one-to-one.

The subgroups $W$ and $\hat{Q}^{\vee}$ generate $W^{\text {aff }}$ since a subgroup of $W^{\text {aff }}$, generated by $W$ and $\hat{Q}^{\vee}$, contains the element $r_{0}$. The group $W^{\text {aff }}$ is a semidirect product of its subgroups $W$ and $\hat{Q}^{\vee}$, where $\hat{Q}^{\vee}$ is an invariant subgroup (see Section 5.2 in [1] for details).

\subsection{Fundamental domain}

An open connected simply connected set $D \subset E_{n}$ is called a fundamental domain for the group $W^{\text {aff }}$ (for the group $W$ ) if it does not contains equivalent points (that is, points $x$ and $x^{\prime}$ such that $x=w x$ ) and if its closure contains at least one point from each $W^{\text {aff }}$-orbit (from each $W$-orbit). It is evident that the dominant Weyl chamber (without walls of this chamber) is a fundamental domain for the Weyl group $W$. Recall that this domain consists of all points $x=a_{1} \omega_{1}+\cdots+a_{n} \omega_{n} \in E_{n}$ for which

$$
a_{i}=\left\langle x, \alpha_{i}^{\vee}\right\rangle>0, \quad i=1,2, \ldots, n .
$$


We wish to describe a fundamental domain for the group $W^{\text {aff }}$. Since $W \subset W^{\text {aff }}$, it can be chosen as a subset of the dominant Weyl chamber for $W$.

We have seen that the element $r_{0} \in W^{\text {aff }}$ is a reflection with respect to the hyperplane $X_{n-1}+\xi^{\vee} / 2$, orthogonal to the root $\xi$ and containing the point $\xi^{\vee} / 2$. This hyperplane is given by the equation (2.21). This equation shows that the hyperplane $X_{n-1}+\xi^{\vee} / 2$ intersects the axices, determined by the vectors $\omega_{i}$, in the points $\omega_{i} / q_{i}, i=1,2, \ldots, n$, where $q_{i}$ are such as in (2.21). We create the simplex with $n+1$ vertices in the points

$$
0, \frac{\omega_{1}}{q_{1}}, \ldots, \frac{\omega_{n}}{q_{n}}
$$

By the definition of this simplex and by (2.21), this simplex consists of all points $y$ of the dominant Weyl chamber for which $\langle y, \xi\rangle \leq 1$. Clearly, the interior $F$ of this simplex belongs to the dominant Weyl chamber. The following theorem is true (see, for example, [1]):

Theorem 1. The set $F$ is a fundamental domain for the affine Weyl group $W^{\text {aff }}$.

For the rank 2 cases the fundamental domain is the interior of the simplex with the following vertices:

$$
\begin{aligned}
& A_{2}:\left\{0, \omega_{1}, \omega_{2}\right\}, \\
& C_{2}:\left\{0, \omega_{1}, \omega_{2}\right\}, \\
& G_{2}:\left\{0, \frac{\omega_{1}}{2}, \omega_{2}\right\}
\end{aligned}
$$

\section{$3 \quad$ Weyl group signed orbits}

\subsection{Signed orbits}

As we have seen, the $(n-1)$-dimensional linear subspaces $X_{\alpha}$ of $E_{n}$, orthogonal to positive roots $\alpha$ and containing the origin, divide the space $E_{n}$ into connected parts, which are called Weyl chambers. A number of such chambers is equal to an order of the corresponding Weyl group $W$. Elements of the Weyl group permute these chambers. There exists a single chamber $D_{+}$such that $\left\langle\alpha_{i}, x\right\rangle \geq 0, x \in D_{+}, i=1,2, \ldots, n$. It is the dominant Weyl chamber.

Clearly, the cone of dominant weights $P_{+}$belongs to the dominant Weyl chamber $D_{+}$. (Note that it is not a case for the set $Q_{+}$.) We have $P \cap D_{+}=P_{+}$.

Let $y$ be an arbitrary dominant element of the Euclidean space $E_{n}$, which does not lie on some Weyl chamber. We act upon $y$ by all elements of the Weyl group $W$. As a result, we obtain a set of elements $w y, w \in W$, which is called Weyl group orbit. All these elements are pairwise different. We attach to each point $w y$ a sign coinciding with a sign of $\operatorname{det} w$. The set of all points $w y, w \in W$, together with their signs is called a signed orbit of the point $y$ with respect the Weyl group (or a Weyl group signed orbit, containing y). Points of signed orbits will be denoted by $x^{+}$or $x^{-}$, depending on a sign. Sometimes, we denote points $w y, y \in D_{+}$, of the signed orbit, containing the point $y$, as $w y^{\operatorname{det} w}$, where instead of a sign we have +1 or -1 , respectively.

An orbit (where points do not have signs) of a point $y \in D_{+}$is denoted by $O(y)$ or $O_{W}(y)$. A size of an orbit $O(y)$ is a number $|O(y)|$ of its elements. Each Weyl chamber contains only one point of a fixed orbit $Q(y)$. A signed orbit of a strictly dominant point $y \in E_{n}$ is denoted by $O^{ \pm}(y)$ or $O_{W}^{ \pm}(y)$.

Note that orbits $O(y)$ are defined for any dominant elements $y \in E_{n}$. Signed orbits $O^{ \pm}(y)$ can be defined only for strictly dominant $y \in E_{n}$. 


\subsection{Signed orbits of $A_{1}, A_{1} \times A_{1}, A_{2}, C_{2}, G_{2}$}

Assuming that $a>0$ and $b>0$, we list the contents of signed orbits in $\omega$-basis:

$$
\begin{aligned}
A_{1}: & O^{ \pm}(a) \ni(a)^{+},(-a)^{-} \\
A_{1} \times A_{1}: & O^{ \pm}(a b) \ni(a b)^{+},(-a b)^{-},(a-b)^{-},(-a-b)^{+} \\
A_{2}: & O^{ \pm}(a b) \ni(a b)^{+},(-a a+b)^{-},(a+b-b)^{-}, \\
& (-b-a)^{-},(-a-b a)^{+},(b-a-b)^{+} .
\end{aligned}
$$

In the cases of $C_{2}$ and $G_{2}$ (where the second simple root is the longer one for $C_{2}$ and the shorter one for $G_{2}$ ) we have

$$
\begin{array}{cc}
C_{2}: & O^{ \pm}(a b) \ni(a b)^{+},(-a a+b)^{-},(a+2 b-b)^{-},(a+2 b-a-b)^{+}, \\
& (-a-b)^{+},(-a-a-b)^{-},(-a-2 b b)^{-},(-a-2 b a+b)^{+}, \\
G_{2}: & O^{ \pm}(a b) \ni \pm(a b)^{+}, \pm(-a 3 a+b)^{-}, \pm(a+b-b)^{-}, \\
& \pm(2 a+b-3 a-b)^{+}, \pm(-a-b 3 a+2 b)^{+}, \pm(-2 a-b 3 a+2 b)^{-},
\end{array}
$$

where $\pm(c, d)^{+}$means two signed points $(c, d)^{+}$and $(-c,-d)^{+}$.

As we see, for each point $(c d)$ of a signed orbit of $C_{2}$ or $G_{2}$ there exists in the orbit the point $(-c-d)$ with the same sign.

\subsection{The case of $A_{n}$}

In the cases of Coxeter-Dynkin diagrams $A_{n-1}, B_{n}, C_{n}, D_{n}$, root and weight lattices, Weyl groups and signed orbits are described in a simple way by using the orthogonal coordinate system in $E_{n}$. In particular, this coordinate system is useful under practical work with signed orbits.

In the case $A_{n}$ it is convenient to describe root and weight lattices, Weyl group and antisymmetric orbit functions in the subspace of the Euclidean space $E_{n+1}$, given by the equation

$$
x_{1}+x_{2}+\cdots+x_{n+1}=0
$$

where $x_{1}, x_{2}, \ldots, x_{n+1}$ are orthogonal coordinates of a point $x \in E_{n+1}$. The unit vectors in directions of these coordinates are denoted by $\mathbf{e}_{j}$, respectively. Clearly, $\mathbf{e}_{i} \perp \mathbf{e}_{j}, i \neq j$. The set of roots of $A_{n}$ is given by the vectors

$$
\alpha_{i j}=\mathbf{e}_{i}-\mathbf{e}_{j}, \quad i \neq j
$$

The roots

$$
\alpha_{i j}=\mathbf{e}_{i}-\mathbf{e}_{j}, \quad i<j
$$

are positive and the roots

$$
\alpha_{i} \equiv \alpha_{i, i+1}=\mathbf{e}_{i}-\mathbf{e}_{i+1}, \quad i=1,2, \ldots, n,
$$

constitute the system of simple roots.

If $x=\sum_{i=1}^{n+1} x_{i} \mathbf{e}_{i}, x_{1}+x_{2}+\cdots+x_{n+1}=0$, is a point of $E_{n+1}$, then this point belongs to the dominant Weyl chamber $D_{+}$if and only if

$$
x_{1} \geq x_{2} \geq \cdots \geq x_{n+1}
$$


Indeed, if this condition is fulfilled, then $\left\langle x, \alpha_{i}\right\rangle=x_{i}-x_{i+1} \geq 0, i=1,2, \ldots, n$, and $x$ is dominant. Conversely, if $x$ is dominant, then $\left\langle x, \alpha_{i}\right\rangle \geq 0$ and this condition is fulfilled. The point $x$ is strictly dominant if and only if

$$
x_{1}>x_{2}>\cdots>x_{n+1} .
$$

If $\lambda=\sum_{i=1}^{n} \lambda_{i} \omega_{i}$, then the coordinates $\lambda_{i}$ in the $\omega$-coordinates are connected with the orthogonal coordinates $m_{j}$ of $\lambda=\sum_{i=1}^{n+1} m_{i} \mathbf{e}_{i}$ by the formulas

$$
\begin{aligned}
& m_{1}=\frac{n}{n+1} \lambda_{1}+\frac{n-1}{n+1} \lambda_{2}+\frac{n-2}{n+1} \lambda_{3}+\cdots+\frac{2}{n+1} \lambda_{n-1}+\frac{1}{n+1} \lambda_{n}, \\
& m_{2}=-\frac{1}{n+1} \lambda_{1}+\frac{n-1}{n+1} \lambda_{2}+\frac{n-2}{n+1} \lambda_{3}+\cdots+\frac{2}{n+1} \lambda_{n-1}+\frac{1}{n+1} \lambda_{n}, \\
& m_{3}=-\frac{1}{n+1} \lambda_{1}-\frac{2}{n+1} \lambda_{2}+\frac{n-2}{n+1} \lambda_{3}+\cdots+\frac{2}{n+1} \lambda_{n-1}+\frac{1}{n+1} \lambda_{n}, \\
& m_{n}=-\frac{1}{n+1} \lambda_{1}-\frac{2}{n+1} \lambda_{2}-\frac{3}{n+1} \lambda_{3}-\cdots-\frac{n-1}{n+1} \lambda_{n-1}+\frac{1}{n+1} \lambda_{n}, \\
& m_{n+1}=-\frac{1}{n+1} \lambda_{1}-\frac{2}{n+1} \lambda_{2}-\frac{3}{n+1} \lambda_{3}-\cdots-\frac{n-1}{n+1} \lambda_{n-1}-\frac{n}{n+1} \lambda_{n} .
\end{aligned}
$$

The inverse formulas are

$$
\lambda_{i}=m_{i}-m_{i+1}, \quad i=1,2, \ldots, n .
$$

By means of the formula

$$
r_{\alpha} \lambda=\lambda-\frac{2\langle\lambda, \alpha\rangle}{\langle\alpha, \alpha\rangle} \alpha
$$

for the reflection with respect to the hyperplane, orthogonal to a root $\alpha$, we can find that the reflection $r_{\alpha_{i j}}$ acts upon the vector $\lambda=\sum_{i=1}^{n+1} m_{i} \mathbf{e}_{i}$, given by orthogonal coordinates, by permuting the coordinates $m_{i}$ and $m_{j}$. Since for each $i$ and $j, 1 \leq i, j \leq n+1$, there exists a root $\alpha_{i j}$, the Weyl group $W\left(A_{n}\right)$ consists of all permutations of the orthogonal coordinates $m_{1}, m_{2}, \ldots, m_{n+1}$ of a point $\lambda$, that is, $W\left(A_{n}\right)$ coincides with the symmetric group $S_{n+1}$.

Sometimes (for example, if we wish that coordinates would be integers or non-negative integers), it is convenient to introduce orthogonal coordinates $x_{1}, x_{2}, \ldots, x_{n+1}$ for $A_{n}$ in such a way that

$$
x_{1}+x_{2}+\cdots+x_{n+1}=m,
$$

where $m$ is some fixed real number. They are obtained from the previous orthogonal coordinates by adding the same number $m /(n+1)$ to each coordinate. Then, as one can see from (3.6), $\omega$-coordinates $\lambda_{i}=x_{i}-x_{i+1}$ and the Weyl group $W$ do not change. Sometimes, it is natural to use orthogonal coordinates $x_{1}, x_{2}, \ldots, x_{n+1}$ for which all $x_{i}$ are non-negative.

We need below the half-sum $\rho$ of the positive roots of $A_{n}, \rho=\frac{1}{2} \sum_{\alpha>0} \alpha$. It is easy to see that up to a common constant we have

$$
\rho=n \mathbf{e}_{1}+(n-1) \mathbf{e}_{2}+\cdots+\mathbf{e}_{n},
$$


that is, in orthogonal coordinates we have

$$
\rho=(n, n-1, \ldots, 1,0) .
$$

In the non-orthogonal $\omega$-coordinates we have

$$
\rho=\omega_{1}+\omega_{2}+\cdots+\omega_{n} .
$$

The signed orbit $O^{ \pm}(\lambda), \lambda=\left(m_{1}, m_{2}, \ldots, m_{n+1}\right), m_{1}>m_{2}>\cdots>m_{n+1}$, consists of all points

$$
\left(m_{i_{1}}, m_{i_{2}}, \ldots, m_{i_{n+1}}\right)^{\operatorname{sgn}(\operatorname{det} w)}
$$

obtained from $\left(m_{1}, m_{2}, \ldots, m_{n+1}\right)$ by permutations $w \in W \equiv S_{n+1}$. Below instead of $\operatorname{sgn}(\operatorname{det} w)$ we write simply $\operatorname{det} w$.

\subsection{The case of $B_{n}$}

Orthogonal coordinates of a point $x \in E_{n}$ are denoted by $x_{1}, x_{2}, \ldots, x_{n}$. We denote by $\mathbf{e}_{i}$ the corresponding unit vectors. Then the set of roots of $B_{n}$ is given by the vectors

$$
\alpha_{ \pm i, \pm j}= \pm \mathbf{e}_{i} \pm \mathbf{e}_{j}, \quad i \neq j, \quad \alpha_{ \pm i}= \pm \mathbf{e}_{i}, \quad i=1,2, \ldots, n
$$

(all combinations of signs must be taken). The roots

$$
\alpha_{i, \pm j}=\mathbf{e}_{i} \pm \mathbf{e}_{j}, \quad i<j, \quad \alpha_{i}=\mathbf{e}_{i}, \quad i=1,2, \ldots, n
$$

are positive and $n$ roots

$$
\alpha_{i}:=\mathbf{e}_{i}-\mathbf{e}_{i+1}, \quad i=1,2, \ldots, n-1, \quad \alpha_{n}=\mathbf{e}_{n}
$$

constitute the system of simple roots.

It is easy to see that if $\lambda=\sum_{i=1}^{n} m_{i} \mathbf{e}_{i}$ is a point of $E_{n}$, then this point belongs to the dominant Weyl chamber $D_{+}$if and only if

$$
m_{1} \geq m_{2} \geq \cdots \geq m_{n} \geq 0 .
$$

Moreover, this point is strictly dominant if and only if

$$
m_{1}>m_{2}>\cdots>m_{n}>0 \text {. }
$$

If $\lambda=\sum_{i=1}^{n} \lambda_{i} \omega_{i}$, then the coordinates $\lambda_{i}$ in the $\omega$-coordinates are connected with the coordinates $m_{j}$ of $\lambda=\sum_{i=1}^{n} m_{i} \mathbf{e}_{i}$ by the formulas

$$
\begin{aligned}
& m_{1}=\lambda_{1}+\lambda_{2}+\cdots+\lambda_{n-1}+\frac{1}{2} \lambda_{n}, \\
& m_{2}=\lambda_{2}+\cdots+\lambda_{n-1}+\frac{1}{2} \lambda_{n}, \\
& \begin{array}{lllllll} 
& \ldots & \ldots & \ldots & \ldots & \ldots & \ldots
\end{array} \\
& m_{n}=\quad \frac{1}{2} \lambda_{n},
\end{aligned}
$$

The inverse formulas are

$$
\lambda_{i}=m_{i}-m_{i+1}, \quad i=1,2, \ldots, n-1, \quad \lambda_{n}=2 m_{n} .
$$


It is easy to see that if $\lambda \in P_{+}$, then the coordinates $m_{1}, m_{1}, \ldots, m_{n}$ are all integers or all half-integers.

The half-sum $\rho$ of positive roots of $B_{n}, \rho=\frac{1}{2} \sum_{\alpha>0} \alpha$, in orthogonal coordinates has the form

$$
\rho=\left(n-\frac{1}{2}, n-\frac{3}{2}, \ldots, \frac{1}{2}\right)
$$

In $\omega$-coordinates we have $\rho=\omega_{1}+\omega_{2}+\cdots+\omega_{n}$.

By means of the formula (3.7) we find that the reflection $r_{\alpha}$ acts upon orthogonal coordinates of the vector $\lambda=\sum_{i=1}^{n} m_{i} \mathbf{e}_{i}$ by permuting $i$-th and $j$-th coordinates if $\alpha= \pm\left(\mathbf{e}_{i}-\mathbf{e}_{j}\right)$, as the permutation of $i$-th and $j$-th coordinates and the change of their signs if $\alpha= \pm\left(\mathbf{e}_{i}+\mathbf{e}_{j}\right)$, and as the change of a sign of $i$-th coordinate if $\alpha= \pm \mathbf{e}_{i}$. Thus, the Weyl group $W\left(B_{n}\right)$ consists of all permutations of the orthogonal coordinates $m_{1}, m_{2}, \ldots, m_{n}$ of a point $\lambda$ with possible sign alternations of any number of them.

The signed orbit $O^{ \pm}(\lambda), \lambda=\left(m_{1}, m_{2}, \ldots, m_{n}\right), m_{1}>m_{2}>\cdots>m_{n}>0$, consists of all points

$$
\left( \pm m_{i_{1}}, \pm m_{i_{2}}, \ldots, \pm m_{i_{n}}\right)^{\operatorname{det} w}
$$

(each combination of signs is possible) obtained from $\left(m_{1}, m_{2}, \ldots, m_{n}\right)$ by permutations and alternations of signs which constitute an element $w$ of the Weyl group $W\left(B_{n}\right)$. Moreover, $\operatorname{det} w$ is equal to \pm 1 depending on whether $w$ consists of even or odd number of reflections and alternations of signs. A sign of $\operatorname{det} w$ can be determined as follows. We represent $w$ as a product $w=\epsilon s$, where $s$ is a permutation of $\left(m_{1}, m_{2}, \ldots, m_{n}\right)$ and $\epsilon$ is an alternation of signs of coordinates. Then $\operatorname{det} w=(\operatorname{det} s) \epsilon_{i_{1}} \epsilon_{i_{2}} \cdots \epsilon_{i_{n}}$, where $\operatorname{det} s$ is defined as in the previous subsection and $\epsilon_{i_{j}}$ is a sign of $i_{j}$-th coordinate.

\subsection{The case of $C_{n}$}

In the orthogonal system of coordinates of the Euclidean space $E_{n}$ the set of roots of $C_{n}$ is given by the vectors

$$
\alpha_{ \pm i, \pm j}= \pm \mathbf{e}_{i} \pm \mathbf{e}_{j}, \quad i \neq j, \quad \alpha_{ \pm i}= \pm 2 \mathbf{e}_{i}, \quad i=1,2, \ldots, n
$$

where $\mathbf{e}_{i}$ is the unit vector in the direction of $i$-th coordinate $x_{i}$ (all combinations of signs must be taken). The roots

$$
\alpha_{i, \pm j}=\mathbf{e}_{i} \pm \mathbf{e}_{j}, \quad i<j, \quad \alpha_{i}=2 \mathbf{e}_{i}, \quad i=1,2, \ldots, n
$$

are positive and $n$ roots

$$
\alpha_{i}:=\mathbf{e}_{i}-\mathbf{e}_{i+1}, \quad i=1,2, \ldots, n-1, \quad \alpha_{n}=2 \mathbf{e}_{n}
$$

constitute the system of simple roots.

It is easy to see that a point $\lambda=\sum_{i=1}^{n} m_{i} \mathbf{e}_{i} \in E_{n}$ belongs to the dominant Weyl chamber $D_{+}$ if and only if

$$
m_{1} \geq m_{2} \geq \cdots \geq m_{n} \geq 0
$$

This point is strictly dominant if and only if

$$
m_{1}>m_{2}>\cdots>m_{n}>0 \text {. }
$$


If $\lambda=\sum_{i=1}^{n} \lambda_{i} \omega_{i}$, then the coordinates $\lambda_{i}$ in the $\omega$-coordinates are connected with the coordinates $m_{j}$ of $\lambda=\sum_{i=1}^{n} m_{i} \mathbf{e}_{i}$ by the formulas

$$
\begin{aligned}
& m_{1}=\lambda_{1}+\lambda_{2}+\cdots+\lambda_{n-1}+\lambda_{n}, \\
& m_{2}=\lambda_{2}+\cdots+\lambda_{n-1}+\lambda_{n}, \\
& \begin{array}{llllll}
\ldots & \ldots & \ldots & \ldots & \ldots & \ldots
\end{array} \\
& m_{n}=\quad \lambda_{n} .
\end{aligned}
$$

The inverse formulas are

$$
\lambda_{i}=m_{i}-m_{i+1}, \quad i=1,2, \ldots, n-1, \quad \lambda_{n}=m_{n} .
$$

If $\lambda \in P_{+}$, then all coordinates $m_{i}$ are integers.

The half-sum $\rho$ of positive roots of $C_{n}, \rho=\frac{1}{2} \sum_{\alpha>0} \alpha$, in orthogonal coordinates has the form

$$
\rho=(n, n-1, \ldots, 2,1)
$$

By means of the formula (3.7) we find that the reflection $r_{\alpha}$ acts upon orthogonal coordinates of the vector $\lambda=\sum_{i=1}^{n} m_{i} \mathbf{e}_{i}$ by permuting $i$-th and $j$-th coordinates if $\alpha= \pm\left(\mathbf{e}_{i}-\mathbf{e}_{j}\right)$, as the permutation of $i$-th and $j$-th coordinates and the change of their signs if $\alpha= \pm\left(\mathbf{e}_{i}+\mathbf{e}_{j}\right)$, and as the change of a sign of $i$-th coordinate if $\alpha= \pm 2 \mathbf{e}_{i}$. Thus, the Weyl group $W\left(C_{n}\right)$ consists of all permutations of the orthogonal coordinates $m_{1}, m_{2}, \ldots, m_{n}$ of a point $\lambda$ with sign alternations of some of them, that is, this Weyl group acts on orthogonal coordinates exactly in the same way as the Weyl group $W\left(B_{n}\right)$ does.

The signed orbit $O^{ \pm}(\lambda), \lambda=\left(m_{1}, m_{2}, \ldots, m_{n}\right), m_{1}>m_{2}>\cdots>m_{n}>0$, consists of all points

$$
\left( \pm m_{i_{1}}, \pm m_{i_{2}}, \ldots, \pm m_{i_{n+1}}\right)^{\operatorname{det} w}
$$

(each combination of signs is possible) obtained from $\left(m_{1}, m_{2}, \ldots, m_{n}\right)$ by permutations and alternations of signs which constitute an element $w$ of the Weyl group $W\left(C_{n}\right)$. Moreover, det $w$ is equal to \pm 1 depending on whether $w$ consists of even or odd numbers of reflections and alternations of signs. Since $W\left(C_{n}\right)=W\left(B_{n}\right)$, then a sign of $\operatorname{det} w$ is determined as in the case $B_{n}$.

As we see, in the orthogonal coordinates signed orbits for $C_{n}$ coincide with signed orbits of $B_{n}$.

\subsection{The case of $D_{n}$}

In the orthogonal system of coordinates of the Euclidean space $E_{n}$ the set of roots of $D_{n}$ is given by the vectors

$$
\alpha_{ \pm i, \pm j}= \pm \mathbf{e}_{i} \pm \mathbf{e}_{j}, \quad i \neq j
$$

where $\mathbf{e}_{i}$ is the unit vector in the direction of $i$-th coordinate (all combinations of signs must be taken). The roots

$$
\alpha_{i, \pm j}=\mathbf{e}_{i} \pm \mathbf{e}_{j}, \quad i<j,
$$


are positive and $n$ roots

$$
\alpha_{i}:=\mathbf{e}_{i}-\mathbf{e}_{i+1}, \quad i=1,2, \ldots, n-1, \quad \alpha_{n}=\mathbf{e}_{n-1}+\mathbf{e}_{n}
$$

constitute the system of simple roots.

It is easy to see that if $\lambda=\sum_{i=1}^{n} m_{i} \mathbf{e}_{i}$ is a point of $E_{n}$, then this point belongs to the dominant Weyl chamber $D_{+}$if and only if

$$
m_{1} \geq m_{2} \geq \cdots \geq m_{n-1} \geq\left|m_{n}\right| .
$$

This point is strictly dominant if and only if

$$
m_{1}>m_{2}>\cdots>m_{n-1}>\left|m_{n}\right|
$$

(in particular, $m_{n}$ can take the value 0 ).

If $\lambda=\sum_{i=1}^{n} \lambda_{i} \omega_{i}$, then the coordinates $\lambda_{i}$ in the $\omega$-coordinates are connected with the coordinates $m_{j}$ of $\lambda=\sum_{i=1}^{n} m_{i} \mathbf{e}_{i}$ by the formulas

$$
\begin{aligned}
& m_{1}=\lambda_{1}+\lambda_{2}+\cdots+\lambda_{n-2}+\frac{1}{2}\left(\lambda_{n-1}+\lambda_{n}\right) \text {, } \\
& m_{2}=\lambda_{2}+\cdots+\lambda_{n-2}+\frac{1}{2}\left(\lambda_{n-1}+\lambda_{n}\right) \text {, } \\
& \begin{array}{llllllll}
\ldots & \ldots & \ldots & \ldots & \ldots & \ldots & \ldots
\end{array} \\
& m_{n-1}=\quad \frac{1}{2}\left(\lambda_{n-1}+\lambda_{n}\right), \\
& m_{n}=\quad \frac{1}{2}\left(\lambda_{n-1}-\lambda_{n}\right),
\end{aligned}
$$

The inverse formulas are

$$
\lambda_{i}=m_{i}-m_{i+1}, \quad i=1,2, \ldots, n-2, \quad \lambda_{n-1}=m_{n-1}+m_{n}, \quad \lambda_{n}=m_{n-1}-m_{n} .
$$

If $\lambda \in P_{+}$, then the coordinates $m_{1}, m_{2}, \ldots, m_{n}$ are all integers or all half-integers.

The half-sum $\rho$ of positive roots of $D_{n}, \rho=\frac{1}{2} \sum_{\alpha>0} \alpha$, in orthogonal coordinates has the form

$$
\rho=(n-1, n-2, \ldots, 1,0) .
$$

By means of the formula (3.7) for the reflection $r_{\alpha}$ we find that $r_{\alpha}$ acts upon orthogonal coordinates of the vector $\lambda=\sum_{i=1}^{n} m_{i} \mathbf{e}_{i}$ by permuting $i$-th and $j$-th coordinates if $\alpha= \pm\left(\mathbf{e}_{i}-\mathbf{e}_{j}\right)$, and as the permutation of $i$-th and $j$-th coordinates and the change of their signs if $\alpha= \pm\left(\mathbf{e}_{i}+\right.$ $\left.\mathbf{e}_{j}\right)$. Thus, the Weyl group $W\left(D_{n}\right)$ consists of all permutations of the orthogonal coordinates $m_{1}, m_{2}, \ldots, m_{n}$ of a point $\lambda$ with sign alternations of even number of them.

Since an alternation of signs of two coordinates $x_{i}$ and $x_{j}$ is a product of two reflections $r_{\alpha}$ with $\alpha=\left(\mathbf{e}_{i}+\mathbf{e}_{j}\right)$ and with $\alpha=\left(\mathbf{e}_{i}-\mathbf{e}_{j}\right)$, a sign of the determinant of this alternation is plus. Note that $\left|W\left(D_{n}\right)\right|=\frac{1}{2}\left|W\left(B_{n}\right)\right|$.

The signed orbit $O^{ \pm}(\lambda), \lambda=\left(m_{1}, m_{2}, \ldots, m_{n}\right), m_{1}>m_{2}>\cdots>m_{n}>0$, consists of all points

$$
\left( \pm m_{i_{1}}, \pm m_{i_{2}}, \ldots, \pm m_{i_{n+1}}\right)^{\operatorname{det} w}
$$

obtained from $\left(m_{1}, m_{2}, \ldots, m_{n}\right)$ by permutations and alternations of even number of signs which constitute an element $w$ of the Weyl group $W\left(D_{n}\right)$. Moreover, $\operatorname{det} w$ is equal to \pm 1 and a sign of $\operatorname{det} w$ is determined as follows. The element $w \in W\left(D_{n}\right)$ can be represented as a product $w=\tau s$, where $s$ is a permutation from $S_{n}$ and $\tau$ is an alternation of even number of coordinates. Then $\operatorname{det} w=\operatorname{det} s$. Indeed, a determinant of a transform, given by an element of $W$ which is an alternation of two signs, is equal to +1 (since this element can be represented as a product of two reflections). 


\subsection{Signed orbits of $A_{3}$}

Signed orbits for $A_{3}, B_{3}$ and $C_{3}$ can be calculated by using the orthogonal coordinates in the corresponding Euclidean space, described above, and the description of the Weyl groups $W\left(A_{3}\right), W\left(B_{3}\right)$ and $W\left(C_{3}\right)$ in the orthogonal coordinate systems. Below we give results of such calculations. Points $\lambda$ of signed orbits are given in the $\omega$-coordinates as $(a b c)$, where $\lambda=a \omega_{1}+b \omega_{2}+c \omega_{3}$.

The signed orbit $O^{ \pm}(a b c), a>0, b>0, c>0$, of $A_{3}$ contains the points

$$
\begin{aligned}
O^{ \pm}(a b c) & \ni(a b c)^{+},(a+b-b b+c)^{-},(a+b c-b-c)^{+},(a b+c-c)^{-}, \\
& (a+b+c-c-b)^{-},(a+b+c-b-c b)^{+},(-a a+b c)^{-},(-a a+b+c-c)^{+}, \\
& (b-a-b a+b+c)^{+},(b+c-a-b-c a+b)^{-},(-a-b a b+c)^{+},(-b-a a+b+c)^{-}
\end{aligned}
$$

and the points, contragredient to these points, where the contragredient of the point $\left(a^{\prime} b^{\prime} c^{\prime}\right)^{+}$ is $\left(-c^{\prime}-b^{\prime}-a^{\prime}\right)^{+}$and the contragredient of the point $\left(a^{\prime} b^{\prime} c^{\prime}\right)^{-}$is $\left(-c^{\prime}-b^{\prime}-a^{\prime}\right)^{-}$.

\subsection{Signed orbits of $B_{3}$}

As in the previous case, points $\lambda$ of signed orbits are given by the $\omega$-coordinates $(a b c)$, where $\lambda=a \omega_{1}+b \omega_{2}+c \omega_{3}$. The signed orbit $O^{ \pm}(a b c), a>0, b>0, c>0$, of $B_{3}$ contains the points

$$
\begin{aligned}
O^{ \pm}(a b c) & \ni(a b c)^{+},(a+b-b 2 b+c)^{-},(-a a+b c)^{-},(b-a-b 2 a+2 b+c)^{+}, \\
& (-a-b a 2 b+c)^{+},(-b-a 2 a+2 b+c)^{-},(a b+c-c)^{-},(a+b+c-b-c 2 b+c)^{+}, \\
& (-a a+b+c-c)^{+},(b+c-a-b-c 2 a+2 b+c)^{-},(-a-b-c a 2 b+c)^{-}, \\
& (-b-c-a 2 a+2 b+c)^{+},(-a-2 b-c b c)^{-},(-a-b-c-b 2 b+c)^{+}, \\
& (a+2 b+c-a-b-c c)^{+},(b a+b+c-2 a-2 b-c)^{-},(a+b+c-a-2 b-c 2 b+c)^{-}, \\
& (-b a+2 b+c-2 a-2 b-c)^{+},(-a-2 b-c b+c-c)^{+},(-a-b-b-c 2 b+c)^{-}, \\
& (a+2 b+c-a-b-c)^{-},(b+c a+b-2 a-2 b-c)^{+},(a+b-a-2 b-c 2 b+c)^{+}, \\
& (-b-c a+2 b+c-2 a-2 b-c)^{-}
\end{aligned}
$$

and also all these points taken with opposite signs of coordinates, signs of these points are also opposite.

\subsection{Signed orbits of $C_{3}$}

As in the previous cases, points $\lambda$ of signed orbits are given by the $\omega$-coordinates $(a b c)$, where $\lambda=a \omega_{1}+b \omega_{2}+c \omega_{3}$. The signed orbit $O^{ \pm}(a b c), a>0, b>0, c>0$, of $C_{3}$ contains the points

$$
\begin{aligned}
O^{ \pm}(a b c) & \ni(a b c)^{+},(a+b-b b+c)^{-},(-a a+b c)^{-},(b-a-b a+b+c)^{+}, \\
& (-a-b a b+c)^{+},(-b-a a+b+c)^{-},(a b+2 c-c)^{-},(a+b+2 c-b-2 c b+c)^{+}, \\
& (-a a+b+2 c-c)^{+},(b+2 c-a-b-2 c a+b+c)^{-},(-a-b-2 c a b+c)^{-}, \\
& (-b-2 c-a a+b+c)^{+},(-a-2 b-2 c b c)^{-},(-a-b-2 c-b b+c)^{+}, \\
& (a+2 b+2 c-a-b-2 c c)^{+},(b a+b+2 c-a-b-c)^{-},(a+b+2 c-a-2 b-2 c b+c)^{-}, \\
& (-b a+2 b+2 c-a-b-c)^{+},(-a-2 b-2 c b+2 c-c)^{+},(-a-b-b-2 c b+c)^{-}, \\
& (a+2 b+2 c-a-b-c)^{-},(b+2 c a+b-a-b-c)^{+},(a+b-a-2 b-2 c b+c)^{+}, \\
& (-b-2 c a+2 b+2 c-a-b-c)^{-}
\end{aligned}
$$

and also all these points taken with opposite signs of coordinates, signs of these points are also opposite. 


\section{Antisymmetric orbit functions}

\subsection{Definition}

The exponential functions $e^{2 \pi \mathrm{i}\langle m, x\rangle}, x \in E_{n}$, with fixed $m=\left(m_{1}, m_{2}, \ldots, m_{n}\right)$ determine the Fourier transform on $E_{n}$. Antisymmetric orbit functions are an antisymmetrized (with respect to a Weyl group) version of exponential functions. Correspondingly, they determine an antisymmetrized version of the Fourier transform.

First we define symmetric orbit functions, studied in [1]. Let $W$ be a Weyl group of transformations of the Euclidean space $E_{n}$. To each element $\lambda \in E_{n}$ from the dominant Weyl chamber (that is, $\left\langle\lambda, \alpha_{i}\right\rangle \geq 0$ for all simple roots $\alpha_{i}$ ) there corresponds a symmetric orbit function $\phi_{\lambda}$ on $E_{n}$, which is given by the formula

$$
\phi_{\lambda}(x)=\sum_{\mu \in O(\lambda)} e^{2 \pi \mathrm{i}\langle\mu, x\rangle}, \quad x \in E_{n}
$$

where $O(\lambda)$ is the $W$-orbit of the element $\lambda$. The number of summands is equal to the size $|O(\lambda)|$ of the orbit $O(\lambda)$ and we have $\phi_{\lambda}(0)=|O(\lambda)|$.

Sometimes (see, for example, [14] and [15]), it is convenient to use a modified definition of orbit functions:

$$
\hat{\phi}_{\lambda}(x)=\left|W_{\lambda}\right| \phi_{\lambda}(x)
$$

where $W_{\lambda}$ is a subgroup in $W$ whose elements leave $\lambda$ fixed. Then for all orbit functions $\hat{\phi}_{\lambda}$ we have $\hat{\phi}_{\lambda}(0)=|W|$.

Antisymmetric orbit functions are defined (see [22] and [29]) for dominant elements $\lambda$, which do not belong to a wall of the dominant Weyl chamber (that is, for strictly dominant elements $\lambda$ ). The antisymmetric orbit function, corresponding to such an element, is defined as

$$
\varphi_{\lambda}(x)=\sum_{w \in W}(\operatorname{det} w) e^{2 \pi \mathrm{i}\langle w \lambda, x\rangle}, \quad x \in E_{n}
$$

A number of summands in (4.3) is equal to the size $|W|$ of the Weyl group $W$. We have $\varphi_{\lambda}(0)=0$.

Symmetric orbit functions $\phi_{\lambda}$ for which $\lambda \in P_{+}$and antisymmetric orbit functions $\varphi_{\lambda}(x)$ for which $\lambda \in P_{+}^{+}$are of special interest for representation theory.

Example. Antisymmetric orbit functions for $A_{1}$. In this case, there exists only one simple (positive) root $\alpha$. We have $\langle\alpha, \alpha\rangle=2$. Then the relation $2\langle\omega, \alpha\rangle /\langle\alpha, \alpha\rangle=1$ means that $\langle\omega, \alpha\rangle=1$. This means that $\omega=\alpha / 2$ and $\langle\omega, \omega\rangle=1 / 2$. Elements of $P_{+}^{+}$coincide with $m \omega$, $m \in \mathbb{Z}_{+}$. We identify points $x$ of $E_{1} \equiv \mathbb{R}$ with $\theta \omega$. Since the Weyl group $W\left(A_{1}\right)$ consists of two elements 1 and $r_{\alpha}$, and

$$
r_{\alpha} x=x-\frac{2\langle\theta \omega, \alpha\rangle}{\langle\alpha, \alpha\rangle} \alpha=x-\theta \alpha=x-2 x=-x,
$$

antisymmetric orbit functions $\varphi_{\lambda}(x), \lambda=m \omega, m>0$, are given by the formula

$$
\varphi_{\lambda}(x)=e^{2 \pi \mathrm{i}\langle m \omega, \theta \omega\rangle}-e^{-2 \pi \mathrm{i}\langle m \omega, \theta \omega\rangle}=e^{\pi \mathrm{i} m \theta}-e^{-\pi \mathrm{i} m \theta}=2 \mathrm{i} \sin (\pi m \theta) .
$$

Note that for the symmetric orbit function $\phi_{\lambda}(x)$ we have $\phi_{\lambda}(x)=2 \cos (\pi m \theta)$. 


\subsection{Antisymmetric orbit functions of $\boldsymbol{A}_{2}$}

Antisymmetric orbit functions for the Coxeter-Dynkin diagrams of rank 2 were given in [22]. In this subsection we give these functions for $A_{2}$. that

Put $\lambda=a \omega_{1}+b \omega_{2} \equiv(a b)$ with $a>0, b>0$. Then for $\varphi_{\lambda}(x) \equiv \varphi_{(a b)}(x)$ we have from (4.3)

$$
\begin{aligned}
\varphi_{(a b)}(x)= & e^{2 \pi i\langle(a b), x\rangle}-e^{2 \pi i\langle(-a a+b), x\rangle}-e^{2 \pi i\langle(a+b-b), x\rangle} \\
& +e^{2 \pi i\langle(b-a-b), x\rangle}+e^{2 \pi i\langle(-a-b a), x\rangle}-e^{2 \pi i\langle(-b-a), x\rangle} .
\end{aligned}
$$

Using the representation $x=\psi_{1} \alpha_{1}+\psi_{2} \alpha_{2}$, one obtains

$$
\begin{aligned}
\varphi_{(a b)}(x)= & e^{2 \pi i\left(a \psi_{1}+b \psi_{2}\right)}-e^{2 \pi i\left(-a \psi_{1}+(a+b) \psi_{2}\right)}-e^{2 \pi i\left((a+b) \psi_{1}-b \psi_{2}\right)} \\
& +e^{2 \pi i\left(b \psi_{1}-(a+b) \psi_{2}\right)}+e^{2 \pi i\left((-a-b) \psi_{1}+a \psi_{2}\right)}-e^{2 \pi i\left(-b \psi_{1}-a \psi_{2}\right)} .
\end{aligned}
$$

The actual expression for $\varphi_{(a b)}(x)$ depends on a choice of coordinate systems for $\lambda$ and $x$. Setting $x=\theta_{1} \omega_{1}+\theta_{2} \omega_{2}$ and $\lambda$ as before, we get

$$
\begin{aligned}
\varphi_{(a b)}(x)= & e^{\frac{2 \pi i}{3}\left((2 a+b) \theta_{1}+(a+2 b) \theta_{2}\right)}-e^{\frac{2 \pi i}{3}\left((-a+b) \theta_{1}+(a+2 b) \theta_{2}\right)} \\
& -e^{\frac{2 \pi i}{3}\left((2 a+b) \theta_{1}+(a-b) \theta_{2}\right)}+e^{-\frac{2 \pi i}{3}\left((a-b) \theta_{1}+(2 a+b) \theta_{2}\right)} \\
& +e^{-\frac{2 \pi i}{3}\left((a+2 b) \theta_{1}+(-a+b) \theta_{2}\right)}-e^{-\frac{2 \pi i}{3}\left((a+2 b) \theta_{1}+(2 a+b) \theta_{2}\right)} .
\end{aligned}
$$

Note that $\left.\phi_{(a} a\right)(x)$ are pure imaginary for all $a>0$ and

$$
\begin{aligned}
\varphi_{(a \text { a })}(x) & =2 \mathrm{i}\left\{\sin 2 \pi a\left(\psi_{1}+\psi_{2}\right)+\sin 2 \pi a\left(\psi_{1}-2 \psi_{2}\right)-\sin 2 \pi a\left(2 \psi_{1}-\psi_{2}\right)\right\} \\
& =2 \mathrm{i}\left\{\sin 2 \pi a\left(\theta_{1}+\theta_{2}\right)-\sin 2 \pi a \theta_{1}-\sin 2 \pi a \theta_{2}\right\} .
\end{aligned}
$$

The pairs $\varphi_{(a b)}(x)+\varphi_{(b a)}(x)$ are always pure imaginary functions.

\subsection{Antisymmetric orbit functions of $C_{2}$ and $G_{2}$}

Putting again $\lambda=a \omega_{1}+b \omega_{2}=(a b), x=\theta_{1} \omega_{1}+\theta_{2} \omega_{2}$ and using the matrices $S$ from (2.6), which are of the form

$$
S\left(C_{2}\right)=\frac{1}{2}\left(\begin{array}{ll}
1 & 1 \\
1 & 2
\end{array}\right), \quad S\left(G_{2}\right)=\frac{1}{6}\left(\begin{array}{ll}
6 & 3 \\
3 & 2
\end{array}\right),
$$

we find the orbit functions for $C_{2}$ and $G_{2}$ :

$$
\begin{aligned}
C_{2}: \quad \varphi_{(a b)}(x)= & 2 \cos \pi\left((a+b) \theta_{1}+(a+2 b) \theta_{2}\right)-2 \cos \pi\left(b \theta_{1}+(a+2 b) \theta_{2}\right) \\
& -2 \cos \pi\left((a+b) \theta_{1}+a \theta_{2}\right)+2 \cos \pi\left(b \theta_{1}-a \theta_{2}\right), \\
G_{2}: \quad \varphi_{(a b)}(x)= & 2 \cos \pi\left((2 a+b) \theta_{1}+\left(a+\frac{2}{3} b\right) \theta_{2}\right)-2 \cos \pi\left((a+b) \theta_{1}+\left(a+\frac{2}{3} b\right) \theta_{2}\right) \\
& -2 \cos \pi\left((2 a+b) \theta_{1}+\left(a+\frac{1}{3} b\right) \theta_{2}\right)+2 \cos \pi\left((a+b) \theta_{1}+\frac{1}{3} b \theta_{2}\right) \\
& +2 \cos \pi\left(a \theta_{1}+\left(a+\frac{1}{3} b\right) \theta_{2}\right)-2 \cos \pi\left(a \theta_{1}-\frac{1}{3} b \theta_{2}\right) .
\end{aligned}
$$

As we see, orbit functions for $C_{2}$ and $G_{2}$ are real. 


\subsection{Antisymmetric orbit functions of $A_{n}$}

It is difficult to write down an explicit form of orbit functions for $A_{n}, B_{n}, C_{n}$ and $D_{n}$ in coordinates with respect to the $\omega$ - or $\alpha$-bases. For this reason, for these cases we use the orthogonal coordinate systems, described in Section 3.

Let $\lambda=\left(m_{1}, m_{2}, \ldots, m_{n+1}\right)$ be a strictly dominant element for $A_{n}$ in orthogonal coordinates described in Subsection 3.3. Then $m_{1}>m_{2}>\cdots>m_{n+1}$. The Weyl group in this case coincides with the symmetric group $S_{n+1}$. Then the signed orbit $O^{ \pm}(\lambda)$ consists of points $(w \lambda)^{\operatorname{det} w}, w \in W \equiv S_{n+1}$. Representing points $x \in E_{n+1}$ in the orthogonal coordinate system, $x=\left(x_{1}, x_{2}, \ldots, x_{n+1}\right)$, and using formula (4.3) we find that

$$
\begin{aligned}
\varphi_{\lambda}(x) & =\sum_{w \in S_{n+1}}(\operatorname{det} w) e^{2 \pi \mathrm{i}\left\langle w\left(m_{1}, \ldots, m_{n+1}\right),\left(x_{1}, \ldots, x_{n+1}\right)\right\rangle} \\
& =\sum_{w \in S_{n+1}}(\operatorname{det} w) e^{2 \pi \mathrm{i}\left((w \lambda)_{1} x_{1}+\cdots+(w \lambda)_{n+1} x_{n+1}\right)},
\end{aligned}
$$

where $\left((w \lambda)_{1},(w \lambda)_{2}, \ldots,(w \lambda)_{n+1}\right)$ are the coordinates of the point $w \lambda$.

Note that the element $-\left(m_{n+1}, m_{n}, \ldots, m_{1}\right)$ is strictly dominant if the element $\left(m_{1}, m_{2}, \ldots\right.$, $\left.m_{n+1}\right)$ is strictly dominant. In the Weyl group $W\left(A_{n}\right)$ there exists an element $w_{0}$ such that

$$
w_{0}\left(m_{1}, m_{2}, \ldots, m_{n+1}\right)=\left(m_{n+1}, m_{n}, \ldots, m_{1}\right) .
$$

Moreover, we have

$$
\begin{array}{lrrrrr}
\operatorname{det} w_{0} & =1 & \text { for } & A_{4 k-1} & \text { and } & A_{4 k}, \\
\operatorname{det} w_{0} & =-1 & \text { for } & A_{4 k+1} & \text { and } & A_{4 k+2} .
\end{array}
$$

It follows from here that in the expressions for the orbit functions $\varphi_{\left(m_{1}, m_{2}, \ldots, m_{n+1}\right)}(x)$ and $\varphi_{-\left(m_{n+1}, m_{n}, \cdots, m_{1}\right)}(x)$ there are summands

$$
e^{2 \pi \mathrm{i}\left\langle w_{0} \lambda, x\right\rangle}=e^{2 \pi \mathrm{i}\left(m_{n+1} x_{1}+\cdots+m_{1} x_{n+1}\right)} \quad \text { and } \quad e^{-2 \pi \mathrm{i}\left(m_{n+1} x_{1}+\cdots+m_{1} x_{n+1}\right)},
$$

respectively, which are complex conjugate to each other. Moreover, the first expression is contained with the sign $\left(\operatorname{det} w_{0}\right)$ in $\varphi_{\left(m_{1}, m_{2}, \ldots, m_{n+1}\right)}(x)$, that is, the expressions (4.10) are contained in $\varphi_{\left(m_{1}, m_{2}, \ldots, m_{n+1}\right)}(x)$ and $\varphi_{-\left(m_{n+1}, m_{n}, \ldots, m_{1}\right)}(x)$ with the same sign for $n=4 k-1,4 k$ and with opposite signs for $n=4 k+1,4 k+2, k \in \mathbb{Z}_{+}$.

Similarly, in the expressions (4.9) for the function $\varphi_{\left(m_{1}, m_{2}, \ldots, m_{n+1}\right)}(x)$ and for the function $\varphi_{-\left(m_{n+1}, m_{n}, \ldots, m_{1}\right)}(x)$ all other summands are (up to a sign, which depends on a value of $n$ ) pairwise complex conjugate. Therefore,

$$
\varphi_{\left(m_{1}, m_{2}, \ldots, m_{n+1}\right)}(x)=\overline{\varphi_{-\left(m_{n+1}, m_{n}, \ldots, m_{1}\right)}(x)}
$$

for $n=4 k-1,4 k$ and

$$
\varphi_{\left(m_{1}, m_{2}, \ldots, m_{n+1}\right)}(x)=-\overline{\varphi_{-\left(m_{n+1}, m_{n}, \ldots, m_{1}\right)}(x)}
$$

for $n=4 k+1,4 k+2$.

If we use for $\lambda$ the coordinates $\lambda_{i}=\left\langle\lambda, \alpha_{i}^{\vee}\right\rangle$ in the $\omega$-basis instead of the orthogonal coordinates $m_{j}$, then these equations can be written as

$$
\varphi_{\left(\lambda_{1}, \ldots, \lambda_{n}\right)}(x)=\overline{\varphi_{\left(\lambda_{n}, \ldots, \lambda_{1}\right)}(x)}, \quad \varphi_{\left(\lambda_{1}, \ldots, \lambda_{n}\right)}(x)=-\overline{\varphi_{\left(\lambda_{n}, \ldots, \lambda_{1}\right)}(x)},
$$

respectively. According to (4.11) and (4.12), if

$$
\left(m_{1}, m_{2}, \ldots, m_{n+1}\right)=-\left(m_{n+1}, m_{n}, \ldots, m_{1}\right)
$$


(that is, the element $\lambda$ has in the $\omega$-basis the coordinates $\left.\left(\lambda_{1}, \lambda_{2}, \ldots, \lambda_{2}, \lambda_{1}\right)\right)$, then the orbit function $\varphi_{\lambda}$ is real for $n=4 k-1,4 k$ and pure imaginary for $n=4 k+1,4 k+2$. In the first case the antisymmetric orbit function can be represented as a sum of cosines of angles and in the second case as a sum of sines of angles multiplied by $i=\sqrt{-1}$.

Proposition 2. In the orthogonal coordinates, antisymmetric orbit functions of $A_{n}$ can be represented as determinants of certain matrices:

$$
\begin{aligned}
\varphi_{\left(m_{1}, m_{2}, \ldots, m_{n+1}\right)}(x) & =\operatorname{det}\left(e^{2 \pi \mathrm{i} m_{i} x_{j}}\right)_{i, j=1}^{n+1} \\
& \equiv \operatorname{det}\left(\begin{array}{cccc}
e^{2 \pi \mathrm{i} m_{1} x_{1}} & e^{2 \pi \mathrm{i} m_{1} x_{2}} & \cdots & e^{2 \pi \mathrm{i} m_{1} x_{n+1}} \\
e^{2 \pi \mathrm{i} m_{2} x_{1}} & e^{2 \pi \mathrm{i} m_{2} x_{2}} & \cdots & e^{2 \pi \mathrm{i} m_{2} x_{n+1}} \\
\cdots & \cdots & \cdots & \cdots \\
e^{2 \pi \mathrm{i} m_{n+1} x_{1}} & e^{2 \pi \mathrm{i} m_{n+1} x_{2}} & \cdots & e^{2 \pi \mathrm{i} m_{n+1} x_{n+1}}
\end{array}\right) .
\end{aligned}
$$

Proof. A proof of this formula follows from the fact that this expression for $\varphi_{\left(m_{1}, m_{2}, \ldots, m_{n+1}\right)}(x)$ coincides with the expression given by the formula (4.9); see [30].

Taking into account the form of the half-sum of positive roots $\rho$ for $A_{n}$, we can write down the orbit function $\varphi_{\rho}(x)$, corresponding to the weight $\rho=\frac{1}{2} \sum_{\alpha>0} \alpha$, in the form of the Vandermonde determinant,

$$
\varphi_{\rho}(x)=\operatorname{det}\left(e^{2 \pi \mathrm{i} i x_{j}}\right)_{i, j=1}^{n+1}=\prod_{k<l}\left(e^{2 \pi \mathrm{i} x_{k}}-e^{-2 \pi \mathrm{i} x_{l}}\right) .
$$

The last equality follows from the expression for the Vandermonde determinant.

\subsection{Antisymmetric orbit functions of $B_{n}$}

Let $\lambda=\left(m_{1}, m_{2}, \ldots, m_{n}\right)$ be a strictly dominant element for $B_{n}$ in orthogonal coordinates described in Subsection 3.4. Then $m_{1}>m_{2}>\cdots>m_{n}>0$. The Weyl group $W\left(B_{n}\right)$ consists of permutations of the coordinates $m_{i}$ with sign alternations of some of them. Representing points $x \in E_{n}$ also in the orthogonal coordinate system, $x=\left(x_{1}, x_{2}, \ldots, x_{n}\right)$, and using formula (4.3) we find that

$$
\begin{aligned}
\varphi_{\lambda}(x) & =\sum_{\varepsilon_{i}= \pm 1} \sum_{w \in S_{n}}(\operatorname{det} w) \varepsilon_{1} \varepsilon_{2} \ldots \varepsilon_{n} e^{2 \pi \mathrm{i}\left\langle w\left(\varepsilon_{1} m_{1}, \ldots, \varepsilon_{n} m_{n}\right),\left(x_{1}, \ldots, x_{n}\right)\right\rangle} \\
& =\sum_{\varepsilon_{i}= \pm 1} \sum_{w \in S_{n}}(\operatorname{det} w) \varepsilon_{1} \varepsilon_{2} \cdots \varepsilon_{n} e^{2 \pi \mathrm{i}\left((w(\varepsilon \lambda))_{1} x_{1}+\cdots+(w(\varepsilon \lambda))_{n} x_{n}\right)}
\end{aligned}
$$

where $(w(\varepsilon \lambda))_{1}, \ldots,(w(\varepsilon \lambda))_{n}$ are the orthogonal coordinates of the points $w(\varepsilon \lambda)$ if $\varepsilon \lambda=\left(\varepsilon_{1} m_{1}\right.$, $\left.\ldots, \varepsilon_{n} m_{n}\right)$.

Since in $W\left(B_{n}\right)$ there exists an element which changes signs of all coordinates $m_{i}$, then for each summand $e^{2 \pi \mathrm{i}\left((w(\varepsilon \lambda))_{1} x_{1}+\cdots+(w(\varepsilon \lambda))_{n} x_{n}\right)}$ in the expressions (4.16) for the antisymmetric orbit function $\varphi_{\left(m_{1}, m_{2}, \ldots, m_{n}\right)}(x)$ there exists exactly one summand complex conjugate to it, that is, the summand $e^{-2 \pi \mathrm{i}\left(\left((w(\varepsilon \lambda))_{1} x_{1}+\cdots+(w(\varepsilon \lambda))_{n} x_{n}\right)\right.}$. This summand is with sign $(-1)^{n}=\left(\operatorname{det} w^{\prime}\right)$, where $w^{\prime}$ changes signs of all coordinates. Therefore, $\left(\operatorname{det} w^{\prime}\right)=1$ if $n=2 k$ and $\left(\operatorname{det} w^{\prime}\right)=-1$ if $n=2 k+1$. This means that antisymmetric orbit functions of $B_{n}$ are real if $n=2 k$ and pure imaginary if $n=2 k+1$. Each antisymmetric orbit function of $B_{n}$ can be represented as a sum of cosines of the corresponding angles if $n=2 k$ and as a sum of sines, multiplied by $\mathrm{i}=\sqrt{-1}$, if $n=2 k+1$. The following proposition is true [30]: 
Proposition 3. Antisymmetric orbit functions of $B_{n}$ can be represented in the form

$$
\varphi_{\left(m_{1}, m_{2}, \ldots, m_{n}\right)}(x)=\operatorname{det}\left(e^{2 \pi \mathrm{i} m_{i} x_{j}}-e^{-2 \pi \mathrm{i} m_{i} x_{j}}\right)_{i, j=1}^{n}=(2 \mathrm{i})^{n} \operatorname{det}\left(\sin 2 \pi m_{i} x_{j}\right)_{i, j=1}^{n} .
$$

Proof. Let us take on the right hand side of (4.16) the sum of terms with fixed $w \in S_{n}$. It can be written down as

$$
\begin{aligned}
(\operatorname{det} w) & \sum_{\varepsilon_{i}= \pm 1} \varepsilon_{1} \varepsilon_{2} \cdots \varepsilon_{n} e^{2 \pi \mathrm{i}\left((w(\varepsilon \lambda))_{1} x_{1}+\cdots+(w(\varepsilon \lambda))_{n} x_{n}\right)} \\
= & (\operatorname{det} w)\left(e^{2 \pi \mathrm{i}(w \lambda)_{1} x_{1}}-e^{-2 \pi \mathrm{i}(w \lambda)_{1} x_{1}}\right) \cdots\left(e^{2 \pi \mathrm{i}(w \lambda)_{n} x_{n}}-e^{-2 \pi \mathrm{i}(w \lambda)_{n} x_{n}}\right) \\
= & (\operatorname{det} w)(2 \mathrm{i})^{n} \sin 2 \pi(w \lambda)_{1} x_{1} \cdot \ldots \cdot \sin 2 \pi(w \lambda)_{n} x_{n} .
\end{aligned}
$$

Then for $\varphi_{\lambda}(x)$ we have

$$
\begin{aligned}
\varphi_{\lambda}(x) & =(2 \mathrm{i})^{n} \sum_{w \in S_{n}}(\operatorname{det} w) \sin 2 \pi(w \lambda)_{1} x_{1} \cdot \ldots \cdot \sin 2 \pi(w \lambda)_{n} x_{n} \\
& =(2 \mathrm{i})^{n} \operatorname{det}\left(\sin 2 \pi m_{i} x_{j}\right)_{i, j=1}^{n},
\end{aligned}
$$

where $\lambda=\left(m_{1}, m_{2}, \ldots, m_{n}\right)$. Proposition is proved.

For the antisymmetric orbit function $\varphi_{\rho}$, corresponding to the half-sum $\rho$ of positive roots of $B_{n}$, one has

$$
\varphi_{\rho}(x)=(2 \mathrm{i})^{n} \operatorname{det}\left(\sin 2 \pi \rho_{i} x_{j}\right)_{i, j=1}^{n},
$$

where $\rho=\left(\rho_{1}, \rho_{2}, \ldots, \rho_{n}\right)=\left(n-\frac{1}{2}, n-\frac{3}{2}, \ldots, \frac{1}{2}\right)$.

\subsection{Antisymmetric orbit functions of $C_{n}$}

Let $\lambda=\left(m_{1}, m_{2}, \ldots, m_{n}\right)$ be a strictly dominant element for $C_{n}$ in the orthogonal coordinates described in Subsection 3.5. Then $m_{1}>m_{2}>\cdots>m_{n}>0$. The Weyl group $W\left(C_{n}\right)$ consists of permutations of the coordinates with sign alternations of some of them. Representing points $x \in E_{n}$ also in the orthogonal coordinate system, $x=\left(x_{1}, x_{2}, \ldots, x_{n}\right)$, we find that

$$
\begin{aligned}
\varphi_{\lambda}(x) & =\sum_{\varepsilon_{i}= \pm 1} \sum_{w \in S_{n}}(\operatorname{det} w) \varepsilon_{1} \varepsilon_{2} \ldots \varepsilon_{n} e^{2 \pi \mathrm{i}\left\langle w\left(\varepsilon_{1} m_{1}, \ldots, \varepsilon_{n} m_{n}\right),\left(x_{1}, \cdots, x_{n}\right)\right\rangle} \\
& =\sum_{\varepsilon_{i}= \pm 1} \sum_{w \in S_{n}}(\operatorname{det} w) \varepsilon_{1} \varepsilon_{2} \cdots \varepsilon_{n} e^{2 \pi \mathrm{i}\left((w(\varepsilon \lambda))_{1} x_{1}+\cdots+(w(\varepsilon \lambda))_{n} x_{n}\right)},
\end{aligned}
$$

where, as above, $(w(\varepsilon \lambda))_{1}, \ldots,(w(\varepsilon \lambda))_{n}$ are the orthogonal coordinates of the points $w(\varepsilon \lambda)$ if $\varepsilon \lambda=\left(\varepsilon_{1} m_{1}, \ldots, \varepsilon_{n} m_{n}\right)$.

As in the case of $B_{n}$, in the expressions (4.18) for the functions $\varphi_{\left(m_{1}, m_{2}, \ldots, m_{n}\right)}(x)$ for each summand $e^{2 \pi \mathrm{i}\left((w(\varepsilon \lambda))_{1} x_{1}+\cdots+(w(\varepsilon \lambda))_{n} x_{n}\right)}$ there exists exactly one summand complex conjugate to it, that is, the summand $e^{-2 \pi \mathrm{i}\left((w(\varepsilon \lambda))_{1} x_{1}+\cdots+(w(\varepsilon \lambda))_{n} x_{n}\right)}$. Moreover, this summand is with sign "+" if $n=2 k$ and with sign "-" if $n=2 k+1$. Therefore, antisymmetric orbit functions of $C_{n}$ are real if $n=2 k$ and pure imaginary if $n=2 k+1$.

Note that in the orthogonal coordinates the antisymmetric orbit functions $\varphi_{\left(m_{1}, m_{2}, \ldots, m_{n}\right)}(x)$ of $C_{n}$ coincides with the antisymmetric orbit functions $\varphi_{\left(m_{1}, m_{2}, \ldots, m_{n}\right)}(x)$ of $B_{n}$, that is, antisymmetric orbit functions (4.16) and (4.18) coincide. However, $\alpha$-coordinates of the element $\left(m_{1}, m_{2}, \ldots, m_{n}\right)$ for $C_{n}$ do not coincide with $\alpha$-coordinates of the element $\left(m_{1}, m_{2}, \ldots, m_{n}\right)$ for $B_{n}$, that is, in $\alpha$-coordinates the corresponding antisymmetric orbit functions of $B_{n}$ and $C_{n}$ are different. 
Proposition 4. Antisymmetric orbit functions of $C_{n}$ can be represented in the form

$$
\varphi_{\left(m_{1}, m_{2}, \ldots, m_{n}\right)}(x)=\operatorname{det}\left(e^{2 \pi \mathrm{i} m_{i} x_{j}}-e^{-2 \pi \mathrm{i} m_{i} x_{j}}\right)_{i, j=1}^{n}=(2 \mathrm{i})^{n} \operatorname{det}\left(\sin 2 \pi m_{i} x_{j}\right)_{i, j=1}^{n} .
$$

Proof. This proposition follows from Proposition 3 if we take into account that antisymmetric orbit functions of $C_{n}$ and of $B_{n}$ coincide in the orthogonal coordinate systems.

For the antisymmetric orbit function $\varphi_{\rho}$, corresponding to the half-sum of positive roots of $C_{n}$, one has

$$
\varphi_{\rho}(x)=(2 \mathrm{i})^{n} \operatorname{det}\left(\sin 2 \pi \rho_{i} x_{j}\right)_{i, j=1}^{n},
$$

where $\rho=\left(\rho_{1}, \rho_{2}, \ldots, \rho_{n}\right)=(n, n-1, \ldots, 1)$.

\subsection{Antisymmetric orbit functions of $D_{n}$}

Let $\lambda=\left(m_{1}, m_{2}, \ldots, m_{n}\right)$ be a strictly dominant element for $D_{n}$ in the orthogonal coordinates described in Subsection 3.6. Then $m_{1}>m_{2}>\cdots>m_{n-1}>\left|m_{n}\right|$. The Weyl group $W\left(D_{n}\right)$ consists of permutations of the coordinates with sign alternations of even number of them. Representing points $x \in E_{n}$ also in the orthogonal coordinate system, $x=\left(x_{1}, x_{2}, \ldots, x_{n}\right)$, and using formula (4.3) we find that

$$
\begin{aligned}
\varphi_{\lambda}(x) & =\sum_{\varepsilon_{i}= \pm 1}^{\prime} \sum_{w \in S_{n}}(\operatorname{det} w) e^{2 \pi \mathrm{i}\left\langle w\left(\varepsilon_{1} m_{1}, \ldots, \varepsilon_{n} m_{n}\right),\left(x_{1}, \ldots, x_{n}\right)\right\rangle} \\
& =\sum_{\varepsilon_{i}= \pm 1}^{\prime} \sum_{w \in S_{n}}(\operatorname{det} w) e^{2 \pi \mathrm{i}\left((w(\varepsilon \lambda))_{1} x_{1}+\cdots+(w(\varepsilon \lambda))_{n} x_{n}\right)},
\end{aligned}
$$

where $(w(\varepsilon \lambda))_{1}, \ldots,(w(\varepsilon \lambda))_{n}$ are the orthogonal coordinates of the points $w(\varepsilon \lambda)$ and the prime at the sum sign means that the summation is over values of $\varepsilon_{i}$ with even number of sign minus. We have taken into account that an alternation of coordinates without any permutation does not change a determinant.

Let $m_{n} \neq 0$. Then in the expressions (4.20) for the orbit function $\varphi_{\left(m_{1}, m_{2}, \ldots, m_{n}\right)}(x)$ of $D_{n=2 k}$ for each summand $e^{2 \pi \mathrm{i}\left((w(\varepsilon \lambda))_{1} x_{1}+\cdots+(w(\varepsilon \lambda))_{n} x_{n}\right)}$ there exists exactly one summand (with the same sign) complex conjugate to it. This means that these antisymmetric orbit functions of $D_{2 k}$ are real. Each orbit function of $D_{2 k}$ can be represented as a sum of cosines of the corresponding angles.

It is also proved by using the formula (4.20) that for $m_{n} \neq 0$ the antisymmetric orbit functions $\varphi_{\left(m_{1}, \ldots, m_{2 k}, m_{2 k+1}\right)}(x)$ and $\varphi_{\left(m_{1}, \ldots, m_{2 k},-m_{2 k+1}\right)}(x)$ of $D_{2 k+1}$ are complex conjugate.

If $m_{n}=0$, then it follows from (4.20) that antisymmetric orbit functions of $D_{n}$ are real and can be represented as a sum of cosines of certain angles.

Explicit forms of antisymmetric orbit functions of $D_{n}$ are described by the following proposition [30]:

Proposition 5. Antisymmetric orbit functions of $D_{n}$ are representable in the form

$$
\begin{aligned}
\varphi_{\left(m_{1}, m_{2}, \ldots, m_{n}\right)}(x) & =\frac{1}{2} \operatorname{det}\left(e^{2 \pi \mathrm{i} m_{i} x_{j}}-e^{-2 \pi \mathrm{i} m_{i} x_{j}}\right)_{i, j=1}^{n}+\frac{1}{2} \operatorname{det}\left(e^{2 \pi \mathrm{i} m_{i} x_{j}}+e^{-2 \pi \mathrm{i} m_{i} x_{j}}\right)_{i, j=1}^{n} \\
& =\frac{1}{2}(2 \mathrm{i})^{n} \operatorname{det}\left(\sin 2 \pi m_{i} x_{j}\right)_{i, j=1}^{n}+2^{n-1} \operatorname{det}\left(\cos 2 \pi m_{i} x_{j}\right)_{i, j=1}^{n}
\end{aligned}
$$

if $m_{n} \neq 0$, and in the form

$$
\varphi_{\left(m_{1}, m_{2}, \ldots, m_{n}\right)}(x)=\frac{1}{2} \operatorname{det}\left(e^{2 \pi \mathrm{i} m_{i} x_{j}}+e^{-2 \pi \mathrm{i} m_{i} x_{j}}\right)_{i, j=1}^{n}=2^{n-1} \operatorname{det}\left(\cos 2 \pi m_{i} x_{j}\right)_{i, j=1}^{n}
$$

if $m_{n}=0$. 
Proof. Let $m_{n} \neq 0$. We take on the right hand side of (4.20) a sum of terms with fixed $w \in S_{n}$. It can be written as

$$
I_{w} \equiv(\operatorname{det} w) \sum_{\varepsilon_{i}= \pm 1}{ }^{\prime} \varepsilon_{1} \varepsilon_{2} \cdots \varepsilon_{n} e^{2 \pi \mathrm{i}\left((w(\varepsilon \lambda))_{1} x_{1}+\cdots+(w(\varepsilon \lambda))_{n} x_{n}\right)}
$$

where $\varepsilon_{1} \varepsilon_{2} \cdots \varepsilon_{n}=1$ (since there is an even number of $\varepsilon_{i}$ with $\varepsilon_{i}=-1$ ). A value of $I_{w}$ does not change if we add and subtract the same term to it:

$$
\begin{aligned}
I_{w}= & (\operatorname{det} w) \sum_{\varepsilon_{i}= \pm 1}^{\prime} \varepsilon_{1} \varepsilon_{2} \cdots \varepsilon_{n} e^{2 \pi \mathrm{i}\left((w(\varepsilon \lambda))_{1} x_{1}+\cdots+(w(\varepsilon \lambda))_{n} x_{n}\right)} \\
& +\frac{1}{2}(\operatorname{det} w) \sum_{\varepsilon_{i}= \pm 1}^{\prime \prime} \varepsilon_{1} \varepsilon_{2} \cdots \varepsilon_{n} e^{2 \pi \mathrm{i}\left((w(\varepsilon \lambda))_{1} x_{1}+\cdots+(w(\varepsilon \lambda))_{n} x_{n}\right)} \\
& -\frac{1}{2}(\operatorname{det} w) \sum_{\varepsilon_{i}= \pm 1}^{\prime \prime} \varepsilon_{1} \varepsilon_{2} \cdots \varepsilon_{n} e^{2 \pi \mathrm{i}\left((w(\varepsilon \lambda))_{1} x_{1}+\cdots+(w(\varepsilon \lambda))_{n} x_{n}\right)},
\end{aligned}
$$

the sum with two primes here means that the summation is over values of $\varepsilon_{i}$ with an odd number of negative $\varepsilon_{i}$. We split down the right hand side into two parts,

$$
I_{w}=\left[\frac{1}{2}(\operatorname{det} w) \sum_{\varepsilon_{i}= \pm 1}^{\prime} \cdots-\frac{1}{2}(\operatorname{det} w) \sum_{\varepsilon_{i}= \pm 1}^{\prime \prime} \cdots\right]+\left[\frac{1}{2}(\operatorname{det} w) \sum_{\varepsilon_{i}= \pm 1}^{\prime} \cdots+\frac{1}{2}(\operatorname{det} w) \sum_{\varepsilon_{i}= \pm 1}^{\prime \prime} \cdots\right]
$$

and repeat the reasoning of the proof of Proposition 3. As a result, we have

$$
\begin{aligned}
I_{w}= & \frac{1}{2}(\operatorname{det} w)\left(e^{2 \pi \mathrm{i}(w \lambda)_{1} x_{1}}-e^{-2 \pi \mathrm{i}(w \lambda)_{1} x_{1}}\right) \cdots\left(e^{2 \pi \mathrm{i}(w \lambda)_{n} x_{n}}-e^{-2 \pi \mathrm{i}(w \lambda)_{n} x_{n}}\right) \\
& +\frac{1}{2}(\operatorname{det} w)\left(e^{2 \pi \mathrm{i}(w \lambda)_{1} x_{1}}+e^{-2 \pi \mathrm{i}(w \lambda)_{1} x_{1}}\right) \cdots\left(e^{2 \pi \mathrm{i}(w \lambda)_{n} x_{n}}+e^{-2 \pi \mathrm{i}(w \lambda)_{n} x_{n}}\right) \\
= & \frac{1}{2}(\operatorname{det} w)(2 \mathrm{i})^{n} \sin 2 \pi(w \lambda)_{1} x_{1} \cdots \cdot \sin 2 \pi(w \lambda)_{n} x_{n} \\
& +\frac{1}{2}(\operatorname{det} w) 2^{n} \cos 2 \pi(w \lambda)_{1} x_{1} \cdots \cdot \cos 2 \pi(w \lambda)_{n} x_{n} .
\end{aligned}
$$

Then for $\varphi_{\lambda}(x)$ we have

$$
\begin{aligned}
\varphi_{\lambda}(x)= & \frac{1}{2}(2 \mathrm{i})^{n} \sum_{w \in S_{n}}(\operatorname{det} w) \sin 2 \pi(w \lambda)_{1} x_{1} \cdots \cdot \sin 2 \pi(w \lambda)_{n} x_{n} \\
& +\frac{1}{2} 2^{n} \sum_{w \in S_{n}}(\operatorname{det} w) \cos 2 \pi(w \lambda)_{1} x_{1} \cdots \cdot \cos 2 \pi(w \lambda)_{n} x_{n} \\
= & \frac{1}{2}(2 \mathrm{i})^{n} \operatorname{det}\left(\sin 2 \pi m_{i} x_{j}\right)_{i, j=1}^{n}+2^{n-1} \operatorname{det}\left(\cos 2 \pi m_{i} x_{j}\right)_{i, j=1}^{n},
\end{aligned}
$$

where $\lambda=\left(m_{1}, m_{2}, \ldots, m_{n}\right)$. Thus, the proposition is proved for the case $m_{n} \neq 0$.

Let $m_{n}=0$. Then, in the expression (4.20) for $\varphi_{\lambda}(x)$, in each term of the sum there exists the multiplier $e^{2 \pi \mathrm{i} \varepsilon_{n} m_{n} x_{i}}$ with some $i$. Since $m_{n}=0$ we have $e^{2 \pi \mathrm{i} \varepsilon_{n} m_{n} x_{i}}=1$ for $\varepsilon_{n}=1$ and for $\varepsilon_{n}=-1$. The case $\varepsilon_{n}=1$ gives an even number of negative $\varepsilon_{i}$ in the set $\left(\varepsilon_{1}, \varepsilon_{2}, \ldots, \varepsilon_{n-1}\right)$ and the case $\varepsilon_{n}=-1$ gives an odd number of negative $\varepsilon_{i}$ in this set. Therefore, we may throw out the sum over $\varepsilon_{n}$ and consider that summation in (4.20) runs over $\varepsilon_{i}= \pm 1, i=1,2, \ldots n-1$ (with even or odd number of negative $\varepsilon_{i}$ ):

$$
\varphi_{\lambda}(x)=\sum_{\varepsilon_{i}= \pm 1} \sum_{w \in S_{n}}(\operatorname{det} w) e^{2 \pi \mathrm{i}\left((w(\varepsilon \lambda))_{1} x_{1}+\cdots+(w(\varepsilon \lambda))_{n} x_{n}\right)}
$$

where in the sum the multipliers, containing $m_{n}$, are removed and the first sum does not contain summation over $\varepsilon_{n}$. As in the case of the proof of Proposition 3, we take in (4.23) terms with fixed $w$ and write down it as

$$
(\operatorname{det} w) \sum_{\varepsilon_{i}= \pm 1} e^{2 \pi \mathrm{i}\left((w(\varepsilon \lambda))_{1} x_{1}+\cdots+(w(\varepsilon \lambda))_{n} x_{n}\right)}
$$




$$
\begin{aligned}
& =\frac{1}{2}(\operatorname{det} w)\left(e^{2 \pi \mathrm{i}(w \lambda)_{1} x_{1}}+e^{-2 \pi \mathrm{i}(w \lambda)_{1} x_{1}}\right) \cdots\left(e^{2 \pi \mathrm{i}(w \lambda)_{n} x_{n}}+e^{-2 \pi \mathrm{i}(w \lambda)_{n} x_{n}}\right) \\
& =2^{n-1}(\operatorname{det} w) \cos 2 \pi(w \lambda)_{1} x_{1} \cdots \cdot \cos 2 \pi(w \lambda)_{n} x_{n},
\end{aligned}
$$

where, as before, the multipliers, containing $m_{n}$, are omitted. Since $e^{2 \pi \mathrm{i} m_{n} x_{i}}+e^{-2 \pi \mathrm{i} m_{n} x_{i}}=2$, this leads to the formula (4.22) for $\varphi_{\lambda}(x)$ when $m_{n}=0$. The proposition is proved.

For the antisymmetric orbit function $\varphi_{\rho}, \rho=\frac{1}{2} \sum_{\alpha>0} \alpha$, one has

$$
\varphi_{\rho}(x)=2^{n-1} \operatorname{det}\left(\cos 2 \pi \rho_{i} x_{j}\right)_{i, j=1}^{n}
$$

where $\rho=\left(\rho_{1}, \rho_{2}, \ldots, \rho_{n}\right)=(n-1, n-2, \ldots, 1,0)$.

\subsection{Symmetric orbit functions of $B_{n}, C_{n}$ and $D_{n}$}

In Subsections 4.5-4.7 we have derived expressions for antisymmetric orbit functions $\varphi_{\lambda}(x)$ of $B_{n}, C_{n}$ and $D_{n}$ in orthogonal coordinates as determinants of sine and cosine functions. Similar expressions can be derived for symmetric orbit functions of $B_{n}, C_{n}$ and $D_{n}$. Since in the defining expressions for symmetric orbit functions $\phi_{\lambda}(x)$ (see formula (4.1)) there are no multipliers $(\operatorname{det} w)$, then $\phi_{\lambda}(x)$ is expressed in terms of products of sine and cosine functions (instead of determinants).

As before, we express elements $\lambda=\left(m_{1}, m_{2}, \ldots, m_{n}\right)$ and $x=\left(x_{1}, x_{2}, \ldots, x_{n}\right)$ in the corresponding orthogonal coordinate systems. In Propositions 6 and 7 below we suppose that $\lambda$ is an element of the dominant Weyl chamber and is not obligatory integral.

Proposition 6. Symmetric orbit functions of $B_{n}$ and $C_{n}$ can be represented in the form

$$
\phi_{\left(m_{1}, m_{2}, \ldots, m_{n}\right)}(x)=2^{n} \sum_{w \in S_{n}} \cos 2 \pi m_{w(1)} x_{1} \cdots \cos 2 \pi m_{w(n)} x_{n},
$$

where $w(1), w(2), \ldots, w(n)$ is the set of numbers $1,2, \ldots, n$ obtained by acting by the permutation $w \in S_{n}$.

This proposition is proved in the same way as Proposition 3 and we omit it.

Proposition 7. Symmetric orbit functions of $D_{n}$ can be represented in the form

$$
\begin{aligned}
\phi_{\left(m_{1}, m_{2}, \ldots, m_{n}\right)}(x)= & 2^{n-1} \sum_{w \in S_{n}} \cos 2 \pi m_{w(1)} x_{1} \cdots \cdot \cos 2 \pi m_{w(n)} x_{n} \\
& +\frac{1}{2}(2 \mathrm{i})^{n} \sum_{w \in S_{n}} \sin 2 \pi m_{w(1)} x_{1} \cdots \cdot \sin 2 \pi m_{w(n)} x_{n}
\end{aligned}
$$

if $m_{n} \neq 0$ and in the form

$$
\varphi_{\left(m_{1}, m_{2}, \ldots, m_{n}\right)}(x)=2^{n-1} \sum_{w \in S_{n}} \cos 2 \pi m_{w(1)} x_{1} \cdots \cdot \cos 2 \pi m_{w(n)} x_{n}
$$

if $m_{n}=0$, where $w(1), w(2), \ldots, w(n)$ is the set of numbers $1,2, \ldots, n$ obtained by acting by the permutation $s \in S_{n}$.

A proof is similar to the proof of Proposition 5. 


\section{Properties of antisymmetric orbit functions}

\subsection{Anti-invariance with respect to the Weyl group}

Since the scalar product $\langle\cdot, \cdot\rangle$ in $E_{n}$ is invariant with respect to the Weyl group $W$, that is,

$$
\langle w x, w y\rangle=\langle x, y\rangle, \quad w \in W, \quad x, y \in E_{n},
$$

antisymmetric orbit functions $\varphi_{\lambda}$ for strictly positive elements $\lambda$ (not obligatory belonging to $P_{+}^{+}$) are anti-invariant with respect to $W$, that is,

$$
\varphi_{\lambda}\left(w^{\prime} x\right)=\left(\operatorname{det} w^{\prime}\right) \varphi_{\lambda}(x), \quad w^{\prime} \in W .
$$

Indeed,

$$
\begin{aligned}
\varphi_{\lambda}\left(w^{\prime} x\right) & =\sum_{w \in W}(\operatorname{det} w) e^{2 \pi \mathrm{i}\left\langle w \lambda, w^{\prime} x\right\rangle}=\sum_{w \in W}(\operatorname{det} w) e^{2 \pi \mathrm{i}\left\langle w^{\prime-1} w \lambda, x\right\rangle} \\
& =\left(\operatorname{det} w^{\prime}\right) \sum_{w \in W}(\operatorname{det} w) e^{2 \pi \mathrm{i}\langle w \lambda, x\rangle}=\left(\operatorname{det} w^{\prime}\right) \varphi_{\lambda}(x)
\end{aligned}
$$

since $w^{-1} w$ runs over the whole group $W$ when $w$ runs over $W$.

\subsection{Anti-invariance with respect to the affine Weyl group}

The affine Weyl group $W^{\text {aff }}$ is generated by reflections $r_{0}, r_{\alpha_{1}}, \ldots, r_{\alpha_{n}}$ (see Subsection 2.5). We say that an antisymmetric orbit function $\varphi_{\lambda}, \lambda \in P_{+}^{+}$, is anti-invariant with respect to the affine Weyl group $W^{\text {aff }}$ if $\varphi_{\lambda}\left(r_{0} x\right)=-\varphi_{\lambda}(x)$. Let us show that $\varphi_{\lambda}(x)$ satisfies this relation. Since $r_{0} x=r_{\xi} x+\xi^{\vee}$, where $\xi$ is the highest root (see Subsection 2.4), for $\mu \in P$ we have

$$
\left\langle\mu, r_{0} x\right\rangle=\left\langle\mu, \xi^{\vee}+r_{\xi} x\right\rangle=\frac{2\langle\mu, \xi\rangle}{\langle\xi, \xi\rangle}+\left\langle\mu, r_{\xi} x\right\rangle=\text { integer }+\left\langle r_{\xi} \mu, x\right\rangle
$$

since $r_{\xi}^{2}=1$. Hence,

$$
\begin{aligned}
\varphi_{\lambda}\left(r_{0} x\right) & =\sum_{w \in W}(\operatorname{det} w) e^{2 \pi \mathrm{i}\left\langle w \lambda, r_{0} x\right\rangle}=\sum_{w \in W}(\operatorname{det} w) e^{2 \pi \mathrm{i}\left\langle r_{\xi} w \lambda, x\right\rangle} \\
& =\left(\operatorname{det} r_{\xi}\right) \sum_{w \in W}(\operatorname{det} w) e^{2 \pi \mathrm{i}\langle w \lambda, x\rangle}=-\varphi_{\lambda}(x)
\end{aligned}
$$

since $r_{\xi}$ is a reflection belonging to $W$.

If $\lambda \neq P$, then $\varphi_{\lambda}$ is not anti-invariant with respect to $r_{0}$. It is anti-invariant only under action by elements of the Weyl group $W$.

Due to the anti-invariance of antisymmetric orbit functions $\varphi_{\lambda}, \lambda \in P_{+}^{+}$, with respect to the group $W^{\text {aff }}$, it is enough to consider them only on the fundamental domain $F \equiv F\left(W^{\text {aff }}\right)$ of $W^{\text {aff }}$. Values of $\varphi_{\lambda}$ on other points of $E_{n}$ are determined by using the action of $W^{\text {aff }}$ on $F$ or taking a limit. In particular, functions $\varphi_{\lambda}, \lambda \in P_{+}^{+}$, are anti-invariant under a reflection with respect to any $(n-1)$-dimensional wall of the fundamental domain $F$.

\subsection{Continuity and vanishing}

An antisymmetric orbit function $\varphi_{\lambda}$ is a finite sum of exponential functions. Therefore it is continuous and has continuous derivatives of all orders in $E_{n}$.

Due to anti-invariance of the orbit functions $\varphi_{\lambda}, \lambda \in D_{+}^{+}$, with respect to the Weyl group $W$, $\varphi_{\lambda}$ vanishes on all walls of the Weyl chambers. The anti-invariance $\varphi_{\lambda}\left(r_{0} x\right)=-\varphi_{\lambda}(x)$ for $\lambda \in P_{+}^{+}$shows that $\varphi_{\lambda}, \lambda \in P_{+}^{+}$, vanishes on the boundary of the fundamental domain $F$ of the affine Weyl group $W^{\text {aff }}$. 


\subsection{Realness and complex conjugation}

The results, formulated below and concerning antisymmetric orbit functions of the CoxeterDynkin diagrams $A_{n}, B_{n}, C_{n}, D_{n}$ and $G_{2}$, were proved in the previous section.

Antisymmetric orbit functions of the following Coxeter-Dynkin diagrams are real:

$$
B_{2 k}, \quad C_{2 k}, \quad G_{2} \text {. }
$$

Antisymmetric orbit functions of the Coxeter-Dynkin diagrams $B_{2 k+1}$ and $C_{2 k+1}$ are purely imaginary. The antisymmetric orbit functions $\varphi_{\lambda}$ of the Coxeter-Dynkin diagrams $A_{4 k-1}$ and $A_{4 k}$ satisfy the condition

$$
\varphi_{\left(\lambda_{1}, \lambda_{2}, \ldots, \lambda_{n}\right)}(x)=\overline{\varphi_{\left(\lambda_{n}, \lambda_{n-1}, \ldots, \lambda_{1}\right)}(x)}
$$

and antisymmetric orbit functions $\varphi_{\lambda}$ of the Coxeter-Dynkin diagrams $A_{4 k+1}$ and $A_{4 k+2}$ satisfy the condition

$$
\varphi_{\left(\lambda_{1}, \lambda_{2}, \ldots, \lambda_{n}\right)}(x)=-\overline{\varphi_{\left(\lambda_{n}, \lambda_{n-1}, \ldots, \lambda_{1}\right)}(x)} .
$$

Antisymmetric orbit functions $\varphi_{\left(\lambda_{1}, \lambda_{2}, \ldots, \lambda_{n}\right)}$ of $D_{n}$ are real if $\lambda_{n-1}=\lambda_{n}$. If $\lambda_{n-1} \neq \lambda_{n}$, then orbit functions $\varphi_{\left(\lambda_{1}, \lambda_{2}, \ldots, \lambda_{n}\right)}$ are real for $n=2 k$ and satisfy the condition

$$
\varphi_{\left(\lambda_{1}, \ldots, \lambda_{n-2}, \lambda_{n-1}, \lambda_{n}\right)}=\overline{\varphi\left(\lambda_{1}, \ldots, \lambda_{n-2}, \lambda_{n}, \lambda_{n-1}\right)}
$$

if $n=2 k+1$.

\subsection{Scaling symmetry}

Let $O(\lambda)$ be an orbit of $\lambda, \lambda \in D_{+}^{+}$. Since $w(c \lambda)=c w(\lambda)$ for any $c \in \mathbb{R}$ and for any $w \in W$, then the orbit $O(c \lambda)$ is an orbit consisting of the points $c w \lambda, w \in W$. Moreover, points $w \lambda$ and $c w \lambda$ of the signed orbits $O^{ \pm}(\lambda)$ and $O^{ \pm}(c \lambda)$, respectively, have the same sign. Let $\varphi_{\lambda}=$ $\sum_{w \in W}(\operatorname{det} w) e^{2 \pi \mathrm{i} w \lambda}$ be the antisymmetric orbit function for $\lambda \in D_{+}^{+}$. Then

$$
\varphi_{c \lambda}(x)=\sum_{w \in W}(\operatorname{det} w) e^{2 \pi \mathrm{i}\langle c w \lambda, x\rangle}=\sum_{w \in W}(\operatorname{det} w) e^{2 \pi \mathrm{i}\langle w \lambda, c x\rangle}=\varphi_{\lambda}(c x) .
$$

The equality $\varphi_{c \lambda}(x)=\varphi_{\lambda}(c x)$ expresses the scaling symmetry of orbit functions.

If we consider only antisymmetric orbit functions $\varphi_{\lambda}$, corresponding to $\lambda \in P_{+}^{+}$, then the scaling symmetry $\varphi_{c \lambda}(x)=\varphi_{\lambda}(c x)$ holds for those values $c \in \mathbb{R} \backslash\{0\}$ for which $c \lambda \in P_{+}^{+}$.

\subsection{Duality}

Due to the invariance of the scalar product $\langle\cdot, \cdot\rangle$ with respect to the Weyl group $W,\langle w \mu, w y\rangle=$ $\langle\mu, y\rangle$, for $x \in E_{n}$ not lying on a wall of some Weyl chamber we have

$$
\varphi_{\lambda}(x)=\sum_{w \in W}(\operatorname{det} w) e^{2 \pi \mathrm{i}\left\langle\lambda, w^{-1} x\right\rangle}=\sum_{w \in W}(\operatorname{det} w) e^{2 \pi \mathrm{i}\langle\lambda, w x\rangle}=\varphi_{x}(\lambda) .
$$

The relation $\varphi_{\lambda}(x)=\varphi_{x}(\lambda)$ expresses the duality of antisymmetric orbit functions. 


\subsection{Orthogonality}

Antisymmetric orbit functions $\varphi_{\lambda}, \lambda \in P_{+}^{+}$, are orthogonal on $F$ with respect to the Euclidean measure [31]:

$$
|F|^{-1} \int_{F} \varphi_{\lambda}(x) \overline{\varphi_{\lambda^{\prime}}(x)} d x=|W| \delta_{\lambda \lambda^{\prime}}
$$

where the overbar means complex conjugation. This relation directly follows from orthogonality of the exponential functions $e^{2 \pi \mathrm{i}\langle\mu, x\rangle}$ (entering into the definition of orbit functions) for different weights $\mu$ and from the fact that a given weight $\nu \in P$ belongs to precisely one orbit function. In (5.1), $|F|$ means an area of the fundamental domain $F$.

Sometimes, it is difficult to find the area $|F|$. In this case it is useful the following form of the formula (5.1):

$$
\int_{\mathrm{T}} \varphi_{\lambda}(x) \overline{\varphi_{\lambda^{\prime}}(x)} d x=|W| \delta_{\lambda \lambda^{\prime}}
$$

where $\mathrm{T}$ is the torus in $E_{n}$ described in Subsection 9.1 below. If to assume that an area of $\mathrm{T}$ is equal to $1,|\mathrm{~T}|=1$, then $|F|=|W|^{-1}$ and formula (5.1) takes the form

$$
\int_{F} \varphi_{\lambda}(x) \overline{\varphi_{\lambda^{\prime}}(x)} d x=\delta_{\lambda \lambda^{\prime}}
$$

The formula (5.2) gives the orthogonality relation for the antisymmetric multivariate sine function (4.19). We have

$$
2^{2 n} \int_{F} \operatorname{det}\left(\sin 2 \pi m_{i} x_{j}\right)_{i, j=1}^{n} \operatorname{det}\left(\sin 2 \pi m_{i}^{\prime} x_{j}\right)_{i, j=1}^{n} d x=\delta_{\mathbf{m}, \mathbf{m}^{\prime}},
$$

where $\mathbf{m}=\left(m_{1}, m_{2}, \ldots, m_{n}\right)$ and $\mathbf{m}^{\prime}=\left(m_{1}^{\prime}, m_{2}^{\prime}, \ldots, m_{n}^{\prime}\right)$ are strictly dominant and integral (that is, $m_{i}, m_{j}^{\prime} \in \mathbb{Z}^{>0}, m_{1}>m_{2}>\cdots>m_{n}>0$ ), and the domain $F$ consists of points $x=\left(x_{1}, x_{2}, \ldots, x_{n}\right) \in E_{n}$ such that

$$
\frac{1}{2} \geq x_{1} \geq x_{2} \geq \cdots \geq x_{n} \geq 0
$$

(see Subsection 5.10 below).

A similar orthogonality relation can be written down for the symmetric multivariate cosine function (4.24). Introducing the notation

$$
\operatorname{det}^{+}\left(\cos 2 \pi m_{i} x_{j}\right)_{i, j=1}^{n}:=\sum_{w \in S_{n}} \cos 2 \pi m_{w(1)} x_{1} \cdots \cdot \cos 2 \pi m_{w(n)} x_{n}
$$

we have

$$
2^{2 n} \int_{F} \operatorname{det}^{+}\left(\cos 2 \pi m_{i} x_{j}\right)_{i, j=1}^{n} \operatorname{det}^{+}\left(\cos 2 \pi m_{i}^{\prime} x_{j}\right)_{i, j=1}^{n} d x=\left|W_{\mathbf{m}}\right| \delta_{\mathbf{m}, \mathbf{m}^{\prime}}
$$

where $\mathbf{m}$ and $\mathbf{m}^{\prime}$ are such that $m_{1} \geq m_{2} \geq \cdots \geq m_{n}, m_{1}^{\prime} \geq m_{2}^{\prime} \geq \cdots \geq m_{n}^{\prime}$, and $\left|W_{\mathbf{m}}\right|$ is an order of the subgroup $\left|W_{\mathbf{m}}\right| \subset S_{n}$ consisting of elements leaving $\mathbf{m}$ invariant. 


\subsection{Orthogonality to symmetric orbit functions}

Let $\alpha_{i}$ be a simple root. We create the domain $F^{\text {ext }}=F \cup r_{\alpha_{i}} F$, where $r_{\alpha_{i}}$ is the reflection corresponding to the root $\alpha_{i}$ and $F$ is the fundamental domain. Since for $\mu \in P_{+}^{+}$we have

$$
\phi_{\mu}\left(r_{\alpha_{i}} x\right)=\phi_{\mu}(x), \quad \varphi_{\mu}\left(r_{\alpha_{i}} x\right)=-\varphi_{\mu}(x),
$$

where $\phi_{\mu}$ is a symmetric orbit function, then

$$
\int_{F_{\mathrm{ext}}} \phi_{\mu}(x) \overline{\varphi_{\mu}(x)} d x=0 .
$$

Indeed, due to symmetry and antisymmetry of symmetric and antisymmetric orbit functions we have

$$
\begin{aligned}
\int_{F \mathrm{ext}} \phi_{\mu}(x) \overline{\varphi_{\mu}(x)} d x & =\int_{F} \phi_{\mu}(x) \overline{\varphi_{\mu}(x)} d x+\int_{r_{\alpha_{i}} F} \phi_{\mu}(x) \overline{\varphi_{\mu}(x)} d x \\
& \left.=\int_{F} \phi_{\mu}(x) \overline{\varphi_{\mu}(x)} d x+\int_{F} \phi_{\mu}(x) \overline{\left(-\varphi_{\mu}(x)\right.}\right) d x=0 .
\end{aligned}
$$

For the case of $A_{1}$ the orthogonality (5.6) means the orthogonality of the functions sine and cosine on the interval $(0,2 \pi)$.

The formula (5.6) determines orthogonality of multivariate sine and cosine functions (4.19) and (5.4):

$$
\int_{F^{\mathrm{ext}}} \operatorname{det}\left(\sin 2 \pi m_{i} x_{j}\right)_{i, j=1}^{n} \operatorname{det}^{+}\left(\cos 2 \pi m_{i} x_{j}\right)_{i, j=1}^{n} d x=0,
$$

where the notations are such as in (5.3) and $F^{\text {ext }}$ consists of points $x \in E_{n}$ such that

$$
\frac{1}{2} \geq x_{1} \geq x_{2} \geq \cdots \geq x_{n} \geq 0 \quad \text { or } \quad \frac{1}{2} \geq x_{2} \geq x_{1} \geq x_{3} \geq x_{4} \geq \cdots \geq x_{n} \geq 0 .
$$

\section{$5.9 \quad$ Antisymmetric orbit functions $\varphi_{\rho}$}

Let $\rho$ be the half-sum of all positive roots of a root system:

$$
\rho=\frac{1}{2} \sum_{\alpha>0} \alpha
$$

It is well-known that for all simple Lie algebras in $\omega$-coordinate we have

$$
\rho=\omega_{1}+\omega_{2}+\cdots+\omega_{n} \equiv\left(\begin{array}{llll}
1 & 1 & \cdots & 1
\end{array}\right) .
$$

The antisymmetric orbit function $\varphi_{\rho}$ is important in the theory of characters of group representations. We have

$$
\varphi_{\rho}(x)=\sum_{w \in W}(\operatorname{det} w) e^{2 \pi \mathrm{i}\langle w \rho, x\rangle} .
$$

Proposition 8. The antisymmetric orbit function $\varphi_{\rho}$ can be represented as

$$
\begin{aligned}
\varphi_{\rho}(x) & =\prod_{\alpha>0}\left(e^{\pi \mathrm{i}\langle\alpha, x\rangle}-e^{-\pi \mathrm{i}\langle\alpha, x\rangle}\right)=e^{2 \pi \mathrm{i}\langle\rho, x\rangle} \prod_{\alpha>0}\left(1-e^{-2 \pi \mathrm{i}\langle\alpha, x\rangle}\right) \\
& =(2 \mathrm{i})^{r} \prod_{\alpha>0} \sin \pi\langle\alpha, x\rangle,
\end{aligned}
$$

where $r$ is a number of positive roots in the corresponding root system. 
Proof. The expression $g(x)=\prod_{\alpha>0}\left(e^{\pi \mathrm{i}\langle\alpha, x\rangle}-e^{-\pi \mathrm{i}\langle\alpha, x\rangle}\right)$ is antisymmetric with respect to the Weyl group $W$. Indeed, if $r_{i}$ is a reflection, corresponding to the simple root $\alpha_{i}$, then due to Proposition 1 we have

$$
\begin{aligned}
g\left(r_{i} x\right) & =\prod_{\alpha>0}\left(e^{\pi \mathrm{i}\left\langle\alpha, r_{i} x\right\rangle}-e^{-\pi \mathrm{i}\left\langle\alpha, r_{i} x\right\rangle}\right)=\prod_{\alpha>0}\left(e^{\pi \mathrm{i}\left\langle r_{i} \alpha, x\right\rangle}-e^{-\pi \mathrm{i}\left\langle r_{i} \alpha, x\right\rangle}\right) \\
& =\left(e^{-\pi \mathrm{i}\left\langle\alpha_{i}, x\right\rangle}-e^{\pi \mathrm{i}\left\langle\alpha_{i}, x\right\rangle}\right) \prod_{\alpha>0, \alpha \neq \alpha_{i}}\left(e^{\pi \mathrm{i}\langle\alpha, x\rangle}-e^{-\pi \mathrm{i}\langle\alpha, x\rangle}\right)=-g(x) .
\end{aligned}
$$

Since $\alpha_{i}$ is an arbitrary simple root, $g(x)$ is antisymmetric with respect to $W$. Then $g(x)$ is a sum of antisymmetric orbit functions. Representing $\prod_{\alpha>0}\left(e^{\pi \mathrm{i}\langle\alpha, x\rangle}-e^{-\pi \mathrm{i}\langle\alpha, x\rangle}\right)$ in the form (4.3), we see that in this form there exists only one term $e^{\pi \mathrm{i}\langle\lambda, x\rangle}$ with a strictly dominant weight $\lambda$ and this weight coincides with $\rho$. Therefore, $g(x)=\varphi_{\rho}(x)$. Proposition is proved.

An invariant measure of the compact Lie group $G$, associated with the Weyl group $W$, is expressed in terms of $\left|\varphi_{\rho}\right|^{2}$. Taking into account an explicit form of positive roots in the orthogonal coordinate systems, it is easy to derive from (5.9) that in these coordinates we have

$$
\begin{aligned}
& \varphi_{\rho}(x)=(2 \mathrm{i})^{n(n+1) / 2} \prod_{1 \leq i<j \leq n+1} \sin \pi\left(x_{i}-x_{j}\right) \quad \text { for } A_{n}, \\
& \varphi_{\rho}(x)=(2 \mathrm{i})^{n^{2}} \prod_{1 \leq i<j \leq n} \sin \pi\left(x_{i}-x_{j}\right) \sin \pi\left(x_{i}+x_{j}\right) \prod_{1 \leq i \leq n} \sin \pi x_{i} \quad \text { for } B_{n}, \\
& \varphi_{\rho}(x)=(2 \mathrm{i})^{n^{2}} \prod_{1 \leq i<j \leq n} \sin \pi\left(x_{i}-x_{j}\right) \sin \pi\left(x_{i}+x_{j}\right) \prod_{1 \leq i \leq n} \sin 2 \pi x_{i} \quad \text { for } C_{n}, \\
& \varphi_{\rho}(x)=(2 \mathrm{i})^{n(n-1)} \prod_{1 \leq i<j \leq n} \sin \pi\left(x_{i}-x_{j}\right) \sin \pi\left(x_{i}+x_{j}\right) \quad \text { for } D_{n} .
\end{aligned}
$$

These formulas give other expressions for $\left|\varphi_{\rho}(x)\right|$ with respect to formulas derived in Subsections 4.4-4.7.

Proposition 9. The antisymmetric orbit function $\varphi_{\rho}$ vanishes on the boundary of the fundamental domain $F$. It does not vanish on intrinsic points of $F$.

Proof. Since $\rho \in P_{+}^{+}$, then $\varphi_{\rho}$ vanishes on the boundary of $F$ due to the results of Subsection 5.3. From the other side, it is easy to see from (5.9) that the set of points, on which $\varphi_{\rho}(x)$ vanishes, coincides with the set of all points $x \in E_{n}$ for which $\langle\alpha, x\rangle \in \mathbb{Z}$ for some root $\alpha$. No of these points is an intrinsic point of $F$. The proposition is proved.

From Proposition 9 and from the above formulas for $\varphi_{\rho}(x)$ we easily derive explicit forms of the fundamental domains for the cases $A_{n}, B_{n}, C_{n}, D_{n}$ in the orthogonal coordinates.

(a) The fundamental domain $F\left(A_{n}\right)$ is contained in the domain of real points $x=\left(x_{1}, x_{2}, \ldots\right.$, $\left.x_{n+1}\right)$ such that

$$
x_{1}>x_{2}>\cdots>x_{n+1}, \quad x_{1}+x_{2}+\cdots+x_{n+1}=0 .
$$

Moreover, a point $x$ of this domain belongs to $F\left(A_{n}\right)$ if and only if $x_{1}+\left|x_{n+1}\right|<1$. The last condition means in fact the relation $\langle x, \xi\rangle<1$ for the highest root $\xi$ of the root system $A_{n}$.

(b) The fundamental domain $F\left(B_{n}\right)$ is contained in the domain of points $x=\left(x_{1}, x_{2}, \ldots\right.$, $x_{n}$ ) such that

$$
1>x_{1}>x_{2}>\cdots>x_{n}>0
$$


Moreover, a point $x$ of this domain belongs to $F\left(B_{n}\right)$ if and only if $x_{1}+x_{2}<1$.

(c) The fundamental domain $F\left(C_{n}\right)$ consists of all points $x=\left(x_{1}, x_{2}, \ldots, x_{n}\right)$ such that

$$
\frac{1}{2}>x_{1}>x_{2}>\cdots>x_{n}>0 .
$$

(d) The fundamental domain $F\left(D_{n}\right)$ is contained in the domain of points $x=\left(x_{1}, x_{2}, \ldots\right.$, $\left.x_{n}\right)$ such that

$$
1>x_{1}>x_{2}>\cdots>x_{n-1}>\left|x_{n}\right| \text {. }
$$

Moreover, a point $x$ of this domain belongs to $F\left(D_{n}\right)$ if and only if $x_{1}+x_{2}<1$.

\subsection{Symmetric orbit functions $\phi_{\rho}$}

Let us consider a symmetric counterpart of the antisymmetric orbit function $\varphi_{\rho}(x)$ :

$$
\phi_{\rho}(x):=\sum_{w \in W} e^{2 \pi \mathrm{i}\langle w \rho, x\rangle}
$$

where, as before, $\rho=\frac{1}{2} \sum_{\alpha>0} \alpha$.

Proposition 10. The symmetric orbit function $\phi_{\rho}$ can be represented as

$$
\begin{aligned}
\varphi_{\rho}(x) & =\prod_{\alpha>0}\left(e^{\pi \mathrm{i}\langle\alpha, x\rangle}+e^{-\pi \mathrm{i}\langle\alpha, x\rangle}\right)=e^{2 \pi \mathrm{i}\langle\rho, x\rangle} \prod_{\alpha>0}\left(1+e^{-2 \pi \mathrm{i}\langle\alpha, x\rangle}\right) \\
& =2^{r} \prod_{\alpha>0} \cos \pi\langle\alpha, x\rangle,
\end{aligned}
$$

where $r$ is a number of positive roots in the corresponding root system.

This proposition is proved in the same way as Proposition 6 .

It is easy to derive from (5.11) that in the orthogonal coordinate systems we have

$$
\begin{array}{lll}
\phi_{\rho}(x) & =2^{n(n+1) / 2} \prod_{1 \leq i<j \leq n+1} \cos \pi\left(x_{i}-x_{j}\right) \quad \text { for } A_{n}, & \\
\phi_{\rho}(x)=2^{n^{2}} \prod_{1 \leq i<j \leq n} \cos \pi\left(x_{i}-x_{j}\right) \cos \pi\left(x_{i}+x_{j}\right) \prod_{1 \leq i \leq n} \cos \pi x_{i} & \text { for } B_{n}, \\
\phi_{\rho}(x)=2^{n^{2}} \prod_{1 \leq i<j \leq n} \cos \pi\left(x_{i}-x_{j}\right) \cos \pi\left(x_{i}+x_{j}\right) \prod_{1 \leq i \leq n} \cos 2 \pi x_{i} & \text { for } C_{n}, \\
\phi_{\rho}(x)=2^{n(n-1)} \prod_{1 \leq i<j \leq n} \cos \pi\left(x_{i}-x_{j}\right) \cos \pi\left(x_{i}+x_{j}\right) & \text { for } D_{n} .
\end{array}
$$

\section{Properties of antisymmetric orbit functions of $A_{n}$}

By using results on decomposition of certain reducible representations of the group $G L(n, \mathbb{C})$ into irreducible constituents, properties of antisymmetric orbit functions of $A_{n}$ can be derived. We use in this section the results of [30]. 


\subsection{Decomposition of symmetric powers of representations}

We need some formulas for decomposition of symmetric powers of finite dimensional irreducible representations of the group $G L\left(n_{1}, \mathbb{C}\right) \times G L\left(n_{2}, \mathbb{C}\right)$. Let us first define symmetric powers of representations.

Let $T$ be a finite dimensional representation of a group $G$ on a linear space $X$. It induces a representation on the space $\mathcal{P}_{m}(X)$, which is a subspace of the space $\mathcal{P}(X)$ of polynomials on $X$, consisting of all homogeneous polynomials of power $m$. In order to determine this representation we note that the formula

$$
(Q(g) p)(x)=p\left(T\left(g^{-1}\right) x\right), \quad x \in X, \quad g \in G,
$$

gives a representation of $G$ on $\mathcal{P}(X)$ which is denoted by $Q$. The subspace $\mathcal{P}_{m}(X)$ is invariant with respect to the representation $Q$. The restriction of $Q$ onto $\mathcal{P}_{m}(X)$ is called an $m$-th symmetric power of the representation $T$ and is denoted as $\sigma_{m}(T)$.

Let $G=G L\left(n_{1}, \mathbb{C}\right) \times G L\left(n_{2}, \mathbb{C}\right), n_{1} \leq n_{2}$. Then a finite dimensional irreducible representation of $G$ is given (in the orthogonal coordinate system) by

$$
(\lambda \mid \mu)=\left(m_{1}, m_{2}, \ldots, m_{n_{1}} \mid r_{1}, r_{2}, \ldots, r_{n_{2}}\right),
$$

where

$$
m_{1} \geq m_{2} \geq \cdots \geq m_{n_{1}}, \quad r_{1} \geq r_{2} \geq \cdots \geq r_{n_{2}} .
$$

We assume that $m_{n_{1}} \geq 0$ and $r_{n_{2}} \geq 0$.

One can consider three representations of $G=G L\left(n_{1}, \mathbb{C}\right) \times G L\left(n_{2}, \mathbb{C}\right)$ on the space $\mathfrak{M}_{n_{1} n_{2}}(\mathbb{C})$ of $n_{1} \times n_{2}$ complex matrices. They are given by the formulas

$$
\begin{aligned}
& T\left(g_{1}, g_{2}\right) X=g_{1} X g_{2}^{t}, \\
& T^{\prime}\left(g_{1}, g_{2}\right) X=g_{1} X g_{2}^{*}, \\
& T^{\prime \prime}\left(g_{1}, g_{2}\right) X=g_{1} X g_{2}^{-1},
\end{aligned}
$$

where the index $t$ means a transposition and $*$ means a transposition together with complex conjugation. Then for symmetric powers of these representations we have the following decompositions into irreducible representations of $G$ (a proof see in [30]):

$$
\begin{aligned}
\sigma_{m}(T) & =\sum_{\sum_{i} s_{i}=m}\left(s_{1}, s_{2}, \ldots, s_{n_{1}} \mid s_{1}, s_{2}, \ldots, s_{n_{1}}, 0, \ldots, 0\right), \\
\sigma_{m}\left(T^{\prime}\right) & =\sum_{\sum_{i} s_{i}=m}\left(s_{1}, s_{2}, \ldots, s_{n_{1}} \mid \overline{s_{1}, s_{2}, \ldots, s_{n_{1}}, 0, \cdots, 0}\right), \\
\sigma_{m}\left(T^{\prime \prime}\right) & =\sum_{\sum_{i} s_{i}=m}\left(s_{1}, s_{2}, \ldots, s_{n_{1}} \mid 0, \ldots, 0,-s_{n_{1}},-s_{n_{2}}, \ldots,-s_{1}\right),
\end{aligned}
$$

where summations are over all $s_{1}, s_{2}, \ldots, s_{n_{1}}$ such that

$$
s_{1} \geq s_{2} \geq \cdots \geq s_{n_{1}} \geq 0, \quad s_{1}+s_{2}+\cdots+s_{n_{1}}=m
$$

and the overbar means that a representation is anti-analytic.

If $n_{1}=n_{2}=n$, then the formulas (6.1)-(6.3) determine the following tensor product representations of $G=G L(n, \mathbb{C})$ on the space $\mathfrak{M}_{n}(\mathbb{C})$ of complex $n \times n$ matrices:

$$
\left(T_{1} \otimes T_{1}\right)(g) X=g X g^{t},
$$




$$
\begin{aligned}
& \left(T_{1} \otimes \bar{T}_{1}\right)(g) X=g X g^{*}, \\
& \left(T_{1} \otimes \hat{T}_{1}\right)(g) X=g X g^{-1},
\end{aligned}
$$

where $T_{1}$ is the first fundamental representation (with highest weight $(1,0, \ldots, 0)$ ) of $G=$ $G L(n, \mathbb{C}), \bar{T}_{1}$ and $\hat{T}_{1}$ are the complex conjugate and contragredient representations to the representation $T_{1}$, respectively. Then according to (6.4)-(6.6) we have

$$
\begin{aligned}
\sigma_{m}\left(T_{1} \otimes T_{1}\right) & =\sum_{\mathbf{m}} T_{\mathbf{m}} \otimes T_{\mathbf{m}}, \\
\sigma_{m}\left(T_{1} \otimes \bar{T}_{1}\right) & =\sum_{\mathbf{m}} T_{\mathbf{m}} \otimes \bar{T}_{\mathbf{m}}, \\
\sigma_{m}\left(T_{1} \otimes T_{1}\right) & =\sum_{\mathbf{m}} T_{\mathbf{m}} \otimes \hat{T}_{\mathbf{m}},
\end{aligned}
$$

where $T_{\mathbf{m}}$ is the irreducible representation of the group $G L(n, \mathbb{C})$ with highest weight $\mathbf{m} \equiv$ $\left(m_{1}, m_{2}, \ldots, m_{n}\right), m_{1} \geq m_{2} \geq \cdots \geq m_{n} \geq 0$, and the summation is over those $\mathbf{m}$ for which $m_{1}+m_{2}+\cdots+m_{n}=m$.

Replacing the space $\mathfrak{M}_{n}(\mathbb{C})$ by the subspaces of all symmetric or all antisymmetric matrices from $\mathfrak{M}_{n}(\mathbb{C})$ we obtain the following decompositions of symmetric powers of the irreducible representations of $G L(n, \mathbb{C})$ with highest weights $(2,0, \ldots, 0)$ and $(1,1,0, \ldots, 0)$, respectively:

$$
\begin{aligned}
& \sigma_{m}\left(T_{(2,0, \ldots, 0)}\right)=\sum_{\mathbf{m}} T_{\left(2 m_{1}, 2 m_{2}, \ldots, 2 m_{n}\right)}, \\
& \sigma_{m}\left(T_{(1,1,0, \ldots, 0)}\right)=\sum_{\mathbf{m}} T_{\left(m_{1}, m_{1}, \ldots, m_{k}, m_{k}\right)},
\end{aligned}
$$

where the summations are over those $\mathbf{m}=\left(m_{1}, m_{2}, \ldots, m_{n}\right)$ for which $m_{1} \geq m_{2} \geq \cdots \geq m_{n} \geq 0$ and $m_{1}+m_{2}+\cdots+m_{n}=m$. In the second formula $n=2 k$; if $n=2 k+1$, then on the right hand side we have to replace $T_{\left(m_{1}, m_{1}, \ldots, m_{k}, m_{k}\right)}$ by $T_{\left(m_{1}, m_{1}, \ldots, m_{k}, m_{k}, 0\right)}$.

\subsection{Properties of antisymmetric orbit functions of $A_{n}$}

We represent antisymmetric orbit functions of $A_{n}$ in the orthogonal coordinate system as in formula (4.9). Let $\lambda=\left(m_{1}, m_{2}, \ldots, m_{n+1}\right)$ and $\lambda+r=\left(m_{1}+r, m_{2}+r, \ldots, m_{n+1}+r\right)$, where $r$ is a fixed real number. If $x=\left(x_{1}, x_{2}, \ldots, x_{n+1}\right), x_{1}+x_{2}+\cdots+x_{n+1}=0$, and $w \in W$, then we have

$$
e^{2 \pi \mathrm{i}\langle\lambda+r, w x\rangle}=e^{2 \pi \mathrm{i}\langle\lambda, w x\rangle} e^{2 \pi \mathrm{i}\langle 0+r, w x\rangle}=e^{2 \pi \mathrm{i}\langle\lambda, w x\rangle} .
$$

It follows from this equality that

$$
\varphi_{\lambda}(x)=\varphi_{\lambda+r}(x), \quad \phi_{\lambda}(x)=\phi_{\lambda+r}(x)
$$

where $\lambda=\left(m_{1}, m_{2}, \ldots, m_{n+1}\right)$ is given in the orthogonal coordinate system. This means that instead of $m_{i}, i=1,2, \ldots, n+1$, determined by formulas of Subsection 3.3, we may assume that $m_{1}, m_{2}, \ldots, m_{n+1}$ are integers such that $m_{1} \geq m_{2} \geq \cdots \geq m_{n+1} \geq 0$. We adopt this assumption in this subsection.

For simplicity we introduce the following notations:

$$
e^{2 \pi \mathrm{i} x_{j}}=y_{j}, \quad j=1,2, \ldots, n+1 .
$$


In order to receive relations for antisymmetric orbit functions we have to take characters of representations on both sides of relations of the previous subsection and to substitute the expression

$$
\chi_{\lambda}(x)=\frac{\varphi_{\lambda+\rho}(x)}{\varphi_{\rho}(x)}
$$

for characters (see Subsection 9.1 below).

The relation (6.4) gives the equality

$$
\chi_{\sigma_{m}(T)}\left(g_{1}, g_{2}\right)=\sum_{\sum_{i} m_{i}=m} \chi_{s}\left(g_{1}\right) \chi_{s^{\prime}}\left(g_{2}\right)
$$

for characters of representations, where $s^{\prime}=\left(s_{1}, s_{2}, \ldots, s_{n_{1}}, 0, \ldots, 0\right)$ and the sum is such as in (6.4). Multiply both sides by $t^{m}$ and sum up over all non-negative integral values of $m$. We get

$$
\sum_{m=0}^{\infty} t^{m} \chi_{\sigma_{m}(T)}\left(g_{1}, g_{2}\right)=\sum_{m=0}^{\infty} t^{m} \sum_{\sum_{i} m_{i}=m} \chi_{s}\left(g_{1}\right) \chi_{s^{\prime}}\left(g_{2}\right) .
$$

Take this relation for $g_{1}=\operatorname{diag}\left(y_{1}, y_{2}, \ldots, y_{n_{1}}\right)$ and $g_{2}=\operatorname{diag}\left(z_{1}, z_{2}, \ldots, z_{n_{2}}\right)$. For such $g_{1}$ and $g_{2}$ the left hand side takes the form

$$
\sum_{m=0}^{\infty} t^{m} \chi_{\sigma_{m}(T)}\left(g_{1}, g_{2}\right)=\sum_{m=0}^{\infty} t^{m} \sum y_{1}^{r_{1}} y_{2}^{r_{2}} \cdots y_{n_{1}}^{r_{n_{1}}} z_{1}^{p_{1}} z_{2}^{p_{2}} \cdots z_{n_{2}}^{p_{n_{2}}},
$$

where the second summation on the right hand side is over integral $r_{i}$ and $p_{j}$ such that $\sum_{i=1}^{n_{1}} r_{i}+$ $\sum_{j=1}^{n_{2}} p_{j}=m$. Therefore, the relation (6.13) can be written as

$$
\sum_{m=0}^{\infty} t^{m} \sum_{\sum_{i} m_{i}=m} \chi_{s}\left(g_{1}\right) \chi_{s^{\prime}}\left(g_{2}\right)=\prod_{i=1}^{n_{1}} \prod_{j=1}^{n_{2}}\left(1-t y_{i} z_{j}\right)^{-1}
$$

where $g_{1}=\operatorname{diag}\left(y_{1}, y_{2}, \ldots, y_{n_{1}}\right), g_{2}=\operatorname{diag}\left(z_{1}, z_{2}, \ldots, z_{n_{2}}\right)$ and $|t|<1,\left|y_{i}\right|<1,\left|z_{j}\right|<1$.

Further, we represent characters in (6.13) in terms of antisymmetric orbit functions and set $t=1$. As a result, we receive the following relation for antisymmetric orbit functions:

$$
\varphi_{\rho_{1}}^{\left(n_{1}\right)}(y) \varphi_{\rho_{2}}^{\left(n_{2}\right)}(z) \prod_{i=1}^{n_{1}} \prod_{j=1}^{n_{2}}\left(1-y_{i} z_{j}\right)^{-1}=\sum_{m} \varphi_{s+\rho_{1}}^{\left(n_{1}\right)}(y) \varphi_{\hat{s}+\rho_{2}}^{\left(n_{2}\right)}(z),
$$

where $y=\left(y_{1}, y_{2}, \ldots, y_{n_{1}}\right), z=\left(z_{1}, z_{2}, \ldots, z_{n_{2}}\right)$, and $\rho_{1}$ and $\rho_{2}$ are half-sums of positive roots for $A_{n_{1}-1}$ and $A_{n_{2}-1}$, respectively. In particular, if $n_{1}=n_{2}=n$, then

$$
\varphi_{\rho}(y) \varphi_{\rho}(z) \prod_{i, j=1}^{n}\left(1-y_{i} z_{j}\right)^{-1}=\sum_{m} \varphi_{s+\rho}(y) \varphi_{s+\rho}(z) .
$$

Applying to this relation the Cauchy lemma, which states that

$$
\operatorname{det}\left(\left(1-y_{i} z_{j}\right)^{-1}\right)_{i, j=1}^{n}=\varphi_{\rho}(y) \varphi_{\rho}(z) \prod_{i, j=1}^{n}\left(1-y_{i} z_{j}\right)^{-1}
$$


we obtain the identity

$$
\sum_{\lambda} \varphi_{\lambda+\rho}(y) \varphi_{\lambda+\rho}(z)=\operatorname{det}\left(\left(1-y_{i} z_{j}\right)^{-1}\right)_{i, j=1}^{n} .
$$

Now we use the relation (6.10) for representations of the group $G L(n, \mathbb{C})$ in order to derive the equality

$$
\chi_{(2,0, \ldots, 0), m}(g)=\sum_{|\mathbf{m}|=m} \chi_{2 \mathbf{m}}(g), \quad g \in G L(n, \mathbb{C}),
$$

where on the left hand side is the character of the representation $\sigma_{m}\left(T_{(2,0, \ldots, 0)}\right)$ from $(6.10)$, $2 \mathbf{m}=\left(2 m_{1}, 2 m_{2}, \ldots, 2 m_{n}\right), m_{1} \geq m_{2} \geq \cdots \geq m_{n} \geq 0,|\mathbf{m}|=m_{1}+m_{2}+\cdots+m_{n}$. We derive from this equality that

$$
\sum_{m=0}^{\infty} \chi_{(2,0, \ldots, 0), m}(g) t^{m}=\sum_{m=0}^{\infty} t^{m} \sum_{|\mathbf{m}|=m} \chi_{2 \mathbf{m}}(g) .
$$

Setting $g=\operatorname{diag}\left(y_{1}, y_{2}, \ldots, y_{n}\right)$, for the left hand side we have the expression

$$
\sum_{m=0}^{\infty} \chi_{(2,0, \ldots, 0), m}(g) t^{m}=\prod_{1 \leq i \leq j \leq n}\left(1-t y_{i} y_{j}\right)^{-1}
$$

where $\left|y_{i}\right|<1$ and $|t|<1$. Now using the Weyl character formula one receives

$$
\sum_{\mathbf{m}} \varphi_{2 \mathbf{m}+\rho}(y) t^{|\mathbf{m}|}=\varphi_{\rho}(y) \prod_{1 \leq i \leq j \leq n}\left(1-t y_{i} y_{j}\right)^{-1}
$$

Substituting into (6.16) the expression for $\varphi_{2 \mathbf{m}+\rho}(y)$, setting $t=1$ and using the evident relation

$$
\sum_{\mathbf{m}} a_{1}^{m_{1}} a_{2}^{m_{2}} \cdots a_{n}^{m_{n}}=\left(1-a_{1}\right)^{-1}\left(1-a_{1} a_{2}\right)^{-1} \cdots\left(1-a_{1} a_{2} \cdots a_{n}\right)^{-1},
$$

we get a non-trivial identity

$$
\sum_{\left(i_{1}, i_{2}, \ldots, i_{n}\right)} \frac{\operatorname{sign}\left(i_{1}, i_{2}, \ldots, i_{n}\right) y_{i_{1}}^{n-1} y_{i_{2}}^{n-2} \cdots y_{i_{n-1}}}{\left(1-y_{i_{1}}^{2}\right)\left(1-y_{i_{1}}^{2} y_{i_{2}}^{2}\right) \cdots\left(1-y_{i_{1}}^{2} \cdots y_{i_{n}}^{2}\right)}=\varphi_{\rho}(y) \prod_{1 \leq i \leq j \leq n}\left(1-y_{i} y_{j}\right)^{-1},
$$

where summation is over all permutations $\left(i_{1}, i_{2}, \ldots, i_{n}\right)$ of the natural numbers $1,2, \ldots, n$ and $\operatorname{sign}\left(i_{1}, i_{2}, \ldots, i_{n}\right)$ means a sign of the permutation $\left(i_{1}, i_{2}, \ldots, i_{n}\right)$.

In the same way, from the decomposition (6.11) we derive

$$
\sum_{\mathbf{m}} \varphi_{\tilde{\mathbf{m}}+\rho}(y) t^{|\mathbf{m}|}=\varphi_{\rho}(y) \prod_{1 \leq i<j \leq n}\left(1-t y_{i} y_{j}\right)^{-1}
$$

where summation is over $\mathbf{m}=\left(m_{1}, m_{2}, \ldots, m_{k}\right), k=[n / 2], m_{1} \geq m_{2} \geq \cdots \geq m_{k} \geq 0$, $\tilde{\mathbf{m}}=\left(m_{1}, m_{1}, m_{2}, m_{2}, \ldots, m_{k}, m_{k}\right)$ (here one has to add 0 at the end if $\left.n=2 k+1\right)$. As above, we receive from here the identity

$$
\sum_{\left(i_{1}, i_{2}, \ldots, i_{n}\right)} \frac{\operatorname{sign}\left(i_{1}, i_{2}, \ldots, i_{n}\right) y_{i_{1}}^{n-1} y_{i_{2}}^{n-2} \cdots y_{i_{n-1}}}{\left(1-y_{i_{1}} y_{i_{2}}\right)\left(1-y_{i_{1}} y_{i_{2}} y_{i_{3}} y_{i_{4}}\right) \cdots\left(1-y_{i_{1}} \cdots y_{i_{2 \nu}}\right)}=\varphi_{\rho}(y) \prod_{1 \leq i \leq j \leq n}\left(1-y_{i} y_{j}\right)^{-1} .
$$


In order to derive other identities we note that for $\theta=\left(\theta_{1}, \theta_{2}, \ldots, \theta_{n}\right)$ one has

$$
\lim _{\theta \rightarrow 0} \varphi_{\lambda+\rho}\left(e^{2 \pi \mathrm{i} \theta}\right)=\varphi_{\rho}(\lambda+\rho) .
$$

Taking into account this relation and substituting $y=(1, \ldots, 1)$ and $z=(1, \ldots, 1)$ into $(6.14)$, we derive a relation for the antisymmetric orbit functions $\varphi_{\rho}$ :

$$
\sum_{|\mathbf{m}|=m} \varphi_{\rho_{s}}^{(s)}\left(\mathbf{m}+\rho_{s}\right) \varphi_{\rho_{r}}^{(r)}\left(\hat{\mathbf{m}}+\rho_{r}\right)=\frac{(s r+m-1) !}{(s r-1) ! m !} \varphi_{\rho_{s}}^{(s)}\left(\rho_{s}\right) \varphi_{\rho_{r}}^{(r)}\left(\rho_{r}\right),
$$

where $r \geq s \geq 0, \mathbf{m}=\left(m_{1}, m_{2}, \ldots, m_{s}\right), \hat{\mathbf{m}}=\left(m_{1}, m_{2}, \ldots, m_{s}, 0, \ldots, 0\right),|\mathbf{m}|=m_{1}+m_{2}+\cdots+$ $m_{s}$ and $\rho_{k}=(k-1, k-2, \ldots, 1,0)$.

From the relations (6.16) and (6.17) one similarly obtains the identities

$$
\begin{aligned}
& \sum_{|\mathbf{m}|} \varphi_{\rho}(2 \mathbf{m}+\rho)=\frac{\left(m+\frac{1}{2} n(n+1)-1\right) !}{\left(\frac{1}{2} n(n+1)-1\right) ! m !} \varphi_{\rho}(\rho), \\
& \sum_{|\mathbf{m}|} \varphi_{\rho}(\tilde{\mathbf{m}}+\rho)=\frac{\left(m+\frac{1}{2} n(n-1)-1\right) !}{\left(\frac{1}{2} n(n-1)-1\right) ! m !} \varphi_{\rho}(\rho),
\end{aligned}
$$

where $\mathbf{m}=\left(m_{1}, m_{2}, \ldots, m_{\nu}\right), \tilde{\mathbf{m}}=\left(m_{1}, m_{1}, m_{2}, m_{2}, \ldots, m_{\nu}, m_{\nu}\right), \nu=[n / 2]$.

From (6.18)-(6.20) we derive the relations

$$
\begin{aligned}
& \sum_{\mathbf{m}} \varphi_{\rho_{s}}^{(s)}\left(\mathbf{m}+\rho_{s}\right) \varphi_{\rho_{r}}^{(r)}\left(\hat{\mathbf{m}}+\rho_{r}\right) t^{|\mathbf{m}|}=(1-t)^{-s r} \varphi_{\rho_{s}}^{(s)}\left(\rho_{s}\right) \varphi_{\rho_{r}}^{(r)}\left(\rho_{r}\right), \\
& \sum_{\mathbf{m}} \varphi_{\rho}(2 \mathbf{m}+\rho) t^{|\mathbf{m}|}=(1-t)^{-n(n+1) / 2} \varphi_{\rho}(\rho), \\
& \sum_{\mathbf{m}} \varphi_{\rho}(2 \tilde{\mathbf{m}}+\rho) t^{|\mathbf{m}|}=(1-t)^{-n(n-1) / 2} \varphi_{\rho}(\rho) .
\end{aligned}
$$

Other decompositions of the previous subsection lead to new relations for antisymmetric orbit functions of $A_{n}$.

\section{Decomposition of products of (anti)symmetric orbit functions}

The aim of this section is to derive how to decompose products of (anti)symmetric orbit functions into sums of (anti)symmetric orbit functions. Such operations are fulfilled by means of the corresponding decompositions of (signed) orbits.

\subsection{Products of symmetric and antisymmetric orbit functions}

Invariance of symmetric orbit functions $\phi_{\lambda}$ and anti-invariance of antisymmetric orbit functions $\varphi_{\lambda}$ with respect to the Weyl group $W$ lead to the following statements:

Proposition 11. (a) A product of symmetric orbit functions expands into a sum of symmetric orbit functions:

$$
\phi_{\lambda} \phi_{\mu}=\sum_{\nu} n_{\nu} \phi_{\nu}
$$

where an integer $n_{\nu}$ shows how many times the orbit function $\phi_{\nu}$ is contained in the product $\phi_{\lambda} \phi_{\mu}$. 
(b) A product of antisymmetric orbit functions expands into a sum of symmetric orbit functions:

$$
\varphi_{\lambda} \varphi_{\mu}=\sum_{\nu} n_{\nu} \phi_{\nu}
$$

where $n_{\nu}$ are positive or negative integral coefficients.

(c) A product of symmetric and antisymmetric orbit functions expands into a sum of antisymmetric orbit functions:

$$
\phi_{\lambda} \varphi_{\mu}=\sum_{\nu} n_{\nu} \varphi_{\nu}
$$

where $n_{\nu}$ are positive or negative integral coefficients.

Proof. In the case (b) we have

$$
\varphi_{\lambda}(w x) \varphi_{\mu}(w x)=(\operatorname{det} w)^{2} \varphi_{\lambda}(x) \varphi_{\mu}(x)=\varphi_{\lambda}(x) \varphi_{\mu}(x) .
$$

Therefore, the product $\varphi_{\lambda} \varphi_{\mu}$ is invariant with respect to $W$. Hence, it can be expanded into symmetric orbit functions (see Subsection 7.8 in [1]). Since antisymmetric orbit functions $\varphi_{\lambda}$ are sums of exponential functions $e^{2 \pi \mathrm{i}\langle\sigma, x\rangle}$ with coefficients \pm 1 , the coefficients $n_{\nu}$ in (7.2) are integers. In the case (c) we have a similar situation. The case (a) is considered in [1].

In order to fulfill expansions (a)-(c) in an explicit form it is necessary to fulfill the corresponding decompositions of products of (signed) orbit, considering usual (not signed) orbits as signed orbits in which to all points the sign "+" is assigned, and to take into account that multiplication of (singed) orbit functions are reduced to multiplication of exponential functions.

A product $O^{ \pm}(\lambda) \otimes O^{ \pm}\left(\lambda^{\prime}\right)$ of two signed orbits $O^{ \pm}(\lambda)$ and $O^{ \pm}\left(\lambda^{\prime}\right)$ (one or two of them can be replaced by usual orbits, that is, to the corresponding points the sign "+" is assigned) is the set of all points of the form $\lambda_{1}+\lambda_{2}$ (where $\lambda_{1} \in O^{ \pm}(\lambda)$ and $\lambda_{2} \in O^{ \pm}\left(\lambda^{\prime}\right)$ ) with a sign which is a product of signs of $\lambda$ and $\lambda^{\prime}$. Since a set of points $\lambda_{1}+\lambda_{2}$ (without signs), $\lambda_{1} \in O(\lambda), \lambda_{2} \in O\left(\lambda^{\prime}\right)$, is invariant with respect to action of the corresponding Weyl group, each product of orbits is decomposable into a sum of orbits. Then it follows from assertions (a)-(c) of Proposition 11 that, considering points $\lambda_{1}+\lambda_{2}$ with signs, we obtain decomposition of $O^{ \pm}(\lambda) \otimes O^{ \pm}\left(\lambda^{\prime}\right)$ into usual orbits, where to each point a sign is assigned (the same sign for points of a fixed orbit). Moreover, a product of a signed orbit with a usual orbit decomposes into signed orbits (not into usual orbits).

Under product of (signed) orbits we may receive an orbit with signed points in which the sign "-" corresponds to a dominant weight. This means that we obtain a signed orbit with opposite signs for its points. In this case we say that a product of (signed) orbits contains the corresponding signed orbit with sign "-" and denote it by $-O^{ \pm}(\mu)$. That is why in (7.2) and (7.3) negative coefficients can appear.

Example. Orbits of $A_{1}$. If $a \in E_{1}$ is strictly positive, then the signed orbit of this point $O^{ \pm}(a)$ consists of two signed points $a^{+}$and $-a^{-}$. It is easy to see that for the product $O^{ \pm}(a) \otimes O^{ \pm}(b)$ we have

$$
\begin{aligned}
O^{ \pm}(a) \otimes O^{ \pm}(b) & \equiv\left\{a^{+},-a^{-}\right\} \otimes\left\{b^{+},-b^{-}\right\} \\
& =\left\{(a+b)^{+},(-a-b)^{+}\right\} \cup\left\{(|a-b|)^{-},(-|a-b|)^{-}\right\} \\
& =O(a+b) \cup-O(|a-b|),
\end{aligned}
$$

where $O(a+b)$ and $O(|a-b|)$ are usual (not signed) orbits. Therefore,

$$
\varphi_{a}(x) \varphi_{b}(x)=\phi_{a+b}(x)-\phi_{|a-b|}(x) .
$$


Similarly, we have

$$
\begin{aligned}
& O^{ \pm}(a) \otimes O(b)=O^{ \pm}(a+b) \cup O^{ \pm}(a-b), \quad a>b>0, \\
& O^{ \pm}(a) \otimes O(b)=O^{ \pm}(a+b) \cup-O^{ \pm}(b-a), \quad b>a>0 .
\end{aligned}
$$

Thus,

$$
\begin{aligned}
& \varphi_{a}(x) \varphi_{b}(x)=\varphi_{a+b}(x)+\varphi_{a-b}(x), \quad a>b>0 \\
& \varphi_{a}(x) \phi_{b}(x)=\varphi_{a+b}(x)-\varphi_{|a-b|}(x), \quad b>a>0 .
\end{aligned}
$$

For the corresponding decompositions for $O(a) \otimes O(b)$ see [1].

Decomposition of products of orbits in higher dimension of the Euclidean space is not a simple task. In the next subsection we consider some general results on the decomposition.

\subsection{Products of symmetric and antisymmetric orbits}

Let $O(\lambda)=\left\{w \lambda \mid w \in W / W_{\lambda}\right\}$ be a usual orbit and $O^{ \pm}(\mu)=\{w \mu \mid w \in W\}$ be a signed orbit. Then

$$
\begin{aligned}
O(\lambda) \otimes O^{ \pm}(\mu)= & \left\{\left(w \lambda+w^{\prime} \mu\right)^{\operatorname{det} w^{\prime}} \mid w \in W / W_{\lambda}, w^{\prime} \in W\right\} \\
= & \left\{\left(w \lambda+w_{1} \mu\right)^{\operatorname{det} w_{1}} \mid w \in W / W_{\lambda}\right\} \cup\left\{\left(w \lambda+w_{2} \mu\right)^{\operatorname{det} w_{2}} \mid w \in W / W_{\lambda}\right\} \cup \cdots \\
& \cup\left\{\left(w \lambda+w_{s} \mu\right)^{\operatorname{det} w_{s}} \mid w \in W / W_{\lambda}\right\},
\end{aligned}
$$

where $w_{1}, w_{2}, \ldots, w_{s}$ is the set of elements of $W$. Since a product of an orbit and a signed orbit decomposes into signed orbits, for decomposition of the product $O(\lambda) \otimes O^{ \pm}(\mu)$ into separate signed orbits it is necessary to take dominant elements from each term of the right hand side of (7.4). That is, $O(\lambda) \otimes O^{ \pm}(\mu)$ is a union of the signed orbits, corresponding to points from

$$
\begin{aligned}
& D\left(\left\{\left(w \lambda+w_{1} \mu\right)^{\operatorname{det} w_{1}} \mid w \in W / W_{\lambda}\right\}\right), \quad D\left(\left\{\left(w \lambda+w_{2} \mu\right)^{\operatorname{det} w_{2}} \mid w \in W / W_{\lambda}\right\}\right), \quad \ldots, \\
& D\left(\left\{\left(w \lambda+w_{s} \mu\right)^{\operatorname{det} w_{s}} \mid w \in W / W_{\lambda}\right\}\right),
\end{aligned}
$$

where $D\left(\left\{\left(w \lambda+w_{i} \mu\right)^{\operatorname{det} w_{i}} \mid w \in W / W_{\lambda}\right\}\right)$ means the set of dominant signed elements in $\{(w \lambda+$ $\left.\left.w_{i} \mu\right)^{\operatorname{det} w_{i}} \mid w \in W / W_{\lambda}\right\}$.

Proposition 12. The product $O(\lambda) \otimes O^{ \pm}(\mu)$ consists only of signed orbits of the form $O^{ \pm}(\mid w \lambda+$ $\mu \mid), w \in W / W_{\lambda}$, where $|w \lambda+\mu|$ is a dominant weight of the orbit containing $w \lambda+\mu$. Moreover, each such orbit $O^{ \pm}(|w \lambda+\mu|), w \in W / W_{\lambda}$, except for those of them, for which $|w \lambda+\mu|$ lies on some wall of the dominant Weyl chamber, belongs to the product $O(\lambda) \otimes O^{ \pm}(\mu)$.

Proof. For each dominant element $w \lambda+w_{i} \mu$ from (7.5) there exists an element $w^{\prime \prime} \in W$ such that $w^{\prime \prime}\left(w \lambda+w_{i} \mu\right)=w^{\prime} \lambda+\mu$. It means that $w \lambda+w_{i} \mu$ is of the form $\left|w^{\prime} \lambda+\mu\right|, w^{\prime} \in W / W_{\lambda}$. It is clear that a sign of this dominant element is $\operatorname{det} w_{i} w^{\prime \prime}$. Conversely, take any element $w \lambda+\mu$, $w \in W / W_{\lambda}$. It belongs (with some sign) to the product $O(\lambda) \otimes O^{ \pm}(\mu)$. This means that $|w \lambda+\mu|$ also belongs to this product. Therefore, the signed orbit $O^{ \pm}(|w \lambda+\mu|)$ is contained in $O(\lambda) \otimes O^{ \pm}(\mu)$ with some coefficient if $|w \lambda+\mu|$ does not lie on some wall of the dominant Weyl chamber. This coefficient cannot vanish since if it vanishes, then in the product $O(\lambda) \otimes O^{ \pm}(\mu)$ there are contained points of $O^{ \pm}(|w \lambda+\mu|)$, but taken with an opposite signs. In this case there exists another element $w^{\prime} \lambda+\mu$ such that $w \lambda+\mu=w^{\prime \prime}\left(w^{\prime} \lambda+\mu\right)$. Since $\mu$ does not lie on a wall, this is not possible. Proposition is proved. 
It follows from Proposition 12 that for decomposition of the product $O(\lambda) \otimes O^{ \pm}(\mu)$ into separate signed orbits we have to take all elements $w \lambda+\mu, w \in W / W_{\lambda}$, and to find the corresponding strictly dominant elements $|w \lambda+\mu|, w \in W / W_{\lambda}$.

Corollary. For the product $O(\lambda) \otimes O^{ \pm}(\mu)$ we have

$$
O(\lambda) \otimes O^{ \pm}(\mu)=\bigcup_{w \in W / W_{\lambda}}^{\prime} \varepsilon_{w} O^{ \pm}(|w \lambda+\mu|),
$$

where the prime means that the term with $|w \lambda+\mu|$ lying on a wall must be omitted, and $\varepsilon_{w}$ is equal to +1 or -1 .

Proof. This corollary is similar to Proposition 4 in [1]. A proof is also similar. We only have to take into account that signed orbits can be contained in $O(\lambda) \otimes O^{ \pm}(\mu)$ with the sign "-". The corollary is proved.

Note that in the case of product $O(\lambda) \otimes O(\mu), \lambda \in P_{+}, \mu \in P_{+}$, of usual orbits the orbits $O(\nu)$ with multiplicities $m_{\nu}>1$ may appear in the decomposition . As we see from Corollary, all coefficients in the decomposition (7.6) are modulo 1 . The only problem which appears here is to find signs of the coefficients $\varepsilon_{\nu}$.

According to Corollary we have

$$
\phi_{\lambda}(x) \varphi_{\mu}(x)=\sum_{w \in W / W_{\lambda}}^{\prime} \varepsilon_{w} \varphi_{|w \lambda+\mu|}(x),
$$

where summation is such as in (7.6) and $\varepsilon_{w}$ are equal to +1 or -1 .

Proposition 13. Let $O(\lambda), \lambda \neq 0$, be an orbit and let $O^{ \pm}(\mu)$ be a signed orbit. If all elements $w \lambda+\mu, w \in W / W_{\lambda}$, are dominant, then

$$
O(\lambda) \otimes O^{ \pm}(\mu)=\bigcup_{w \in W / W_{\lambda}}^{\prime} O^{ \pm}(w \lambda+\mu),
$$

where the prime means that terms corresponding to $w \lambda+\mu$ lying on a wall must be omitted.

Proof. The statement of this proposition follows from the above corollary.

At the end of this subsection we formulate the following method for decomposition of products $O(\lambda) \otimes O^{ \pm}(\mu)$, which follows from statement of Proposition 12. On the first step we shift all points of the orbit $O(\lambda)$ by $\mu$. As a result, we obtain the set of points $w \lambda+\mu, w \in W / W_{\lambda}$. On the second step, we map non-dominant elements of this set by elements of the Weyl group $W$ to the dominant Weyl chamber. On this step we obtain the set $|w \lambda+\mu|, w \in W / W_{\lambda}$. Then according to Proposition 12, $O(\lambda) \otimes O^{ \pm}(\mu)$ consists of the signed orbits $O^{ \pm}(|w \lambda+\mu|)$ for which $|w \lambda+\mu|$ do not lie on a wall of the dominant Weyl chamber. On the third step, we determine signs of these orbits, taking into account the above propositions or making a direct calculation.

\subsection{Products of antisymmetric orbits}

Let $O^{ \pm}(\lambda)=\left\{(w \lambda)^{\operatorname{det} w} \mid w \in W\right\}$ and $O^{ \pm}(\mu)=\left\{(w \mu)^{\operatorname{det} w} \mid w \in W\right\}$ be two signed orbits, where $\lambda$ and $\mu$ are strictly dominant elements of $E_{n}$. Then

$$
\begin{aligned}
O^{ \pm}(\lambda) \otimes O^{ \pm}(\mu)= & \left\{\left(w \lambda+w^{\prime} \mu\right)^{\operatorname{det} w w^{\prime}} \mid w \in W, w^{\prime} \in W\right\} \\
= & \left\{\left(w \lambda+w_{1} \mu\right)^{\operatorname{det} w w_{1}} \mid w \in W\right\} \cup\left\{\left(w \lambda+w_{2} \mu\right)^{\operatorname{det} w w_{2}} \mid w \in W\right\} \cup \cdots \\
& \cup\left\{\left(w \lambda+w_{s} \mu\right)^{\operatorname{det} w w_{s}} \mid w \in W\right\},
\end{aligned}
$$


where $w_{1}, w_{2}, \ldots, w_{s}$ is the set of all elements of $W$. Since a product of two signed orbits decomposes into usual orbits (all points of some of these orbits are taken with the sign "-"), for decomposition of the product $O^{ \pm}(\lambda) \otimes O^{ \pm}(\mu)$ into separate orbits it is necessary to take dominant elements from each term of the right hand side of (7.7). That is, $O^{ \pm}(\lambda) \otimes O^{ \pm}(\mu)$ is a union of the orbits (some of them with the sign "-") corresponding to points from

$$
\begin{aligned}
& D\left(\left\{\left(w \lambda+w_{1} \mu\right)^{\operatorname{det} w w_{1}} \mid w \in W\right\}\right), \quad D\left(\left\{\left(w \lambda+w_{2} \mu\right)^{\operatorname{det} w w_{2}} \mid w \in W\right\}\right), \quad \ldots, \\
& D\left(\left\{\left(w \lambda+w_{s} \mu\right)^{\operatorname{det} w w_{s}} \mid w \in W\right\}\right),
\end{aligned}
$$

where $D\left(\left\{\left(w \lambda+w_{i} \mu\right)^{\operatorname{det} w w_{i}} \mid w \in W\right\}\right)$ means the set of dominant signed elements in $\{(w \lambda+$ $\left.\left.w_{i} \mu\right)^{\operatorname{det} w w_{i}} \mid w \in W\right\}$.

Proposition 14. The product $O^{ \pm}(\lambda) \otimes O^{ \pm}(\mu)$ consists only of orbits (with signs "+ " or "- ") of the form $O(|w \lambda+\mu|), w \in W$, where $|w \lambda+\mu|$ is a dominant weight of the orbit containing $w \lambda+\mu$. Moreover, each such orbit $O(|w \lambda+\mu|), w \in W$, for which $|w \lambda+\mu|$ does not lie on some wall of the dominant Weyl chamber, belongs to the product $O^{ \pm}(\lambda) \otimes O^{ \pm}(\mu)$.

Proof. For each dominant element $w \lambda+w_{i} \mu$ from (7.8) there exists an element $w^{\prime \prime} \in W$ such that $w^{\prime \prime}\left(w \lambda+w_{i} \mu\right)=w^{\prime} \lambda+\mu$. It means that $w \lambda+w_{i} \mu$ is of the form $\left|w^{\prime} \lambda+\mu\right|, w^{\prime} \in W$, and the first part of the proposition follows.

Take any element $w \lambda+\mu, w \in W$, which does not lie on a wall. Then $|w \lambda+\mu|$ does not lie on a wall. Then $w \lambda+\mu$ belongs (with some sign) to the product $O^{ \pm}(\lambda) \otimes O^{ \pm}(\mu)$. This means that $|w \lambda+\mu|$ also belongs to this product. Therefore, the orbit $O(|w \lambda+\mu|)$ is contained in $O^{ \pm}(\lambda) \otimes O^{ \pm}(\mu)$ with some coefficient. This coefficient cannot vanish since if it vanishes, then in the product $O^{ \pm}(\lambda) \otimes O^{ \pm}(\mu)$ there are contained points of $O^{ \pm}(|w \lambda+\mu|)$, but taken with an opposite signs. In this case there exists another element $w^{\prime} \lambda+\mu$ such that $w \lambda+\mu=w^{\prime \prime}\left(w^{\prime} \lambda+\mu\right)$. Since $\mu$ does not lie on a wall, this is not possible. Proposition is proved.

It follows from Proposition 14 that for decomposition of the product $O^{ \pm}(\lambda) \otimes O^{ \pm}(\mu)$ into orbits we have to take all elements $w \lambda+\mu, w \in W$, and to find the corresponding dominant elements $|w \lambda+\mu|, w \in W$.

Corollary. For the product $O^{ \pm}(\lambda) \otimes O^{ \pm}(\mu)$ we have

$$
O^{ \pm}(\lambda) \otimes O^{ \pm}(\mu)=\bigcup_{w \in W} \varepsilon_{w} O(|w \lambda+\mu|)
$$

where $\varepsilon_{w}$ is equal to +1 or -1 if $|w \lambda+\mu|$ does not lie on some wall.

In the case of product $O^{ \pm}(\lambda) \otimes O^{ \pm}(\mu), \lambda \in P_{+}^{+}, \mu \in P_{+}^{+}$, of sighed orbits in the decomposition may appear orbits $O(\nu)$ with integral coefficients $m_{\nu}$ such that $m_{\nu}>1$. Such coefficients may appear only for $\nu$ lying on a wall.

Proposition 15. Let $O^{ \pm}(\lambda)$ and $O^{ \pm}(\mu)$ be two signed orbits. If all elements $w \lambda+\mu, w \in W$ are dominant, then

$$
O^{ \pm}(\lambda) \otimes O^{ \pm}(\mu)=\bigcup_{w \in W} \varepsilon_{w} O(w \lambda+\mu)
$$

where $\varepsilon_{w}$ is equal to +1 or -1 , if $|w \lambda+\mu|$ does not lie on some wall.

This proposition is proved in the same way as Proposition 14 and we omit it. 
Proposition 16. Let $O^{ \pm}(\lambda)$ and $O^{ \pm}(\mu)$ be sighed orbits, and let all elements $w \lambda+\mu, w \in W$, be strictly dominant (that is, they are dominant and do not belong to any wall of the dominant Weyl chamber). Then

$$
O^{ \pm}(\lambda) \otimes O^{ \pm}(\mu)=\bigcup_{w \in W} \varepsilon_{w} O(w \lambda+\mu)
$$

where $\varepsilon_{w}$ are equal to +1 or -1 .

This proposition is proved in the same way as Proposition 2 in [1].

\subsection{Decomposition of products for rank 2}

We give here examples of decompositions of products of (signed) orbits for the cases $A_{2}$ and $C_{2}$. Orbits for these cases are placed on the plane. Therefore, decompositions can be done by geometrical calculations on this plane. These cases can be easily considered by using for orbit points the orthogonal coordinates from Section 3. The corresponding Weyl groups have a simple description in these coordinates and this gives a possibility to make calculations in a simple manner.

For the case of $A_{2}$ at $a \neq b$ we have

$$
\begin{aligned}
& A_{2}: O^{ \pm}(a b) \otimes O(c 0)=O^{ \pm}(a+c b) \cup O^{ \pm}(a-c b+c) \\
& \cup-O^{ \pm}(a+b-c c-b) \quad(a>c>b), \\
& O^{ \pm}(a b) \otimes O(c 0)=O^{ \pm}(a+c b) \cup O^{ \pm}(a-c b+c) \\
& \cup O^{ \pm}(a b-c) \quad(a>c, b>c), \\
& O^{ \pm}(a b) \otimes O(c 0)=O^{ \pm}(a+c b) \quad(a=b=c), \\
& \left.O^{ \pm}(a b) \otimes O(c 0)=O^{ \pm}(a+c b) \cup-O^{ \pm}(a+b-c c-b)\right) \quad(a=c>b), \\
& O^{ \pm}(a b) \otimes O(c 0)=O^{ \pm}(a+c b) \cup O^{ \pm}(a b-c) \quad(b>a=c), \\
& O^{ \pm}(a b) \otimes O(c 0)=O^{ \pm}(a+c b) \cup-O^{ \pm}(c-a a+b) \quad(a<b=c) \text {, } \\
& O^{ \pm}(a b) \otimes O(c 0)=O^{ \pm}(a+c b) \cup-O^{ \pm}(c-a a+b) \\
& \cup O^{ \pm}(c-a-b a) \quad(c>a+b), \\
& O^{ \pm}(a b) \otimes O(c 0)=O^{ \pm}(a+c b) \cup-O^{ \pm}(c-a a+b) \\
& \cup-O^{ \pm}(a+b-c c-a) \quad(a+b>c>b), \\
& O^{ \pm}(a b) \otimes O(c 0)=O^{ \pm}(a+c b) \cup-O^{ \pm}(c-a a+b) \\
& \cup O^{ \pm}(a b-c) \quad(a+b>b>c) .
\end{aligned}
$$

If $a=b$, then we get

$$
\begin{aligned}
& O^{ \pm}(a a) \otimes O(c 0)=O^{ \pm}(a+c a) \cup O^{ \pm}(c-2 a a) \\
& \cup-O^{ \pm}(c-a 2 a) \quad(c>2 a), \\
& O^{ \pm}(a a) \otimes O(c 0)=O^{ \pm}(a+c a) \cup-O^{ \pm}(2 a-c c-a) \\
& \cup-O^{ \pm}(c-a 2 a) \quad(2 a>c>a), \\
& O^{ \pm}(a a) \otimes O(c 0)=O^{ \pm}(a+c a) \cup O^{ \pm}(a-c a+c) \\
& \cup O^{ \pm}(a a-c) \quad(a>c) .
\end{aligned}
$$

Similar products of $C_{2}$ orbits are of the form

$$
\begin{gathered}
C_{2}: \quad O^{ \pm}(a b) \otimes O(c 0)=O^{ \pm}(a+c b) \cup-O^{ \pm}(-a-2 b+c b) \cup-O^{ \pm}(c-a a+b) \\
\cup O^{ \pm}(c-2 b-a a+b) \quad(a+b-c<b),
\end{gathered}
$$




$$
\begin{gathered}
O^{ \pm}(a b) \otimes O(c 0)=O^{ \pm}(a+c b) \cup O^{ \pm}(a+2 b-c c-a-b) \cup O^{ \pm}(a-c b+c) \\
\cup-O^{ \pm}(a+2 b-c c-b) \quad(b>c-a-b, a>c), \\
\begin{aligned}
O^{ \pm}(a b) \otimes O(c 0)= & O^{ \pm}(a+c b) \cup O^{ \pm}(a+2 b-c c-a-b) \cup-O^{ \pm}(c-a a+b) \\
\cup-O^{ \pm}(a+2 b-c c-b) & (b>c-a-b, c>a), \\
O^{ \pm}(a b) \otimes O(c 0)= & O^{ \pm}(a+c b) \cup O^{ \pm}(a-c b) \cup O^{ \pm}(a-c b+c) \\
\cup-O^{ \pm}(a+2 b-c c-b) & (a+b>b+c), \\
O^{ \pm}(a b) \otimes O(c 0)=O^{ \pm}(a+c b) \cup-O^{ \pm}(c-a & a+b-c) \cup-O^{ \pm}(c-a a+b) \\
\cup-O^{ \pm}(a+2 b-c c-b) & (b+c>a+b>c-b),
\end{aligned} \\
O^{ \pm}(a b) \otimes O(c 0)=O^{ \pm}(a+c b) \cup-O^{ \pm}(c-a a+b-c) \cup-O^{ \pm}(c-a a+b) \\
\cup O^{ \pm}(c-a-2 b a+b) \quad(a+b<c-b) .
\end{gathered}
$$

The corresponding decompositions of products of antisymmetric and symmetric orbit functions can also be easily written down.

\section{Decomposition of antisymmetric $W$-orbit functions into antisymmetric $W^{\prime}$-orbit functions}

As in Section 7, for these decompositions it is enough to obtain the corresponding decompositions for signed orbits. For this reason, we shall deal mainly with signed orbits. Our reasoning here is very similar to that of Section 4 in [1].

\subsection{Introduction}

Let $R$ be a root system with a Weyl group $W$, and let $R^{\prime}$ be another root system which is a subset of the set $R$. Then a Weyl group $W^{\prime}$ for $R^{\prime}$ can be considered as a subgroup of $W$.

Let $O_{W}^{ \pm}(\lambda)$ be a signed $W$-orbit. The set of points of the usual orbit $O_{W}(\lambda)$ is invariant with respect to $W^{\prime}$. This means that the signed orbit $O_{W}^{ \pm}(\lambda)$ consists of signed $W^{\prime}$-orbits. In this section we deal with representing $O_{W}^{ \pm}(\lambda)$ as a union of signed $W^{\prime}$-orbits. Properties of such a representation depend on root systems $R$ and $R^{\prime}$ (or on Weyl groups $W$ and $W^{\prime}$ ). We distinguish two cases:

Case 1: Root systems $R$ and $R^{\prime}$ span vector spaces of the same dimension. In this case Weyl chambers for $W$ are smaller than Weyl chambers for $W^{\prime}$. Moreover, each Weyl chamber for $W^{\prime}$ consists of $\left|W / W^{\prime}\right|$ chambers for $W$. Let $D_{+}$be a dominant Weyl chamber for the root system $R$. Then a dominant Weyl chamber for $W^{\prime}$ consists of $W$-chambers $w_{i} D_{+}, i=1,2, \ldots, k, k=$ $\left|W / W^{\prime}\right|$, where $w_{i}, i=1,2, \ldots, k$, are representatives of cosets in $W / W^{\prime}$. If $\lambda$ does not lie on any wall of the dominant Weyl chamber $D_{+}$, then

$$
O_{W}^{ \pm}(\lambda)=\bigcup_{i=1}^{k}\left(\operatorname{det} w_{i}\right) O_{W^{\prime}}^{ \pm}\left(w_{i} \lambda\right)
$$

where $O_{W^{\prime}}^{ \pm}$are signed $W^{\prime}$-orbits. (Note that if $\operatorname{det} w_{i}=-1$, then $\left(\operatorname{det} w_{i}\right) O_{W^{\prime}}^{ \pm}\left(w_{i} \lambda\right)$ means the signed orbit $O_{W^{\prime}}^{ \pm}\left(w_{i} \lambda\right)$ in which each point is taken with opposite sign.)

Representing $\lambda$ by coordinates in $\omega$-basis it is necessary to take into account that coordinates of the same point in $\omega$-bases related to the root systems $R$ and $R^{\prime}$ are different. There exist matrices connecting coordinates in these different $\omega$-bases (see [32]). 
For expanding an antisymmetric $W$-orbit function into antisymmetric $W^{\prime}$-orbit functions it is necessary to take into account the formula (8.1). Namely, the following expansion

$$
\varphi_{\lambda}^{W}(x)=\sum_{i=1}^{k}\left(\operatorname{det} w_{i}\right) \varphi_{w_{i} \lambda}^{W^{\prime}}(x)
$$

corresponds to the decomposition (8.1).

Case 2: Root systems $R$ and $R^{\prime}$ span vector spaces of different dimensions. This case is more complicated. In order to represent $O_{W}^{ \pm}(\lambda)$ as a union of signed $W^{\prime}$-orbits, it is necessary to project points $\mu$ of $O_{W}^{ \pm}(\lambda)$ to the vector subspace $E_{n^{\prime}}$ spanned by $R^{\prime}$ and to select in the set of these projected points dominant points with respect to the root system $R^{\prime}$. Note that under projection, different points of $O_{W}^{ \pm}(\lambda)$ can give the same point in $E_{n^{\prime}}$. This leads to appearing of coinciding signed $W^{\prime}$-orbits in a representation of $O_{W}^{ \pm}(\lambda)$ as a union of $W^{\prime}$-orbits. Moreover, for some signed $W^{\prime}$-orbits their points must be taken with opposite signs.

As in the previous case, under expansion of an antisymmetric $W$-orbit function $\varphi_{\lambda}(x)$ into antisymmetric $W^{\prime}$-orbit functions we have to consider $\varphi_{\lambda}(x)$ on the subspace $E_{n^{\prime}} \subset E_{n}$ and to take into account the corresponding decomposition of the signed orbit $O_{W}^{ \pm}(\lambda)$. For this reason, below in this section we consider decomposition of signed $W$-orbits into $W^{\prime}$-orbits. They uniquely determine the corresponding expansions for antisymmetric orbit functions.

\subsection{Decomposition of signed $W_{A_{n}}$-orbits into $W_{A_{n-1}}$-orbits}

For such decomposition it is convenient to represent orbit elements in orthogonal coordinates (see Section 3). Let $O^{ \pm}(\lambda) \equiv O^{ \pm}\left(m_{1}, m_{2}, \ldots, m_{n+1}\right)$ be a signed $W_{A_{n}}$-orbit with dominant element $\lambda=\left(m_{1}, m_{2}, \ldots, m_{n+1}\right)$, where

$$
m_{1}>m_{2}>\cdots>m_{n}>m_{n+1} .
$$

The orthogonal coordinates $m_{1}, m_{2}, \ldots, m_{n+1}$ satisfy the conditions $m_{1}+m_{2}+\cdots+m_{n+1}=0$. However, we may add to all coordinates $m_{i}$ the same real number, since under this procedure the $\omega$-coordinates $\lambda_{i}=m_{i}-m_{i+1}, i=1,2, \ldots, n$ do not change (see Section 3 ).

The signed orbit $O^{ \pm}(\lambda)$ consists of all points

$$
w\left(m_{1}, m_{2}, \ldots, m_{n+1}\right)=\left(m_{i_{1}}, m_{i_{2}}, \ldots, m_{i_{n+1}}\right), \quad w \in W_{A_{n}},
$$

where $\left(i_{1}, i_{2}, \ldots, i_{n+1}\right)$ is a permutation of the numbers $1,2, \ldots, n+1$, determined by $w$. The sign of $(\operatorname{det} w)$ is attached to such point. Points of $O^{ \pm}(\lambda)$ belong to the vector space $E_{n+1}$. We restrict these points to the vector subspace $E_{n}$, spanned by the simple roots $\alpha_{1}, \alpha_{2}, \ldots, \alpha_{n-1}$ of $A_{n}$, which form a set of simple roots of $A_{n-1}$. This restriction is reduced to removing the last coordinate $m_{i_{n+1}}$ in points $\left(m_{i_{1}}, m_{i_{2}}, \ldots, m_{i_{n+1}}\right)$ of the signed orbit $O^{ \pm}(\lambda)$ (see $\left.(8.2)\right)$. As a result, we obtain a set of points

$$
\left(m_{i_{1}}, m_{i_{2}}, \ldots, m_{i_{n}}\right)
$$

received from the points (8.2). The point (8.3) is dominant if and only if

$$
m_{i_{1}} \geq m_{i_{2}} \geq \cdots \geq m_{i_{n}}
$$

It is easy to see that after restriction to $E_{n}$ (that is, under removing the last coordinate) we obtain from the set of points (8.2) the following set of dominant elements:

$$
\left(m_{1}, \ldots, m_{i-1}, \hat{m}_{i}, m_{i+1}, \ldots, m_{n+1}\right), \quad i=1,2, \ldots, n+1,
$$

where a hat over $m_{i}$ means that the coordinate $m_{i}$ must be omitted. 
Thus, the signed $W_{A_{n}}$-orbit $O^{ \pm}\left(m_{1}, m_{2}, \ldots, m_{n+1}\right)$ consists of the following signed $W_{A_{n-1} \text { - }}$ orbits:

$$
O^{ \pm}\left(m_{1}, \ldots, m_{i-1}, \hat{m}_{i}, m_{i+1}, \ldots, m_{n+1}\right), \quad i=1,2, \ldots, n+1 .
$$

Each of these signed orbits must be taken with a coefficient +1 or -1 . Moreover, a coefficient at the orbit $O^{ \pm}\left(m_{1}, \ldots, m_{i-1}, \hat{m}_{i}, m_{i+1}, \ldots, m_{n+1}\right)$ is 1 , if after $\hat{m}_{i}$ in the point

$$
\left(m_{1}, \ldots, m_{i-1}, \hat{m}_{i}, m_{i+1}, \ldots, m_{n+1}\right)
$$

a number of coordinates is even, and -1 otherwise. This statement completely determines an expansion of the antisymmetric orbit function $\varphi_{\left(m_{1}, m_{2}, \ldots, m_{n+1}\right)}^{\left(W_{A_{n}}\right)}$ into antisymmetric $W_{A_{n-1}}$-orbit function:

$$
\varphi_{\left(m_{1}, m_{2}, \ldots, m_{n+1}\right)}(x)=\sum_{i=1}^{n+1}\left(\operatorname{det} w\left(m_{i}\right)\right) \varphi_{\left(m_{1}, \ldots, m_{i-1}, \hat{m}_{i}, m_{i+1}, \ldots, m_{n+1}\right)}(x),
$$

where $w\left(m_{i}\right)$ is the permutation which sends the coordinate $m_{i}$ to the end, not changing an order of other coordinates.

\subsection{Decomposition of signed $W_{A_{n-1}}$-orbits into $W_{A_{p-1}} \times W_{A_{q-1}}$-orbits, $p+q=n$}

Again we use orthogonal coordinates for orbit elements. We take in the system of simple roots $\alpha_{1}, \alpha_{2}, \ldots, \alpha_{n-1}$ of $A_{n-1}$ two parts as $\alpha_{1}, \alpha_{2}, \ldots, \alpha_{p-1}$ and $\alpha_{p+1}, \alpha_{p+2}, \ldots, \alpha_{p+q-1} \equiv \alpha_{n-1}$. The first part determines $W_{A_{p-1}}$ and the second part generates $W_{A_{q-1}}$. We consider a signed $W_{A_{n-1}{ }^{-}}$ orbit $O^{ \pm}(\lambda)$, where $\lambda=\left(m_{1}, m_{2}, \ldots, m_{n}\right), m_{1}>m_{2}>\cdots>m_{n}$. This orbit consists of points

$$
w\left(m_{1}, m_{2}, \ldots, m_{n}\right)=\left(m_{i_{1}}, m_{i_{2}}, \ldots, m_{i_{n}}\right), \quad w \in W_{A_{n-1}},
$$

where $\left(i_{1}, i_{2}, \ldots, i_{n}\right)$ is a permutation of the numbers $1,2, \ldots, n$. We restrict points (8.4) to the vector subspace $E_{p} \times E_{q}$ spanned by the simple roots $\alpha_{1}, \alpha_{2}, \ldots, \alpha_{p-1}$ and $\alpha_{p+1}, \alpha_{p+2}, \ldots, \alpha_{n-1}$, respectively. Under restriction the point (8.4) turns into the point

$$
\left(m_{i_{1}}, m_{i_{2}}, \ldots, m_{i_{p}}\right)\left(m_{i_{p+1}}, m_{i_{p+1}}, \ldots, m_{i_{n}}\right) .
$$

In order to determine a set of signed $W_{A_{p-1}} \times W_{A_{q-1}}$-orbits contained in the signed orbit $O^{ \pm}(\lambda)$ we have to choose from (8.4) all elements for which

$$
m_{i_{1}}>m_{i_{2}}>\cdots>m_{i_{p}}, \quad m_{i_{p+1}}>m_{i_{p+2}}>\cdots>m_{i_{n}} .
$$

To find this set of points we have to take all subsets $m_{i_{1}}, m_{i_{2}}, \ldots, m_{i_{p}}$ in the set $m_{1}, m_{2}, \ldots, m_{n}$ such that $m_{i_{1}}>m_{i_{2}}>\cdots>m_{i_{p}}$. Let $\Sigma$ denote the collection of such subsets. Then $O^{ \pm}(\lambda)$ consists of signed $W_{A_{p-1}} \times W_{A_{q-1}}$-orbits

$$
O^{ \pm}\left(\left(m_{i_{1}}, m_{i_{2}}, \ldots, m_{i_{p}}\right)\left(m_{j_{1}}, m_{j_{2}}, \ldots, m_{j_{q}}\right)\right), \quad\left(m_{i_{1}}, m_{i_{2}}, \ldots, m_{i_{p}}\right) \in \Sigma,
$$

where $\left(m_{j_{1}}, m_{j_{2}}, \ldots, m_{j_{q}}\right)$ is a supplement of the subset $\left(m_{i_{1}}, m_{i_{2}}, \ldots, m_{i_{p}}\right)$ in the whole set $\left(m_{1}, m_{2}, \ldots, m_{n}\right)$, taken in such an order that $m_{j_{1}}>m_{j_{2}}>\cdots>m_{j_{q}}$. Each of these $W_{A_{p-1}} \times$ $W_{A_{q-1}}$-orbits is contained in $O^{ \pm}(\lambda)$ only once. Each such a signed orbit is contained in the signed orbit $O^{ \pm}(\lambda)$ with sign "+" if the set of numbers

$$
\left(m_{i_{1}}, m_{i_{2}}, \ldots, m_{i_{p}}, m_{j_{1}}, m_{j_{2}}, \ldots, m_{j_{q}}\right)
$$

from $(8.5)$ is obtained from the set $(1,2, \ldots, n)$ by an even permutation and with sign "-"

otherwise. The corresponding expansions of antisymmetric orbit functions $\varphi_{\lambda}^{\left(W_{A_{n-1}}\right)}(x)$ into antisymmetric $W_{A_{p-1}} \times W_{A_{q-1}}$-orbit functions is evident. 


\subsection{Decomposition of signed $W_{B_{n}}$-orbits into $W_{B_{n-1}}$-orbits and of signed $W_{C_{n}}$-orbits into $W_{C_{n-1}}$-orbits}

Decomposition of signed $W_{B_{n}}$-orbits and decomposition of signed $W_{C_{n}}$-orbits are fulfilled in the same way. For this reason, we give a proof only for the case of signed $W_{C_{n}}$-orbits.

A set of simple roots of $C_{n}$ consists of roots $\alpha_{1}, \alpha_{2}, \ldots, \alpha_{n}$. The roots $\alpha_{2}, \ldots, \alpha_{n}$ constitute a set of simple roots of $C_{n-1}$. They span the subspace $E_{n-1}$.

For determining elements $\lambda$ of $E_{n}$ we use orthogonal coordinates $m_{1}, m_{2}, \ldots, m_{n}$. Then $\lambda$ is strictly dominant if and only if

$$
m_{1}>m_{2}>\cdots>m_{n}>0 \text {. }
$$

Then the signed orbit $O^{ \pm}(\lambda)$ consists of all points

$$
w\left(m_{1}, m_{2}, \ldots, m_{n}\right)=\left( \pm m_{i_{1}}, \pm m_{i_{2}}, \ldots, \pm m_{i_{n}}\right), \quad w \in W_{C_{n}}
$$

where $\left(i_{1}, i_{2}, \ldots, i_{n}\right)$ is a permutation of the set $1,2, \ldots, n$, and all combinations of signs are possible.

Restriction of elements (8.6) to the vector subspace $E_{n-1}$, defined above, reduces to removing the first coordinate $\pm m_{i_{1}}$ in (8.6). As a result, we obtain from the set of points (8.6) the collection

$$
\left( \pm m_{i_{2}}, \pm m_{i_{3}}, \ldots, \pm m_{i_{n}}\right), \quad w \in W_{C_{n}} .
$$

Only the points $\left(m_{i_{2}}, m_{i_{3}}, \ldots, m_{i_{n-1}}, m_{i_{n}}\right)$ with positive coordinates may be dominant. Moreover, such a point is dominant if and only if

$$
m_{i_{2}}>m_{i_{3}}>\cdots>m_{i_{n}} .
$$

Therefore, under restriction of points (8.6) to $E_{n-1}$ we obtain the following strictly $W_{C_{n-1}}{ }^{-}$ dominant elements:

$$
\left(m_{1}, m_{2}, \ldots, m_{i-1}, \hat{m}_{i}, m_{i+1}, \ldots, m_{n}\right), \quad i=1,2, \ldots, n
$$

where a hat over $m_{i}$ means that the coordinate $m_{i}$ must be omitted. Moreover, the element (8.7) with fixed $i$ can be obtained from two elements in (8.6), namely, from $\left(m_{1}, m_{2}, \ldots, m_{i-1}, \pm m_{i}\right.$, $\left.m_{i+1}, \ldots, m_{n}\right)$. In the signed orbit $O^{ \pm}\left(m_{1}, m_{2}, \ldots, m_{n}\right)$ these two elements have opposite signs.

Thus, the signed $W_{C_{n}}$-orbits $O^{ \pm}\left(m_{1}, m_{2}, \ldots, m_{n}\right)$ consists of the following signed $W_{C_{n-1}}$ orbits:

$$
O^{ \pm}\left(m_{1}, m_{2}, \ldots, m_{i-1}, \hat{m}_{i}, m_{i+1}, \ldots, m_{n}\right), \quad i=1,2, \ldots, n .
$$

Each such signed $W_{C_{n-1}}$-orbit is contained in $O^{ \pm}\left(m_{1}, m_{2}, \ldots, m_{n}\right)$ twice with opposite signs.

Therefore, a restriction of the antisymmetric orbit function $\varphi_{\left(m_{1}, m_{2}, \ldots, m_{n}\right)}$ to the subspace $E_{n-1}$, described above, vanishes.

For $W_{B_{n}}$-orbits we have similar assertions. A signed $W_{B_{n}}$-orbits $O^{ \pm}\left(m_{1}, m_{2}, \ldots, m_{n}\right), m_{1}>$ $m_{2}>\cdots>m_{n}>0$, consists of $W_{B_{n-1}}$-orbits

$$
O^{ \pm}\left(m_{1}, m_{2}, \ldots, m_{i-1}, \hat{m}_{i}, m_{i+1}, \ldots, m_{n}\right), \quad i=1,2, \ldots, n,
$$

and each such orbit is contained in the decomposition two times (with opposite signs), that is, a restriction of the antisymmetric orbit function $\varphi_{\left(m_{1}, m_{2}, \ldots, m_{n}\right)}$ of $B_{n}$ to the subspace $E_{n-1}$, described above, vanishes. 


\subsection{Decomposition of signed $W_{C_{n}}$-orbits into $W_{A_{p-1}} \times W_{C_{q}}$-orbits, $p+q=n$}

If $\alpha_{1}, \alpha_{2}, \ldots, \alpha_{n}$ are simple roots for $C_{n}$, then $\alpha_{1}, \alpha_{2}, \ldots, \alpha_{p-1}$ are simple roots for $A_{p-1}$ (they can be embedded into the linear subspace $\left.E_{p}\right)$ and $\alpha_{p+1}, \alpha_{p+2}, \ldots, \alpha_{n}$ are simple roots for $C_{q}$ (they generate the linear subspace $E_{q}$ ).

We use orthogonal coordinates for elements of $E_{n}$ and consider a signed $W_{C_{n}}$-orbit $O^{ \pm}(\lambda)$, $m_{1}>m_{2}>\cdots>m_{n}>0$. This orbit consists of all points (8.6). Restriction of these points to the vector subspace $E_{p} \times E_{q}$ reduces to splitting of coordinates (8.6) into two parts:

$$
\left( \pm m_{i_{1}}, \pm m_{i_{2}}, \ldots, \pm m_{i_{p}}\right)\left( \pm m_{i_{p+1}}, \ldots, \pm m_{i_{n}}\right) .
$$

Due to the condition $m_{1}>m_{2}>\cdots>m_{n}>0$, these elements do not lie on walls of the $W_{A_{p-1}} \times W_{C_{q}}$-chambers. We have to choose dominant elements (with respect to the Weyl group $W_{A_{p-1}} \times W_{C_{q}}$ ) in the set of points (8.8). The conditions of dominantness for elements of $E_{p}$ and $E_{q}$ show that only the elements

$$
\left(m_{i_{1}}, \ldots, m_{i_{j}},-m_{i_{j+1}} \cdots,-m_{i_{p}}\right)\left(m_{i_{p+1}}, \ldots, m_{i_{n}}\right), \quad j=0,1,2, \ldots, p,
$$

satisfying the conditions

$$
m_{i_{1}}>m_{i_{2}}>\cdots>m_{i_{j}}, \quad m_{i_{j+1}}<m_{i_{j+2}}<\cdots<m_{i_{p}}, \quad m_{i_{p+1}}>m_{i_{p+2}}>\cdots>m_{i_{n}},
$$

are dominant. Moreover, each such point is contained in the signed $W_{C_{n}}$-orbit $O^{ \pm}(\lambda)$ only once. This assertion completely determines a list of signed $W_{A_{p-1}} \times W_{C_{q}}$-orbits in $O^{ \pm}(\lambda)$. Each signed $W_{A_{p-1}} \times W_{C_{q}}$-orbit is contained in $O^{ \pm}(\lambda)$ only once.

This assertion uniquely determines a list of antisymmetric $W_{A_{p-1}} \times W_{C_{q}}$-orbit functions, contained in the antisymmetric $W_{C_{n}}$-orbit function $\varphi_{\lambda}$. However, it is necessary to determine signs of antisymmetric $W_{A_{p-1}} \times W_{C_{q}}$-orbit functions in the decomposition. It is easily made by using the description of the Weyl groups in the Euclidean space $E_{n}$ with orthogonal coordinates.

\subsection{Decomposition of signed $W_{D_{n}}$-orbits into signed $W_{D_{n-1}}$-orbits}

Assume that $\alpha_{1}, \alpha_{2}, \ldots, \alpha_{n}$ is the set of simple roots of $D_{n}, n>4$. Then $\alpha_{2}, \ldots, \alpha_{n}$ are simple roots of $D_{n-1}$. The last roots span the subspace $E_{n-1}$.

For elements $\lambda$ of $E_{n}$ we again use orthogonal coordinates $m_{1}, m_{2}, \ldots, m_{n}$. Then $\lambda$ is strictly dominant if and only if $m_{1}>m_{2}>\cdots>m_{n-1}>\left|m_{n}\right|$. We assume that $\lambda$ satisfies the condition

$$
m_{1}>m_{2}>\cdots>m_{n}>0 .
$$

Then the signed orbit $O^{ \pm}(\lambda)$ consists of all points

$$
w\left(m_{1}, m_{2}, \ldots, m_{n}\right)=\left( \pm m_{i_{1}}, \pm m_{i_{2}}, \ldots, \pm m_{i_{n}}\right), \quad w \in W_{D_{n}},
$$

where $\left(i_{1}, i_{2}, \ldots, i_{n}\right)$ is a permutation of the numbers $1,2, \ldots, n$ and there exists an even number of signs "-". Restriction of elements (8.9) to the subspace $E_{n-1}$ reduces to removing the first coordinate $\pm m_{i_{1}}$ in (8.9). As a result, we obtain from the set of points (8.9) the collection

$$
\left( \pm m_{i_{2}}, \pm m_{i_{3}}, \ldots, \pm m_{i_{n}}\right), \quad w \in W_{D_{n}}
$$

where a number of signs "-" may be either even or odd. Only points of the form $\left(m_{i_{2}}, m_{i_{3}}, \ldots\right.$, $\left.m_{i_{n-1}}, \pm m_{i_{n}}\right)$ may be dominant. Moreover, such a point is dominant if and only if

$$
m_{i_{2}}>m_{i_{3}}>\cdots>m_{i_{n-1}}>\left|m_{i_{n}}\right| .
$$


Therefore, under restriction of points $(8.9)$ to $E_{n-1}$ we obtain the following $W_{D_{n-1}}$-dominant elements:

$$
\left(m_{1}, m_{2}, \ldots, m_{i-1}, \hat{m}_{i}, m_{i+1}, \ldots, m_{n-1}, \pm m_{n}\right), \quad i=1,2, \ldots, n,
$$

where a hat over $m_{i}$ means that the coordinate $m_{i}$ must be omitted. Moreover, the element (8.10) with fixed $i$ can be obtained only from one element in (8.9), namely, from element $\left(m_{1}, m_{2}, \ldots, m_{i-1}, \pm m_{i}, m_{i+1}, \ldots, \pm m_{n}\right)$, where at $m_{i}$ and $m_{n}$ signs are coinciding.

Thus, the signed $W_{D_{n}}$-orbit $O^{ \pm}\left(m_{1}, m_{2}, \ldots, m_{n}\right)$ with $m_{1}>m_{2}>\cdots>m_{n}>0$ consists of the following signed $W_{D_{n-1}}$-orbits:

$$
O^{ \pm}\left(m_{1}, m_{2}, \ldots, m_{i-1}, \hat{m}_{i}, m_{i+1}, \ldots, \pm m_{n}\right), \quad i=1,2, \ldots, n .
$$

Each such signed $W_{D_{n-1}}$-orbit is contained in $O^{ \pm}\left(m_{1}, m_{2}, \ldots, m_{n}\right)$ only once (with sign "+ " or "- "). A sign of such an orbit depends on a number $i$ and on a sign at $m_{n}$. This sign is uniquely determined by the sign ( $\operatorname{det} w$ ) of the corresponding element $w$ of $W_{D_{n}}$.

It is shown similarly that the signed $W_{D_{n}}$-orbit

$$
O^{ \pm}\left(m_{1}, \ldots, m_{n-1},-m_{n}\right), \quad m_{1}>m_{2}>\cdots>m_{n}>0
$$

consists of the same signed $W_{D_{n-1}}$-orbits as the $W_{D_{n}}$-orbits $O^{ \pm}\left(m_{1}, \ldots, m_{n-1}, m_{n}\right)$ with the same numbers $m_{1}, \ldots, m_{n-1}, m_{n}$ does.

The above assertions uniquely determine expansions for the corresponding antisymmetric $W_{D_{n}}$-orbit functions.

\subsection{Decomposition of signed $W_{D_{n}}$-orbits into $W_{A_{p-1}} \times W_{D_{q}}$-orbits, $p+q=n, q \geq 4$}

If $\alpha_{1}, \alpha_{2}, \ldots, \alpha_{n}$ are simple roots for $D_{n}$, then $\alpha_{1}, \alpha_{2}, \ldots, \alpha_{p-1}$ are simple roots for $A_{p-1}$ (they can be embedded into the Euclidean subspace $\left.E_{p}\right)$ and $\alpha_{p+1}, \alpha_{p+2}, \ldots, \alpha_{n}$ are simple roots for $D_{q}$ (they generate the Euclidean subspace $E_{q}$ ).

We use orthogonal coordinates in $E_{n}$ and consider signed $W_{D_{n}}$-orbits $O^{ \pm}(\lambda)$ with $\lambda=$ $\left(m_{1}, m_{2}, \ldots, m_{n}\right)$ such that $m_{1}>m_{2}>\cdots>m_{n}>0$. The orbit $O(\lambda)$ consists of all points (8.9). Restriction of these points to the vector subspace $E_{p-1} \times E_{q}$ reduces to splitting the set of coordinates (8.9) into two parts:

$$
\left( \pm m_{i_{1}}, \pm m_{i_{2}}, \ldots, \pm m_{i_{p}}\right)\left( \pm m_{i_{p+1}}, \ldots, \pm m_{i_{n}}\right) .
$$

Due to the condition $m_{1}>m_{2}>\cdots>m_{n}>0$, these elements do not lie on walls of the $W_{A_{p-1}} \times W_{D_{q}}$-chambers. We have to choose dominant elements (with respect to the Weyl group $W_{A_{p-1}} \times W_{D_{q}}$ ) in the set of points (8.11). The conditions of dominantness for elements of $E_{p}$ and $E_{q}$ show that only the elements

$$
\left(m_{i_{1}}, \ldots, m_{i_{j}},-m_{i_{j+1}} \ldots,-m_{i_{p}}\right)\left(m_{i_{p+1}}, \ldots, \pm m_{i_{n}}\right), \quad j=0,1,2, \ldots, p,
$$

having even number of sign minus and satisfying the conditions

$$
m_{i_{1}}>m_{i_{2}}>\cdots>m_{i_{j}}, \quad m_{i_{j+1}}<m_{i_{j+2}}<\cdots<m_{i_{p}}, \quad m_{i_{p+1}}>m_{i_{p+2}}>\cdots>m_{i_{n}},
$$

are dominant. Moreover, each such point is contained in the $W_{D_{n}}$-orbit $O^{ \pm}(\lambda)$ only once. These assertions completely determine a list of signed $W_{A_{p-1}} \times W_{D_{q}}$-orbits in the signed $W_{D_{n}}$-orbit $O^{ \pm}(\lambda)$. All signed $W_{A_{p-1}} \times W_{D_{q}}$-orbits are contained in $O^{ \pm}(\lambda)$ with multiplicity 1 and with sign "+" or "-". This determines uniquely expansions for the corresponding antisymmetric $W_{D_{n}}$-orbit functions. 


\section{Characters of representations and antisymmetric orbit functions}

Antisymmetric orbit functions $\varphi_{\lambda}(x)$ with $\lambda \in P_{+}^{+}$are closely related to characters of irreducible representations of the corresponding compact Lie group $G$. This relation serves for derivations of some properties of antisymmetric orbit functions.

\subsection{Connection of characters with orbit functions}

To each Coxeter-Dynkin diagram there corresponds a connected compact semisimple Lie group $G$. Let us fix a Coxeter-Dynkin diagram and, therefore, a connected compact Lie group $G$. A complex valued function $f(g)$ on $G$ satisfying the condition

$$
f(g)=f\left(h g h^{-1}\right), \quad h \in G,
$$

is called a class function. It is constant on classes of conjugate elements.

For simplicity, we assume that $G$ is realized by matrices such that the set of its diagonal matrices constitute a Cartan subgroup, which will be denoted by $H$. This subgroup can be identified with the $n$-dimensional torus $\mathrm{T}$, where $n$ is a rank of the group $G$. The subgroup $H$ can be represented as $H=\exp (\mathfrak{i h})$, where $\mathfrak{h}$ is a real form of an appropriate Cartan subalgebra of the complex semisimple Lie algebra, determined by the Coxeter-Dynkin diagram.

It is well-known that each element $g$ of $G$ is conjugate to some element of $H$, that is, class functions are uniquely determined by their values on $H$.

There exists a one-to-one correspondence between irreducible unitary representations of the group $G$ and integral highest weights $\lambda \in P_{+}$, where $P_{+}$is determined by the Coxeter-Dynkin diagram. The irreducible representation, corresponding to a highest weight $\lambda$, will be denoted by $T_{\lambda}$. The representation $T_{\lambda}$ and its properties are determined by its character $\chi_{\lambda}(g)$, which is defined as the trace of $T_{\lambda}(g)$ :

$$
\chi_{\lambda}(g)=\operatorname{Tr} T_{\lambda}(g), \quad g \in G .
$$

Since $\operatorname{Tr} T_{\lambda}\left(h g h^{-1}\right)=\operatorname{Tr} T_{\lambda}(g), h \in G$, the character $\chi_{\lambda}$ is a class function, that is, it is uniquely determined by its values on the subgroup $H$.

All the operators $T_{\lambda}(h), h \in H$, are diagonal with respect to an appropriate basis of the representation space (this basis is called a weight basis) and their diagonal matrix elements are of the form $e^{2 \pi \mathrm{i}\langle\mu, x\rangle}$, where $\mu \in P$ is a weight of the representation $T_{\lambda}, x=\left(x_{1}, x_{2}, \ldots, x_{n}\right)$ are coordinates of an element $t$ of the Cartan subalgebra $\mathfrak{h}$ in an appropriate coordinate system (that is, coordinates on the torus $\mathrm{T}$ ) and $\langle\cdot, \cdot\rangle$ is an appropriate bilinear form, which can be chosen coinciding with the scalar product on $E_{n}$, considered above. Then the character $\chi_{\lambda}(h)$ is a linear combination of the diagonal matrix elements:

$$
\chi_{\lambda}(h)=\sum_{\mu \in D(\lambda)} c_{\lambda}^{\mu} e^{2 \pi \mathrm{i}\langle\mu, x\rangle},
$$

where $D(\lambda)$ is the set of all weights of the irreducible representation $T_{\lambda}$ and $c_{\lambda}^{\mu}$ is a multiplicity of the weight $\mu \in D(\lambda)$ in the representation $T_{\lambda}$. It is known from representation theory that the weight system $D(\lambda)$ of $T_{\lambda}$ is invariant with respect to the Weyl group $W$, corresponding to the Coxeter-Dynkin diagram, and $c_{\lambda}^{w \mu}=c_{\lambda}^{\mu}, w \in W$, for each $\mu \in D(\lambda)$. This means that the character $\chi_{\lambda}(h)$ can be represented as

$$
\chi_{\lambda}(h)=\sum_{\mu \in D_{+}(\lambda)} c_{\lambda}^{\mu} \phi_{\mu}(x),
$$


where $D_{+}(\lambda)$ is the set of all dominant weights in $D(\lambda)$ and $\phi_{\mu}(x)$ is a symmetric orbit function, corresponding to the weight $\mu \in D_{+}(\lambda)$. Representing $\chi_{\lambda}(h)$ as $\chi_{\lambda}(x)$, where $x=\left(x_{1}, x_{2}, \ldots, x_{n}\right)$ are coordinates corresponding to the element $t \in \mathfrak{h}$ such that $h=\exp 2 \pi \mathrm{i} t$, we can make an analytic continuation of both sides of (9.2) to the $n$-dimensional Euclidean space $E_{n}$. Since the right hand side of (9.2) is invariant under transformations from the affine Weyl group $W^{\text {aff }}$, corresponding to the Weyl group $W$, the function $\chi_{\lambda}(x)$ is also invariant under the affine Weyl group $W^{\text {aff }}$. That is, it is enough to define $\chi_{\lambda}(x)$ only on the fundamental domain $F$ of the group $W^{\text {aff }}$. To this fundamental domain $F$ there corresponds a fundamental domain (we denote it by $\tilde{F}$ ) in the subgroup $H$ (and on the torus $\mathrm{T}$ ).

Many properties of orbit functions follow from properties of characters $\chi_{\lambda}$, and we consider them as known from representation theory.

The well-known Weyl formula for characters of irreducible representations of the group $G$ states [33] that under appropriate selection of coordinates $\left(x_{1}, x_{1}, \ldots, x_{n}\right)$ of points $h \in H$ we have

$$
\chi_{\lambda}(h)=\frac{\sum_{w \in W}(\operatorname{det} w) e^{2 \pi \mathrm{i}\langle\lambda+\rho, x\rangle}}{\sum_{w \in W}(\operatorname{det} w) e^{2 \pi \mathrm{i}\langle\rho, x\rangle}}=\frac{\varphi_{\lambda+\rho}(x)}{\varphi_{\rho}(x)},
$$

where, as before, $\rho=\frac{1}{2} \sum_{\alpha>0} \alpha$. This is why antisymmetric orbit functions are so important for representation theory. Note that for dominant $\lambda \in P_{+}$the element $\lambda+\rho$ is strictly dominant; for this reason, the antisymmetric orbit function $\varphi_{\lambda+\rho}(x)$ in (9.3) does not vanish for $\lambda \in P_{+}$.

\subsection{Orthogonality of characters}

The relation (9.3) between characters of irreducible representations of compact Lie groups and the corresponding antisymmetric orbit functions leads to a simple proof of orthogonality of irreducible characters $\chi_{\lambda}(h) \equiv \chi_{\lambda}(x)$. Indeed, due to the orthogonality (5.2) for antisymmetric orbit functions and to the relation (9.3) we have

$$
\int_{F} \varphi_{\lambda+\rho}(x) \overline{\varphi_{\lambda^{\prime}+\rho}(x)} d x=\int_{F} \chi_{\lambda}(x) \chi_{\lambda^{\prime}}(x)\left|\varphi_{\rho}(x)\right|^{2} d x=\delta_{\lambda \lambda^{\prime}}
$$

that is, irreducible characters are orthogonal on $F$ with respect to the measure $\left|\varphi_{\rho}(x)\right|^{2} d x$. The expressions for the function $\varphi_{\rho}(x)$ for the classical compact Lie groups are given in Subsection 5.9.

\subsection{Relations for antisymmetric orbit functions}

The formulas (9.2) and (9.3) are a source of relations for antisymmetric orbit functions.

Comparing the expressions (9.2) and (9.3) for characters, one gets the relation

$$
\varphi_{\lambda+\rho}(x)=\sum_{\mu \in D_{+}(\lambda)} c_{\lambda}^{\mu} \phi_{\mu}(x) \varphi_{\rho}(x)
$$

where, as before, $c_{\lambda}^{\mu}$ are multiplicities of weights $\mu$ in the irreducible representation $T_{\lambda}$ of $G$.

Let $T_{\lambda}$ and $T_{\mu}$ be two irreducible representations of $G$. Then their tensor product decomposes as a direct sum of irreducible representations of $G$ as

$$
T_{\lambda} \otimes T_{\mu}=\sum_{\nu \in P_{+}} m_{\nu}^{\lambda \mu} T_{\nu}
$$


where $m_{\nu}^{\lambda \mu}$ is a multiplicity of the irreducible representation $T_{\nu}$ in the tensor product. Since $T_{\lambda}$ and $T_{\mu}$ are finite dimensional representations, the sum on the right hand side of (9.5) is finite. To the decomposition (9.5) there corresponds a relation for characters,

$$
\chi_{\lambda}(x) \chi_{\mu}(x)=\sum_{\nu \in P_{+}} m_{\nu}^{\lambda \mu} \chi_{\nu}(x) .
$$

Due to (9.3) it can be written as

$$
\frac{\varphi_{\lambda+\rho}(x)}{\varphi_{\rho}(x)} \frac{\varphi_{\mu+\rho}(x)}{\varphi_{\rho}(x)}=\sum_{\nu \in P_{+}} m_{\mu \nu}^{\lambda} \frac{\varphi_{\nu+\rho}(x)}{\varphi_{\rho}(x)}
$$

where summation is such as in (9.6). Therefore, we have the following expression for a product of two antisymmetric orbit functions:

$$
\varphi_{\lambda+\rho}(x) \varphi_{\mu+\rho}(x)=\sum_{\nu \in P_{+}} m_{\nu}^{\lambda \mu} \varphi_{\nu+\rho}(x) \varphi_{\rho}(x) .
$$

In particular, if $\mu=\omega_{i}$, where $\omega_{i}$ is $i$-th fundamental wight of the group $G$, then this formula takes the form

$$
\varphi_{\lambda+\rho}(x) \varphi_{\omega_{i}+\rho}(x)=\sum_{\nu \in P_{+}} m_{\nu}^{\lambda \omega_{i}} \varphi_{\nu+\rho}(x) \varphi_{\rho}(x) .
$$

Since multiplicities of irreducible constituents in the tensor product $T_{\lambda} \otimes T_{\omega_{i}}$ for many groups can be found in a simple way (in many cases these multiplicities are equal to 1; for example, if $i=1$, then for the groups $S U(n)$ and $S O(n)$ they do not exceed 1), then this formula can be considered as a recurrence relation for antisymmetric orbit functions.

\section{Antisymmetric orbit function transforms}

As in the case of symmetric orbit functions, antisymmetric orbit functions determine certain orbit function transforms which generalize the sine transform (in the case of symmetric orbit functions these transforms generalize the cosine transform) [29, 31].

As in the case of symmetric orbit functions, antisymmetric orbit functions determine three types of orbit function transforms: the first one is related to the antisymmetric orbit functions $\varphi_{\lambda}(x)$ with integral $\lambda$, the second one is related to $\varphi_{\lambda}(x)$ with dominant $\lambda \in E_{n}$, and the third one is the related discrete transforms.

\subsection{Decomposition in antisymmetric orbit functions on $F$}

Let $f(g)$ be a continuous class function on $G$ (see Subsection 9.1). It defines a continuous function on the commutative subgroup $H$. We assume that this function on $H$ has continuous partial derivatives of all orders with respect to analytic parameters on $H$. Such function $f$ can be decomposed in characters of irreducible unitary representations of $G$ :

$$
f(h)=\sum_{\lambda \in P_{+}} c_{\lambda} \chi_{\lambda}(h)
$$

We see from this decomposition that each class function is symmetric with respect to the corresponding affine Weyl group $W^{\text {aff }}$ (since characters $\chi_{\lambda}$ admit this symmetry) and, therefore, is uniquely determined by its values on the fundamental domain $\tilde{F}$ in $H$. 
Going to the coordinate description of the points $h \in H$ (see Subsection 9.1), we obtain

$$
f(x)=\sum_{\lambda \in P_{+}} c_{\lambda} \chi_{\lambda}(x), \quad x \in E_{n} .
$$

Taking into account formula (9.3) for the characters we receive

$$
\varphi_{\rho}(x) f(x)=\sum_{\lambda \in P_{+}} c_{\lambda} \varphi_{\lambda+\rho}(x) .
$$

Due to Proposition 7, we may state that any antisymmetric (with respect to the affine Weyl group $W^{\text {aff }}$ ) continuous function $f$ on $E_{n}$, which has continuous derivatives and vanishes on the boundary $\partial F$ of the fundamental domain $F$, can be represented in the form $f(x)=\varphi_{\rho}(x) \tilde{f}(x)$, where $\tilde{f}(x)$ is a symmetric (with respect to $W^{\text {aff }}$ ) continuous function on $E_{n}$ with continuous derivatives. Thus, due to (10.2) we may state that any antisymmetric (with respect to $W^{\text {aff }}$ ) continuous function $f$ on $E_{n}$, which has continuous derivatives and vanishes on the boundary $\partial F$, can be decomposed in antisymmetric orbit functions $\varphi_{\lambda}, \lambda \in P_{+}^{+}$,

$$
f(x)=\sum_{\lambda \in P_{+}^{+}} c_{\lambda} \varphi_{\lambda}(x) .
$$

By the orthogonality relation (5.2) for antisymmetric orbit functions, the coefficients $c_{\lambda}$ in this decomposition are determined by the formula

$$
c_{\lambda}=\int_{F} f(x) \overline{\varphi_{\lambda}(x)} d x
$$

Moreover, the Plancherel formula

$$
\sum_{\lambda \in P_{+}}\left|c_{\lambda}\right|^{2}=\int_{F}|f(x)|^{2} d x
$$

holds, which means that the Hilbert spaces with the appropriate scalar products are isometric.

Formula (10.4) is the antisymmetrized Fourier transform of the function $f(x)$. Formula (10.3) gives an inverse transform. Formulas (10.3) and (10.4) give the orbit function transforms corresponding to antisymmetric orbit functions $\varphi_{\lambda}, \lambda \in P_{+}^{+}$.

Let $\mathcal{L}_{0}^{2}(F)$ denote the Hilbert space of functions on the fundamental domain $F$, which vanish on the boundary $\partial F$ of the fundamental domain, with the scalar product

$$
\left\langle f_{1}, f_{2}\right\rangle=\int_{F} f_{1}(x) \overline{f_{2}(x)} d x .
$$

The set of continuous functions on $F$ (vanishing on the boundary $\partial F$ ) with continuous derivatives is dense in $\mathcal{L}_{0}^{2}(F)$. Therefore, the formulas (10.3)-(10.5) show that the set of orbit functions $\varphi_{\lambda}$, $\lambda \in P_{+}^{+}$, form an orthogonal basis of $\mathcal{L}_{0}^{2}(F)$.

\subsection{Symmetric and antisymmetric multivariate sine and cosine series}

Symmetric and antisymmetric orbit functions for the Coxeter-Dynkin diagram $C_{n}$ can be expressed in terns of symmetric and antisymmetric multivariate sine and cosine functions (see formulas (4.19) and (5.4)). Their application in the formulas for symmetric and antisymmetric orbit function transforms gives antisymmetric and symmetric multivariate sine and cosine series expansions. 
The formulas (10.3) and (10.4), applied to the case $C_{n}$, determine expansions of functions, given on the fundamental domain

$$
F=\left\{1 / 2>x_{1}>x_{2}>\cdots>x_{n}>0\right\}
$$

for the Coxeter-Dynkin diagram $C_{n}$, into antisymmetric multivariate sine functions:

$$
f(x)=\sum_{\mathbf{m} \in P_{+}^{+}} c_{\mathbf{m}} \operatorname{det}\left(\sin 2 \pi m_{i} x_{j}\right)_{i, j=1}^{n},
$$

where $\mathbf{m}=\left(m_{1}, m_{2}, \ldots, m_{n}\right)$ are integer $n$-tuples such that $m_{1}>m_{2}>\cdots>m_{n}>0$, and the coefficients $c_{\mathbf{m}}$ are given by the formula

$$
c_{\mathbf{m}}=2^{2 n} \int_{F} f(x) \operatorname{det}\left(\sin 2 \pi m_{i} x_{j}\right)_{i, j=1}^{n} d x .
$$

The Plancherel formula is of the form

$$
\sum_{\mathbf{m} \in P_{+}^{+}}\left|c_{\mathbf{m}}\right|^{2}=2^{2 n} \int_{F} f(x)|f(x)|^{2} d x .
$$

Similarly, using the symmetric orbit function transform on the fundamental domain $F$ (see Subsection 8.2 in [1]), determined by symmetric orbit functions for the Coxeter-Dynkin diagram $C_{n}$ given by the formulas (4.24) and (5.4), one obtains symmetric multivariate cosine expansion on $F$ :

$$
f(x)=\sum_{\mathbf{m} \in P_{+}} c_{\mathbf{m}} \operatorname{det}^{+}\left(\cos 2 \pi m_{i} x_{j}\right)_{i, j=1}^{n},
$$

where $\mathbf{m}=\left(m_{1}, m_{2}, \ldots, m_{n}\right)$ are integer $n$-tuples such that $m_{1} \geq m_{2} \geq \cdots \geq m_{n} \geq 0$, and the coefficients $c_{\mathbf{m}}$ are given by the formula

$$
c_{\mathbf{m}}=2^{2 n} \int_{F} f(x) \operatorname{det}^{+}\left(\cos 2 \pi m_{i} x_{j}\right)_{i, j=1}^{n} d x .
$$

The Plancherel formula is of the form

$$
\sum_{\mathbf{m} \in P_{+}}\left|c_{\mathbf{m}}\right|^{2}=2^{2 n} \int_{F} f(x)|f(x)|^{2} d x .
$$

\subsection{Orbit function transform on the dominant Weyl chamber}

The expansion (10.3) of functions on the fundamental domain $F$ is an expansion in the antisymmetric orbit functions $\varphi_{\lambda}(x)$ with integral strictly dominant weights $\lambda$. The antisymmetric orbit functions $\varphi_{\lambda}(x)$ with $\lambda$ lying in the dominant Weyl chamber (and not obligatory integral) are not invariant with respect to the corresponding affine Weyl group $W^{\text {aff }}$. They are invariant only with respect to the Weyl group $W$. A fundamental domain of $W$ coincides with the dominant Weyl chamber $D_{+}$. For this reason, the orbit functions $\varphi_{\lambda}(x), \lambda \in D_{+}^{+}$, determine another orbit function transform (a transform on $D_{+}$).

We began with the usual Fourier transforms on $\mathbb{R}^{n}$ :

$$
\begin{aligned}
\tilde{f}(\lambda) & =\int_{\mathbb{R}^{n}} f(x) e^{2 \pi \mathrm{i}\langle\lambda, x\rangle} d x, \\
f(x) & =\int_{\mathbb{R}^{n}} \tilde{f}(\lambda) e^{-2 \pi \mathrm{i}\langle\lambda, x\rangle} d \lambda .
\end{aligned}
$$


Let the function $f(x)$ be anti-invariant with respect to the Weyl group $W$, that is, $f(w x)=$ $(\operatorname{det} w) f(x), w \in W$. It is easy to check that the function $\tilde{f}(\lambda)$ is also anti-invariant with respect to the Weyl group $W$. Replace in (10.10) $\lambda$ by $w \lambda, w \in W$, multiply both sides by det $w$, and sum these both side over $w \in W$. Then instead of (10.10) we obtain

$$
\tilde{f}(\lambda)=\int_{D_{+}} f(x) \varphi_{\lambda}(x) d x, \quad \lambda \in D_{+}^{+}
$$

where we have taken into account that $f(x)$ is anti-invariant with respect to $W$.

Similarly, starting from (10.11), we obtain the inverse formula:

$$
f(x)=\int_{D_{+}} \tilde{f}(\lambda) \overline{\varphi_{\lambda}(x)} d \lambda
$$

For the transforms (10.12) and (10.13) the Plancherel formula

$$
\int_{D_{+}}|f(x)|^{2} d x=\int_{D_{+}}|\tilde{f}(\lambda)|^{2} d \lambda
$$

holds.

\subsection{Symmetric and antisymmetric multivariate sine and cosine integral transforms}

The orbit function transforms (10.12) and (10.13) in the case of the Coxeter-Dynkin diagram $C_{n}$ lead to symmetric and antisymmetric multivariate sine and cosine integral transforms.

Taking into account the expression (4.19) for antisymmetric orbit functions for $C_{n}$ we obtain the transform

$$
\tilde{f}(\lambda)=\int_{D_{+}} f(x) \operatorname{det}\left(\sin 2 \pi \lambda_{i} x_{j}\right)_{i, j=1}^{n} d x
$$

where $\lambda=\left(\lambda_{1}, \lambda_{2}, \ldots, \lambda_{n}\right) \in D_{+}^{+}$, that is, $\lambda_{1}>\lambda_{2}>\cdots>\lambda_{n}>0$ (the function $\tilde{f}(\lambda)$ vanishes on the boundary of $\left.D_{+}\right)$. The inverse transform is of the form

$$
f(x)=2^{2 n} \int_{D_{+}} \tilde{f}(\lambda) \operatorname{det}\left(\sin 2 \pi \lambda_{i} x_{j}\right)_{i, j=1}^{n} d \lambda
$$

For these transforms the Plancherel formula

$$
\int_{D_{+}}|f(x)|^{2} d x=2^{2 n} \int_{D_{+}}|\tilde{f}(\lambda)|^{2} d \lambda
$$

holds.

Similar transformations hold for symmetric multivariate cosine function:

$$
\begin{aligned}
\tilde{f}(\lambda) & =\int_{D_{+}} f(x) \operatorname{det}^{+}\left(\cos 2 \pi \lambda_{i} x_{j}\right)_{i, j=1}^{n} d x \\
f(x) & =2^{2 n} \int_{D_{+}} \tilde{f}(\lambda) \operatorname{det}^{+}\left(\cos 2 \pi \lambda_{i} x_{j}\right)_{i, j=1}^{n} d \lambda
\end{aligned}
$$

where $\lambda=\left(\lambda_{1}, \lambda_{2}, \ldots, \lambda_{n}\right) \in D_{+}$and the function $\operatorname{det}^{+}\left(\cos \lambda_{i} x_{j}\right)_{i, j=1}^{n}$ is given by formula (5.4). 


\section{Multivariate generalization of the finite Fourier transform and of finite sine and cosine transforms}

Along with the integral Fourier transform there exists a discrete Fourier transform. Similarly, it is possible to introduce a finite orbit function transform, based on antisymmetric orbit functions. It is done in the same way as in the case of symmetric orbit functions in [1] by using the results of the paper [21] (see [31]). We first consider the finite Fourier transform. Then we consider a general theory (appropriate for any connected Coxeter-Dynkin diagram). In the last subsections we give concrete antisymmetric and symmetric generalizations of the finite Fourier transform. In particular, we consider antisymmetric and symmetric multivariate finite Fourier transforms, discrete sine and cosine transforms, and antisymmetric and symmetric multivariate discrete sine and cosine transforms.

\subsection{Finite Fourier transform}

Let us fix a positive integer $N$ and consider the numbers

$$
e_{m n}:=N^{-1 / 2} \exp (2 \pi \mathrm{i} m n / N), \quad m, n=1,2, \ldots, N .
$$

The matrix $\left(e_{m n}\right)_{m, n=1}^{N}$ is unitary, that is,

$$
\sum_{k} e_{m k} \overline{e_{n k}}=\delta_{m n}, \quad \sum_{k} e_{k m} \overline{e_{k n}}=\delta_{m n}
$$

Indeed, according to the formula for a sum of a geometric progression we have

$$
\begin{aligned}
& t^{a}+t^{a+1}+\cdots+t^{a+r}=(1-t)^{-1} t^{a}\left(1-t^{r+1}\right), \quad t \neq 1, \\
& t^{a}+t^{a+1}+\cdots+t^{a+r}=r+1, \quad t=1 .
\end{aligned}
$$

Setting $t=\exp (2 \pi \mathrm{i}(m-n) / N), a=1$ and $r=N-1$, we prove (11.2).

Let $f(n)$ be a function of $n \in\{1,2, \ldots, N\}$. We may consider the transform

$$
\sum_{n=1}^{N} f(n) e_{m n} \equiv N^{-1 / 2} \sum_{n=1}^{N} f(n) \exp (2 \pi \mathrm{i} m n / N)=\tilde{f}(m)
$$

Then due to unitarity of the matrix $\left(e_{m n}\right)_{m, n=1}^{N}$, we express $f(n)$ as a linear combination of conjugates of the functions (11.1):

$$
f(n)=N^{-1 / 2} \sum_{m=1}^{N} \tilde{f}(m) \exp (-2 \pi \mathrm{i} m n / N) .
$$

The function $\tilde{f}(m)$ is a finite Fourier transform of $f(n)$. This transform is a linear map. The formula (11.4) gives an inverse transform. The Plancherel formula

$$
\sum_{m=1}^{N}|\tilde{f}(m)|^{2}=\sum_{n=1}^{N}|f(n)|^{2}
$$

holds for transforms (11.3) and (11.4). This means that the finite Fourier transform preserves the norm introduced in the space of functions on $\{1,2, \ldots, N\}$.

The finite Fourier transform on the $r$-dimensional linear space $E_{r}$ is defined similarly. We again fix a positive integer $N$. Let $\mathbf{m}=\left(m_{1}, m_{2}, \ldots, m_{r}\right)$ be an $r$-tuple of integers such that 
each $m_{i}$ runs over the integers $1,2, \ldots, N$. Then the finite Fourier transform on $E_{r}$ is given by the kernel

$$
e_{\mathbf{m n}}:=e_{m_{1} n_{1}} e_{m_{2} n_{2}} \cdots e_{m_{r} n_{r}}=N^{-r / 2} \exp (2 \pi \mathrm{im} \cdot \mathbf{n} / N),
$$

where $\mathbf{m} \cdot \mathbf{n}=m_{1} n_{1}+m_{2} n_{2}+\cdots+m_{r} n_{r}$. If $F(\mathbf{m})$ is a function of $r$-tuples $\mathbf{m}, m_{i} \in\{1,2, \ldots, N\}$, then the finite Fourier transform of $F$ is given by

$$
\tilde{F}(\mathbf{n})=N^{-r / 2} \sum_{\mathbf{m}} F(\mathbf{m}) \exp (2 \pi \mathbf{i m} \cdot \mathbf{n} / N) .
$$

The inverse transform is

$$
F(\mathbf{m})=N^{-r / 2} \sum_{\mathbf{n}} \tilde{F}(\mathbf{n}) \exp (-2 \pi \mathbf{i m} \cdot \mathbf{n} / N) .
$$

The corresponding Plancherel formula is of the form $\sum_{\mathbf{m}}|F(\mathbf{m})|^{2}=\sum_{\mathbf{n}}|\tilde{F}(\mathbf{n})|^{2}$.

\subsection{W-invariant lattices}

In order to determine an analogue of the finite Fourier transform, based on antisymmetric orbit functions, we need an analogue of the set

$$
\left\{\mathbf{m}=\left\{m_{1}, m_{2}, \ldots, m_{n}\right\} \mid m_{i} \in\{1,2, \ldots, N\}\right\},
$$

used for multidimensional finite Fourier transform. Such a set has to be invariant with respect to the Weyl group $W$ (see [21]).

We know that $Q^{\vee}$ is a discrete $W$-invariant subset of $E_{n}$. Clearly, the set $\frac{1}{m} Q^{\vee}$ is also $W$-invariant, where $m$ is a fixed positive integer. Then the set

$$
T_{m}=\frac{1}{m} Q^{\vee} / Q^{\vee}
$$

is finite and $W$-invariant. If $\alpha_{1}, \alpha_{2}, \ldots, \alpha_{l}$ is the set of simple root for the Weyl group $W$, then $T_{m}$ can be identified with the set of elements

$$
m^{-1} \sum_{i=1}^{l} d_{i} \alpha_{i}^{\vee}, \quad d_{i}=0,1,2, \ldots, m-1 .
$$

We select from $T_{m}$ the set of elements which belongs to the fundamental domain $\bar{F}$. These elements lie in the collection $\frac{1}{m} Q^{\vee} \cap \bar{F}$.

Let $\mu \in \frac{1}{m} Q^{\vee} \cap \bar{F}$ be an element determining an element of $T_{m}$ and let $M$ be the least positive integer such that $M \mu \in P^{\vee}$. Then there exists the least positive integer $N$ such that $N \mu \in Q^{\vee}$. One has $M \mid N$ and $N \mid m$.

The collection of points of $T_{m}$ which belong to $F$ (we denote the set of these points by $F_{M}$ ) coincides with the set of elements

$$
s=\frac{s_{1}}{M} \omega_{1}^{\vee}+\cdots+\frac{s_{l}}{M} \omega_{l}^{\vee}, \quad \omega_{i}^{\vee}=\frac{2 \omega_{i}}{\left\langle\alpha_{i}, \alpha_{i}\right\rangle},
$$

where $s_{1}, s_{2}, \ldots, s_{l}$ runs over values from $\{0,1,2, \ldots\}$ and satisfy the following condition: there exists a non-negative integer $s_{0}$ such that

$$
s_{0}+\sum_{i=1}^{l} s_{i} m_{i}=M,
$$


where $m_{1}, m_{2}, \ldots, m_{l}$ are positive integers from formula (2.8). Values of $m_{i}$ for all simple Lie algebras can be found in Subsection 2.4.

Indeed, the fundamental domain consists of all points $y$ from the dominant Weyl chamber for which $\langle y, \xi\rangle \leq 1$, where $\xi$ is the highest (long) root, $\xi=\sum_{i=1}^{l} m_{i} \alpha_{i}$. Since for elements $s$ of (11.6) one has $s_{i} / M \geq 0$ and

$$
\langle s, \xi\rangle=\frac{1}{M} \sum_{i=1}^{l} s_{i} m_{i}=\frac{1}{M}\left(M-s_{0}\right) \leq 1,
$$

then $s \in \bar{F}$. The converse reasoning shows that points of $\frac{1}{m} Q^{\vee} \cap \bar{F}$ must be of the form (11.6).

To every positive integer $M$ there corresponds the grid $F_{M}$ of points (11.6) in $\bar{F}$ which corresponds to some set $T_{n}$ such that $M \mid m$. The precise relation between $M$ and $n$ can be defined by the grid $F_{M}$ (see [21]). Acting upon the grid $F_{M}$ by elements of the Weyl group $W$ we obtain the whole set $T_{m}$.

Since antisymmetric orbit functions vanish on the boundary of a fundamental domain $F$, it make sense to consider also a subgrid $F_{M}^{-}$consisting of all points of $F_{M}$ which do not lie on a wall of $F$.

Example. Grids $F_{M}$ for $A_{1}$. We take into account results of Example in Subsection 4.1. For $A_{1}$ we have $\omega^{\vee}=\omega=\alpha / 2$, where $\alpha$ is the simple root. Elements of $P_{+}$coincide with $m \omega, m \in \mathbb{Z}_{+}$. Fixing $M \in \mathbb{Z}_{+}$we have

$$
F_{M}=\left\{s=\frac{s_{1}}{M}, \text { where } s_{0}+s_{1}=M \text { for } s_{0}, s_{1} \in \mathbb{Z}^{\geq 0}\right\} .
$$

Therefore,

$$
F_{M}=\left\{0, \frac{1}{M}, \frac{2}{M}, \ldots, \frac{M-1}{M}, 1\right\} .
$$

\subsection{Grids $F_{M}$ for $A_{2}, C_{2}$ and $G_{2}$}

In this section we give some examples of grids $F_{M}$ for the rank two cases (see [14] and [15]). Since the long root $\xi$ of $A_{2}$ is representable in the form $\xi=\alpha_{1}+\alpha_{2}$, where $\alpha_{1}$ and $\alpha_{2}$ are simple roots, that is, $m_{1}=m_{2}=1$ (see formula (11.7)), then

$$
F_{M}\left(A_{2}\right)=\left\{\frac{s_{1}}{M} \omega_{1}+\frac{s_{2}}{M} \omega_{2} ; s_{0}+s_{1}+s_{2}=M, s_{0}, s_{1}, s_{2} \in \mathbb{Z}^{\geq 0}\right\} .
$$

It is seen from here that the vertices $0, \omega_{1}, \omega_{2}$ of the fundamental domain $F\left(A_{2}\right)$ belong to each grid $F_{M}\left(A_{2}\right)$. A direct computation shows that in the $\omega$-coordinates we have

$$
\begin{aligned}
& F_{2}\left(A_{2}\right)=\left\{(0,0),(1,0),(0,1),\left(\frac{1}{2}, 0\right),\left(0, \frac{1}{2}\right),\left(\frac{1}{2}, \frac{1}{2}\right)\right\} \\
& F_{3}\left(A_{2}\right)=\left\{(0,0),(1,0),(0,1),\left(\frac{1}{3}, 0\right),\left(0, \frac{1}{3}\right),\left(\frac{2}{3}, 0\right),\left(0, \frac{2}{3}\right),\left(\frac{2}{3}, \frac{1}{3}\right),\left(\frac{1}{3}, \frac{2}{3}\right),\left(\frac{1}{3}, \frac{1}{3}\right)\right\} .
\end{aligned}
$$

In $F_{2}\left(A_{2}\right)$, only the point $\left(\frac{1}{2}, \frac{1}{2}\right)$ does not belong to a wall of the fundamental domain $F\left(A_{2}\right)$. In $F_{3}\left(A_{2}\right)$, three points $\left(\frac{1}{3}, \frac{2}{3}\right),\left(\frac{2}{3}, \frac{1}{3}\right),\left(\frac{1}{3}, \frac{1}{3}\right)$ do not belong to a wall of $F\left(A_{2}\right)$.

The set $F_{5}^{-}\left(A_{2}\right)$ consists of the points

$$
F_{5}^{-}\left(A_{2}\right)=\left\{\left(\frac{1}{5}, \frac{3}{5}\right),\left(\frac{2}{5}, \frac{2}{5}\right),\left(\frac{3}{5}, \frac{1}{5}\right),\left(\frac{1}{5}, \frac{2}{5}\right),\left(\frac{2}{5}, \frac{1}{5}\right),\left(\frac{1}{5}, \frac{1}{5}\right)\right\} .
$$

Since the long root $\xi$ of $C_{2}$ is representable in the form $\xi=2 \alpha_{1}+\alpha_{2}$, where $\alpha_{1}$ and $\alpha_{2}$ are simple roots, that is, $m_{1}=2, m_{2}=1$, then

$$
F_{M}\left(C_{2}\right)=\left\{\frac{s_{1}}{M} \omega_{1}^{\vee}+\frac{s_{2}}{M} \omega_{2}^{\vee} ; s_{0}+2 s_{1}+s_{2}=M, s_{0}, s_{1}, s_{2} \in \mathbb{Z}^{\geq 0}\right\} .
$$


A direct computation shows that in the $\omega^{\vee}$-coordinates we have

$$
\begin{aligned}
& F_{2}\left(C_{2}\right)=\left\{(0,0),(0,1),\left(\frac{1}{2}, 0\right),\left(0, \frac{1}{2}\right)\right\}, \\
& F_{3}\left(C_{2}\right)=\left\{(0,0),(0,1),\left(\frac{1}{3}, 0\right),\left(0, \frac{1}{3}\right),\left(0, \frac{2}{3}\right),\left(\frac{1}{3}, \frac{1}{3}\right)\right\}, \\
& F_{7}^{-}\left(C_{2}\right)=\left\{\left(\frac{1}{7}, \frac{4}{7}\right),\left(\frac{2}{7}, \frac{2}{7}\right),\left(\frac{1}{7}, \frac{3}{7}\right),\left(\frac{2}{7}, \frac{1}{7}\right),\left(\frac{1}{7}, \frac{2}{7}\right),\left(\frac{1}{7}, \frac{1}{7}\right)\right\} .
\end{aligned}
$$

Since the long root $\xi$ of $G_{2}$ is representable in the form $\xi=2 \alpha_{1}+3 \alpha_{2}$, where $\alpha_{1}$ and $\alpha_{2}$ are simple roots, that is, $m_{1}=2, m_{2}=3$, then

$$
F_{M}\left(G_{2}\right)=\left\{\frac{s_{1}}{M} \omega_{1}^{\vee}+\frac{s_{2}}{M} \omega_{2}^{\vee} ; s_{0}+2 s_{1}+3 s_{2}=M, \quad s_{0}, s_{1}, s_{2} \in \mathbb{Z}^{\geq 0}\right\} .
$$

A computation shows that in the $\omega^{\vee}$-coordinates we have

$$
\begin{aligned}
F_{2}\left(G_{2}\right)= & \{(0,0),(1,0)\}, \\
F_{3}\left(G_{2}\right)= & \left\{(0,0),\left(0, \frac{1}{3}\right),\left(\frac{1}{3}, 0\right)\right\}, \\
F_{4}\left(G_{2}\right)= & F_{2}\left(G_{2}\right) \cup\left\{\left(\frac{1}{4}, 0\right),\left(0, \frac{1}{4}\right)\right\}, \\
F_{5}\left(G_{2}\right)= & \left\{(0,0),\left(0, \frac{1}{5}\right),\left(\frac{1}{5}, 0\right),\left(\frac{1}{5}, \frac{1}{5}\right),\left(\frac{2}{5}, 0\right)\right\}, \\
F_{8}\left(G_{2}\right)= & F_{4}\left(G_{2}\right) \cup\left\{\left(\frac{1}{8}, 0\right),\left(0, \frac{1}{8}\right),\left(\frac{1}{8}, \frac{1}{8}\right),\left(\frac{1}{4}, \frac{1}{8}\right)\right\}, \\
F_{14}^{-}\left(G_{2}\right)= & \left\{\left(\frac{1}{7}, \frac{3}{14}\right),\left(\frac{5}{14}, \frac{1}{14}\right),\left(\frac{3}{14}, \frac{1}{7}\right),\left(\frac{1}{14}, \frac{3}{14}\right),\left(\frac{2}{7}, \frac{1}{14}\right),\left(\frac{1}{7}, \frac{1}{7}\right),\right. \\
& \left.\left(\frac{3}{14}, \frac{1}{14}\right),\left(\frac{1}{14}, \frac{1}{7}\right),\left(\frac{1}{7}, \frac{1}{14}\right),\left(\frac{1}{14}, \frac{1}{14}\right)\right\} .
\end{aligned}
$$

\subsection{Expanding in antisymmetric orbit functions through finite sets}

Let us give an analogue of the finite Fourier transform when instead of exponential functions we use antisymmetric orbit functions. This analogue is not so simple as finite Fourier transform. For this reason, we consider some weak form of the transform. In fact, we consider this weak form in order to be able to recover (at least approximately) the decomposition $f(x)=\sum_{\lambda} a_{\lambda} \varphi_{\lambda}(x)$ by values of $f(x)$ on a finite set of points.

Considering the finite Fourier transform in Section 11.1, we have restricted the exponential function to a discrete set. Similarly, in order to determine a finite transform, based on antisymmetric orbit functions, we have to restrict orbit functions $\varphi_{\lambda}(x)$ to appropriate finite sets of values of $x$. Candidates for such finite sets are sets $T_{m}$. However, antisymmetric orbit functions $\varphi_{\lambda}(x)$ with integral $\lambda$ are invariant with respect to the affine Weyl group $W^{\text {aff }}$. For this reason, we consider orbit functions $\varphi_{\lambda}(x)$ on grids $F_{M}$.

On the other side, we also have to choose a finite number of antisymmetric orbit functions, that is, a finite number of integral strictly dominant elements $\lambda$. The best choice is when a number of orbit functions coincides with $\left|F_{M}\right|$. These antisymmetric orbit functions must be selected in such a way that the matrix

$$
\left(\varphi_{\lambda_{i}}\left(x_{j}\right)\right)_{\lambda_{i} \in \Omega, x_{j} \in F_{M}}
$$

(where $\Omega$ is our finite set of strictly dominant elements $\lambda$ ) is not singular. In order to have nonsingularity of this matrix some conditions must be satisfied. In general, they are not known. For this reason, we consider some, more weak, form of the transform (when $|\Omega| \geq\left|F_{M}\right|$ ) and then explain how the set $|\Omega|$ of $\lambda \in P_{+}^{+}$can be chosen in such a way that $|\Omega|=\left|F_{M}\right|$.

Let $O(\lambda)$ and $O(\mu)$ be two different $W$-orbits for integral strictly dominant elements $\lambda$ and $\mu$. We say that the group $T_{m}$ separates $O(\lambda)$ and $O(\mu)$ if for any two elements $\lambda_{1} \in O(\lambda)$ and $\mu_{1} \in O(\mu)$ there exists an element $x \in T_{m}$ such that $\exp \left(2 \pi \mathrm{i}\left\langle\lambda_{1}, x\right\rangle\right) \neq \exp \left(2 \pi \mathrm{i}\left\langle\mu_{1}, x\right\rangle\right)$ (we use here orbits, not signed orbits, since signs of points are not important for this reasoning). Note that $\lambda$ may coincide with $\mu$. 
Let $f_{1}$ and $f_{2}$ be two functions on $E_{n}$ which are finite linear combinations of orbit functions. We introduce a $T_{m}$-scalar product for them by the formula

$$
\left\langle f_{1}, f_{2}\right\rangle_{T_{m}}=\sum_{x \in T_{m}} f_{1}(x) \overline{f_{2}(x)} .
$$

Then the following proposition is true (see [21] and [31]):

Proposition 17. If $T_{m}$ separates the orbits $O(\lambda)$ and $O(\mu), \lambda, \mu \in P_{+}^{+}$, then

$$
\left\langle\varphi_{\lambda}, \varphi_{\mu}\right\rangle_{T_{m}}=m^{n}|W| \delta_{\lambda \mu}
$$

Proof. We have

$$
\begin{aligned}
\left\langle\varphi_{\lambda}, \varphi_{\mu}\right\rangle_{T_{m}} & =\sum_{x \in T_{m}} \sum_{w \in W} \sum_{w^{\prime} \in W}(\operatorname{det} w)\left(\operatorname{det} w^{\prime}\right) \exp \left(2 \pi \mathrm{i}\left\langle w \lambda-w^{\prime} \mu, x\right\rangle\right) \\
& =\sum_{w \in W} \sum_{w^{\prime} \in W}\left(\operatorname{det} w w^{\prime}\right)\left(\sum_{x \in T_{m}} \exp \left(2 \pi \mathrm{i}\left\langle w \lambda-w^{\prime} \mu, x\right\rangle\right)\right) .
\end{aligned}
$$

Since $T_{m}$ separates $O(\lambda)$ and $O(\mu)$, then none of the differences $w \lambda-w^{\prime} \mu$ in the last sum vanishes on $T_{m}$. Since $T_{m}$ is a group, one has

$$
\sum_{x \in T_{m}} \exp \left(2 \pi \mathrm{i}\left\langle w \lambda-w^{\prime} \mu, x\right\rangle\right)=m^{n} \delta_{w \lambda, w^{\prime} \mu}
$$

Therefore, $\left\langle\varphi_{\lambda}, \varphi_{\mu}\right\rangle_{T_{m}}=m^{n}|W| \delta_{\lambda \mu}$. Proposition is proved.

Let $f$ be an anti-invariant (with respect to $W^{\text {aff }}$ ) function on $E_{n}$ which is a finite linear combination of antisymmetric orbit functions:

$$
f(x)=\sum_{\lambda_{j} \in P_{+}^{+}} a_{\lambda_{j}} \varphi_{\lambda_{j}}(x) .
$$

Our aim is to determine $f(x)$ by its values on a finite subset of $E_{n}$, namely, on $T_{m}$.

We suppose that $T_{m}$ separate orbits $O\left(\lambda_{j}\right)$ with $\lambda_{j}$ from the right hand side of (11.10). Then taking the $T_{m}$-scalar product of both sides of (11.10) with $\varphi_{\lambda_{j}}$ and using the relation (11.9) we obtain

$$
a_{\lambda_{j}}=\left(m^{n}|W|\right)^{-1}\left\langle f, \varphi_{\lambda_{j}}\right\rangle_{T_{m}}
$$

Let now $s^{(1)}, s^{(2)}, \ldots, s^{(h)}$ be all elements of $\bar{F} \cap \frac{1}{m} Q^{\vee}$, which do not lie on some wall of Weyl chambers. Then

$$
a_{\lambda_{j}}=m^{-n}|W|^{-1} \sum_{x \in T_{m}} f(x) \overline{\varphi_{\lambda_{j}}(x)}=m^{n} \sum_{i=1}^{h} f\left(s^{(i)}\right) \overline{\varphi_{\lambda_{j}}\left(s^{(i)}\right)} .
$$

Thus, the finite number of values $f\left(s^{(i)}\right), i=1,2, \ldots, h$, of the function $f(x)$ determines the coefficients $a_{\lambda_{j}}$ and, therefore, determines the function $f(x)$ on the whole space $E_{l}$.

This means that we can reconstruct a $W^{\text {aff }}$-anti-invariant function $f(x)$ on the whole space $E_{n}$ by its values on the finite set $F_{M}$ under an appropriate value of $M$. Namely, we have to expand this function, taken on $F_{M}$, into the series (11.10) by means of the coefficients $a_{\lambda_{j}}$, determined by formula (11.11), and then to continue analytically the expansion (11.10) to the 
whole fundamental domain $F$ (and, therefore, to the whole space $E_{n}$ ), that is, to consider the decomposition (11.10) for all $x \in E_{n}$.

We have assumed that the function $f(x)$ is a finite linear combination of orbit functions. If $f(x)$ expands into infinite sum of orbit functions, then for applying the above procedure we have to approximate the function $f(x)$ by taking a finite number of terms in this infinite sum and then apply the procedure. That is, in this case we obtain an approximate expression of the function $f(x)$ by using a finite number of its values.

At last, we explain how to choose a set $\Omega$ in formula (11.8). The set $F_{M}$ consists of the points (11.6). This set determines the set of points

$$
\lambda=s_{1} \omega_{1}+s_{2} \omega_{2}+\cdots+s_{n} \omega_{n},
$$

where $s_{1}, s_{2}, \ldots, s_{n}$ run over the same values as for the set $F_{M}$. The subset of this set, consisting of strictly dominant elements, can be taken as the set $\Omega$ (see [31]).

\subsection{Antisymmetric and symmetric multivariate discrete Fourier transforms}

The discrete Fourier transform of Subsection 11.1 can be generalized to the $n$-dimensional case in a symmetric or antisymmetric form without using the results of Subsection 11.4.

We take the discrete exponential function (11.1),

$$
e_{m}(s):=N^{-1 / 2} \exp (2 \pi \mathrm{i} m s), \quad s \in F_{N} \equiv\left\{\frac{1}{N}, \frac{2}{N}, \ldots, \frac{N-1}{N}, 1\right\}, \quad m \in \mathbb{Z}^{\geq 0},
$$

and make a multivariate discrete exponential function taking a product of $n$ copies of functions (11.1):

$$
\begin{array}{rlr}
\operatorname{EXP}_{\mathbf{m}}(\mathbf{s}): & =e_{m_{1}}\left(s_{1}\right) e_{m_{2}}\left(s_{2}\right) \cdots e_{m_{n}}\left(s_{n}\right) \\
& =N^{-n / 2} \exp \left(2 \pi \mathrm{i} m_{1} s_{1}\right) \exp \left(2 \pi \mathrm{i} m_{2} s_{2}\right) \cdots \exp \left(2 \pi \mathrm{i} m_{n} s_{n}\right), \quad s_{j} \in F_{N}, \quad m_{i} \in \mathbb{Z}^{\geq 0},
\end{array}
$$

where $\mathbf{s}=\left(s_{1}, s_{2}, \ldots, s_{n}\right)$ and $\mathbf{m}=\left(m_{1}, m_{2}, \ldots, m_{n}\right)$. Now we take these multivariate functions for integers $m_{i}$ such that $m_{1}>m_{2}>\cdots>m_{n} \geq 0$ and make an antisymmetrization. As a result, we obtain a finite version of the antisymmetric orbit function (4.14):

$$
e_{\mathbf{m}}(\mathbf{s}):=\left|S_{n}\right|^{-1 / 2} \operatorname{det}\left(e_{m_{i}}\left(s_{j}\right)\right)_{i, j=1}^{n},
$$

where $\left|S_{n}\right|$ is the order of the symmetric group $S_{n}$.

The $n$-tuples $\mathbf{s}$ in (11.13) runs over $F_{N}^{n} \equiv F_{N} \times \cdots \times F_{N}$ ( $n$ times). We denote by $\hat{F}_{N}^{n}$ the subset of $F_{N}^{n}$ consisting of $\mathbf{s} \in F_{N}^{n}$ such that

$$
s_{1}>s_{2}>\cdots>s_{n} \text {. }
$$

Note that acting by the permutations $w \in S_{n}$ upon $\hat{F}_{N}^{n}$ we obtain the whole set $F_{N}^{n}$ without those points which are invariant under some nontrivial permutation $w \in S_{n}$. Clearly, the function (11.13) vanishes on the last points.

Since the discrete exponential functions $e_{m}(s)$ satisfy the equality $e_{m}(s)=e_{m+N}(s)$, we do not need to consider them for all values $m \in \mathbb{Z}^{\geq 0}$. It is enough to consider them for $m \in\{1,2, \ldots, N\}$. By $D_{N}^{+}$we denote the set of integer $n$-tuples $\mathbf{m}=\left(m_{1}, m_{2}, \ldots, m_{n}\right)$ such that

$$
N \geq m_{1}>m_{2}>\cdots>m_{n}>0 .
$$

We need a scalar product in the space of linear combinations of the functions (11.12). It can be given by the formula

$$
\begin{aligned}
\left\langle\operatorname{EXP}_{\mathbf{m}}(\mathbf{s}), \operatorname{EXP}_{\mathbf{m}^{\prime}}(\mathbf{s})\right\rangle \equiv & \prod_{i=1}^{n}\left\langle e_{m_{i}}\left(s_{i}\right), e_{m_{i}^{\prime}}\left(s_{i}\right)\right\rangle:=\prod_{i=1}^{n} \sum_{s_{i} \in F_{N}} e_{m_{i}}\left(s_{i}\right) \overline{e_{m_{i}^{\prime}}\left(s_{i}\right)}=\delta_{\mathbf{m m}^{\prime}} \\
& m_{i}, m_{i}^{\prime} \in\{1,2, \ldots, N\}
\end{aligned}
$$

where we used the relation (11.2). 
Proposition 18. For $\mathbf{m}, \mathbf{m}^{\prime} \in D_{N}^{+}$the discrete functions (11.13) satisfy the orthogonality relation

$$
\left\langle e_{\mathbf{m}}(\mathbf{s}), e_{\mathbf{m}^{\prime}}(\mathbf{s})\right\rangle=\left|S_{n}\right| \sum_{\mathbf{s} \in \hat{F}_{N}^{n}} e_{\mathbf{m}}(\mathbf{s}) \overline{e_{\mathbf{m}^{\prime}}(\mathbf{s})}=\delta_{\mathbf{m m}^{\prime}}
$$

where the scalar product is determined by formula (11.14).

Proof. Since $m_{1}>m_{2}>\cdots>m_{n}>0$, then due to the definition of the scalar product we have

$$
\begin{aligned}
\left\langle e_{\mathbf{m}}(\mathbf{s}), e_{\mathbf{m}^{\prime}}(\mathbf{s})\right\rangle & =\sum_{\mathbf{s} \in F_{N}^{n}} e_{\mathbf{m}}(\mathbf{s}) \overline{e_{\mathbf{m}^{\prime}}(\mathbf{s})} \\
& =\left|S_{n}\right|^{-1} \sum_{w \in S_{n}} \prod_{i=1}^{n} \sum_{s_{i} \in F_{N}} e_{m_{w(i)}}\left(s_{i}\right) \overline{e_{m_{w(i)}^{\prime}}\left(s_{i}\right)}=\delta_{\mathbf{m m}^{\prime}},
\end{aligned}
$$

where $\left(m_{w(1)}, m_{w(2)}, \ldots, m_{w(n)}\right)$ is obtained from $\left(m_{1}, m_{2}, \ldots, m_{n}\right)$ by action by the permutation $w \in S_{n}$. Since functions $e_{\mathbf{m}}(\mathbf{s})$ are antisymmetric with respect to $S_{n}$, then

$$
\sum_{\mathbf{s} \in F_{N}^{n}} e_{\mathbf{m}}(\mathbf{s}) \overline{e_{\mathbf{m}^{\prime}}(\mathbf{s})}=\left|S_{n}\right| \sum_{\mathbf{s} \in \hat{F}_{N}^{n}} e_{\mathbf{m}}(\mathbf{s}) \overline{e_{\mathbf{m}^{\prime}}(\mathbf{s})},
$$

where we have taken into account that $e_{\mathbf{m}}(\mathbf{s})$ vanishes on those $\mathbf{s} \in F_{N}^{n}$ for which there exists $w \in S_{n}, w \neq 1$, such that $w \mathbf{s}=\mathbf{s}$. This proves the proposition.

Let $f$ be a function on $\hat{F}_{N}^{n}$ (or an antisymmetric function on $F_{N}^{n}$ ). Then it can be expanded in the functions (11.13) as

$$
f(\mathbf{s})=\sum_{\mathbf{m} \in D_{N}^{+}} a_{\mathbf{m}} e_{\mathbf{m}}(\mathbf{s})
$$

The coefficients $a_{\mathbf{m}}$ are determined by the formula

$$
a_{\mathbf{m}}=\left|S_{n}\right| \sum_{\mathbf{m} \in \hat{F}_{N}^{n}} f(\mathbf{s}) \overline{e_{\mathbf{m}}(\mathbf{s})}
$$

The expansions (11.17) and (11.18) follow from the facts that numbers of elements in $D_{N}^{+}$and in $\hat{F}_{N}^{n}$ are the same, and that the matrix

$$
\left(e_{\mathbf{m}}(\mathbf{s})\right)_{\mathbf{m} \in D_{N}^{+}, \mathbf{s} \in \hat{F}_{N}^{n}}
$$

is unitary. We call expansions (11.17) and (11.18) antisymmetric multivariate discrete Fourier transforms.

Let us also give a symmetric multivariate discrete Fourier transforms. For this we take the multivariate exponential functions (11.12) for integers $m_{i}$ such that

$$
N \geq m_{1} \geq m_{2} \geq \cdots \geq m_{n} \geq 1
$$

and make a symmetrization. We obtain a finite version of the symmetric orbit function (6.11) in [1] for the case $A_{n}$ :

$$
E_{\mathbf{m}}(\mathbf{s}):=\left|S_{n}\right|^{-1 / 2} \operatorname{det}^{+}\left(e_{m_{i}}\left(s_{j}\right)\right)_{i, j=1}^{n}:=\left|S_{n}\right|^{-1 / 2} \sum_{w \in S_{n}} \prod_{i=1}^{n} e_{m_{w(i)}}\left(s_{i}\right) .
$$


The $n$-tuples s in (11.19) run over $F_{N}^{n} \equiv F_{N} \times \cdots \times F_{N}$ ( $n$ times). We denote by $\breve{F}_{N}^{n}$ the subset of $F_{N}^{n}$ consisting of $\mathbf{s} \in F_{N}^{n}$ such that

$$
s_{1} \geq s_{2} \geq \cdots \geq s_{n}
$$

Note that acting by the permutations $w \in S_{n}$ upon $\breve{F}_{N}^{n}$ we obtain the whole set $F_{N}^{n}$, where each point, having some coordinates $m_{i}$ coinciding, is repeated several times. Namely, a point $\mathbf{s}$ is contained $\left|S_{\mathbf{s}}\right|$ times in $\left\{w \breve{F}_{N}^{n} ; w \in S_{n}\right\}$, where $S_{\mathbf{s}}$ is the subgroup of $S_{n}$ consisting of elements $w \in S_{n}$ such that $w \mathbf{s}=\mathbf{s}$. The number $\left|S_{\mathbf{s}}\right|$ is called a multiplicity of the point $\mathbf{s}$ in the set $\left\{w \breve{F}_{N}^{n} ; w \in S_{n}\right\}$.

By $\breve{D}_{N}^{+}$we denote the set of integer $n$-tuples $\mathbf{m}=\left(m_{1}, m_{2}, \ldots, m_{n}\right)$ such that

$$
N \geq m_{1} \geq m_{2} \geq \cdots \geq m_{n} \geq 1 .
$$

Proposition 19. For $\mathbf{m}, \mathbf{m}^{\prime} \in \breve{D}_{N}^{+}$the discrete functions (11.19) satisfy the orthogonality relation

$$
\left\langle E_{\mathbf{m}}(\mathbf{s}), E_{\mathbf{m}^{\prime}}(\mathbf{s})\right\rangle=\left|S_{n}\right| \sum_{\mathbf{s} \in \breve{F}_{M}^{n}}\left|S_{\mathbf{s}}\right|^{-1} E_{\mathbf{m}}(\mathbf{s}) \overline{E_{\mathbf{m}^{\prime}}(\mathbf{s})}=\left|S_{\mathbf{m}}\right| \delta_{\mathbf{m m}^{\prime}}
$$

Proof. This proposition is proved in the same way as Proposition 18, but we have to take into account a difference between $\breve{F}_{M}^{n}$ and $\hat{F}_{M}^{n}$. Due to the definition of the scalar product we have

$$
\begin{aligned}
\left\langle E_{\mathbf{m}}(\mathbf{s}), E_{\mathbf{m}^{\prime}}(\mathbf{s})\right\rangle & =\sum_{\mathbf{s} \in F_{N}^{n}} E_{\mathbf{m}}(\mathbf{s}) \overline{E_{\mathbf{m}^{\prime}}(\mathbf{s})} \\
& =\left|S_{n}\right|^{-1}\left|S_{\mathbf{m}}\right| \sum_{w \in S_{n}} \prod_{i=1}^{n} \sum_{s_{i} \in F_{N}} e_{m_{w(i)}}\left(s_{i}\right) \overline{e_{m_{w(i)}^{\prime}}\left(s_{i}\right)} \\
& =\left|S_{\mathbf{m}}\right| \delta_{\mathbf{m m}^{\prime}},
\end{aligned}
$$

where $\left(m_{w(1)}, m_{w(2)}, \ldots, m_{w(n)}\right)$ is obtained from $\left(m_{1}, m_{2}, \ldots, m_{n}\right)$ by action by the permutation $w \in S_{n}$. Here we have taken into account that additional summands appear (with respect to (11.16)) because some summands on the right hand side of (11.19) may coincide.

Since functions $E_{\mathbf{m}}(\mathbf{s})$ are symmetric with respect to $S_{n}$, then

$$
\sum_{\mathbf{s} \in F_{N}^{n}} E_{\mathbf{m}}(\mathbf{s}) \overline{E_{\mathbf{m}^{\prime}}(\mathbf{s})}=\left|S_{n}\right| \sum_{\mathbf{s} \in \breve{F}_{N}^{n}}\left|S_{\mathbf{s}}\right|^{-1} E_{\mathbf{m}}(\mathbf{s}) \overline{E_{\mathbf{m}^{\prime}}(\mathbf{s})}
$$

where we have taken into account that under action by $S_{n}$ upon $\breve{F}_{N}^{n}$ a point $\mathbf{s}$ appears $\left|S_{\mathbf{s}}\right|$ times in $F_{N}^{n}$. This proves the proposition.

Let $f$ be a function on $\breve{F}_{N}^{n}$ (or a symmetric function on $F_{N}^{n}$ ). Then it can be expanded in functions (11.19) as

$$
f(\mathbf{s})=\sum_{\mathbf{m} \in \breve{D}_{N}^{+}} a_{\mathbf{m}} E_{\mathbf{m}}(\mathbf{s}) .
$$

The coefficients $a_{\mathbf{m}}$ are determined by the formula

$$
a_{\mathbf{m}}=\left|S_{n}\right|\left|S_{\mathbf{m}}\right|^{-1} \sum_{\mathbf{m} \in \breve{F}_{N}^{n}}\left|S_{\mathbf{s}}\right|^{-1} f(\mathbf{s}) \overline{E_{\mathbf{m}}(\mathbf{s})} .
$$

The expansions (11.21) and (11.22) follow from the facts that numbers of elements in $\breve{D}_{N}^{+}$ and in $\breve{F}_{N}^{n}$ are the same and from the orhogonality relation (11.20). We call expansions (11.21) and (11.22) symmetric multivariate discrete Fourier transforms. 


\subsection{Discrete sine and cosine transforms}

The grid $F_{M}$ for $A_{1}$ is of the form

$$
F_{M}\left(A_{1}\right)=\left\{0, \frac{1}{M}, \frac{2}{M}, \ldots, \frac{M-1}{M}, 1\right\} .
$$

The points 0 and 1 belong to the boundary of the fundamental domain $F\left(A_{1}\right)$ of $W^{\text {aff }}\left(A_{1}\right)$. Therefore, antisymmetric orbit functions of $A_{1}$ vanish on these points and

$$
F_{M}^{-}\left(A_{1}\right)=\left\{\frac{1}{M}, \frac{2}{M}, \ldots, \frac{M-1}{M}\right\} \quad(M-1 \text { points })
$$

(see Subsection 11.2). Since the antisymmetric orbit functions for $A_{1}$ are of the form $\varphi_{\lambda}(x)=$ $2 \mathrm{i} \sin (\pi m \theta)$ (see Example of Subsection 4.1), these functions on the grid $F_{M}$ are given by

$$
\varphi_{m}(s)=2 \mathrm{i} \sin (\pi m s), \quad s \in F_{M}, \quad m \in \mathbb{Z}^{\geq} .
$$

Since $\varphi_{m}(s)=\varphi_{m+M}(s)$, we consider these discrete functions only for $m \in D_{M}:=\{1,2, \ldots$, $M-1\}$. The orthogonality relation for these functions is of the form

$$
\left\langle\varphi_{m}, \varphi_{m^{\prime}}\right\rangle=\sum_{s \in F_{M}^{-}} \varphi_{m}(s) \overline{\varphi_{m^{\prime}}(s)}=2 M \delta_{m m^{\prime}}, \quad m, m^{\prime} \in D_{M}
$$

(see [22]). They determine the following expansion of functions, given on the grid $F_{M}^{-}$:

$$
f(s)=\sum_{m=1}^{M-1} a_{m} \varphi_{m}(s)
$$

where the coefficients $a_{m}$ are given by

$$
a_{m}=\frac{1}{2 M} \sum_{s \in F_{M}^{-}} f(s) \overline{\varphi_{m}(s)} .
$$

Formulas (11.25) and (11.26) determine the discrete sine transform.

The symmetric orbit functions for $A_{1}$ are of the form $\phi_{\lambda}(x)=2 \cos (\pi m \theta)$. Then these functions on the grid $F_{M}\left(A_{1}\right)$ are

$$
\phi_{m}(s)=2 \cos (\pi m s), \quad s \in F_{M}, \quad m \in\{0,1,2, \ldots, M\} .
$$

The scalar product of these functions is given by

$$
\left\langle\phi_{m}, \phi_{m^{\prime}}\right\rangle=\sum_{s \in F_{M}} c_{s} \phi_{m}(s) \overline{\phi_{m^{\prime}}(s)}=r_{m} M \delta_{m m^{\prime}},
$$

where $r_{m}=4$ for $m=0, M$ and $r_{m}=2$ otherwise, $c_{s}=1 / 2$ for $s=0,1$ and $c_{s}=1$ otherwise.

The functions $\phi_{m}$, given by (11.27), determine an expansion of functions on the grid $F_{M}$ as

$$
f(s)=\sum_{m=0}^{M} b_{m} \phi_{m}(s), \quad s \in F_{M},
$$

where the coefficients $b_{m}$ are given by

$$
b_{m}=r_{m}^{-1} \sum_{s \in F_{M}} c_{s} f(s) \phi_{m}(s) .
$$

Formulas (11.29) and (11.30) determine the discrete cosine transform. 


\subsection{Antisymmetric multivariate discrete sine transforms}

The discrete sine and cosine transforms of the previous subsection can be generalized to the $n$-dimensional case in symmetric or antisymmetric form. In fact, these generalizations are finite antisymmetric orbit function transforms (11.10) and (11.11) for multivariate sine transforms and finite symmetric orbit function transforms (9.11) and (9.12) in [1] for multivariate cosine transforms. However, we give in this subsection a derivation of these multivariate sine and cosine transforms, independent of the previous consideration. We need only the 1-dimensional discrete sine and cosine transforms of the previous subsection.

We take the discrete sine function (11.24) and make a multivariate discrete sine function by multiplying $n$ copies of functions (11.24):

$$
\begin{aligned}
& \operatorname{SIN}_{\mathbf{m}}(\mathbf{s}):=(2 \mathrm{i})^{n} \sin \left(\pi m_{1} s_{1}\right) \sin \left(\pi m_{2} s_{2}\right) \cdots \sin \left(\pi m_{n} s_{n}\right), \\
& s_{j} \in F_{M} \equiv F_{M}\left(A_{1}\right), \quad m_{i} \in D_{M} \equiv\{1,2, \ldots, N-1\},
\end{aligned}
$$

where $\mathbf{s}=\left(s_{1}, s_{2}, \ldots, s_{n}\right)$ and $\mathbf{m}=\left(m_{1}, m_{2}, \ldots, m_{n}\right)$. Now we take these multivariate functions for integers $m_{i}$ such that $M>m_{1}>m_{2}>\cdots>m_{n}>0$ and make antisymmetrization. As a result, we obtain a finite version of the orbit function (4.19):

$$
\varphi_{\mathbf{m}}(\mathbf{s}):=(2 \mathrm{i})^{n}\left|S_{n}\right|^{-1 / 2} \operatorname{det}\left(\sin \pi m_{i} s_{j}\right)_{i, j=1}^{n},
$$

where $\left|S_{n}\right|$ is the order of the symmetric group $\left|S_{n}\right|$. (We have here expressions $\sin \pi m_{i} s_{j}$, not $\sin 2 \pi m_{i} s_{j}$ as in (4.19). Note that in (4.17) $m_{i}, i=1,2, \ldots, n$, run over integers and half-integers, whereas in (11.32) $m_{i}$ run over integers. Thus, in fact we have replaced $2 m_{i}$ with half-integer values of $m_{i}$ by $m_{i}$ with integer values of $m_{i}$.)

The $n$-tuple $\mathbf{s}$ in (11.32) runs over $F_{M}^{-}\left(A_{1}\right)^{n} \equiv F_{M}^{-}\left(A_{1}\right) \times \cdots \times F_{M}^{-}\left(A_{1}\right)$ ( $n$ times). We denote by $\hat{F}_{M}^{n}$ the subset of $F_{M}^{-}\left(A_{1}\right)^{n}$ consisting of $\mathbf{s} \in F_{M}^{-}\left(A_{1}\right)^{n}$ such that

$$
s_{1}>s_{2}>\cdots>s_{n}
$$

Note that $s_{i}$ here may take the values $\frac{1}{M}, \frac{2}{M}, \ldots, \frac{M-1}{M}$. Acting by permutations $w \in S_{n}$ upon $\hat{F}_{M}^{n}$ we obtain the whole set $F_{M}^{-}\left(A_{1}\right)^{n}$ without those points which are invariant under some nontrivial permutation $w \in S_{n}$. Clearly, the function (11.32) vanishes on the last points.

We denote by $D_{M}^{+}$the set of integer $n$-tuples $\mathbf{m}=\left(m_{1}, m_{2}, \ldots, m_{n}\right)$ such that

$$
M>m_{1}>m_{2}>\cdots>m_{n}>0 \text {. }
$$

We wish to have a scalar product of functions (11.32). For this we define a scalar product of functions (11.31) as

$$
\left\langle\operatorname{SIN}_{\mathbf{m}}(\mathbf{s}), \operatorname{SIN}_{\mathbf{m}^{\prime}}(\mathbf{s})\right\rangle=\prod_{i=1}^{n}\left\langle\varphi_{m_{i}}\left(s_{i}\right), \varphi_{m_{i}^{\prime}}\left(s_{i}\right)\right\rangle,
$$

where the scalar product $\left\langle\varphi_{m_{i}}\left(s_{i}\right), \varphi_{m_{i}^{\prime}}\left(s_{i}\right)\right\rangle$ is given in Subsection 11.6. Since functions $\varphi_{\mathbf{m}}(\mathbf{s})$ are linear combinations of functions $\operatorname{SIN}_{\mathbf{m}^{\prime}}(\mathbf{s})$, a scalar product for $\varphi_{\mathbf{m}}(\mathbf{s})$ is also defined.

Proposition 20. For $\mathbf{m}, \mathbf{m}^{\prime} \in D_{M}^{+}$, the discrete functions (11.32) satisfy the orthogonality relation

$$
\left\langle\varphi_{\mathbf{m}}(\mathbf{s}), \varphi_{\mathbf{m}^{\prime}}(\mathbf{s})\right\rangle:=\sum_{\mathbf{s} \in F_{M}^{-}\left(A_{1}\right)^{n}} \varphi_{\mathbf{m}}(\mathbf{s}) \overline{\varphi_{\mathbf{m}^{\prime}}(\mathbf{s})}=\left|S_{n}\right| \sum_{\mathbf{s} \in \hat{F}_{M}^{n}} \varphi_{\mathbf{m}}(\mathbf{s}) \overline{\varphi_{\mathbf{m}^{\prime}}(\mathbf{s})}=(2 M)^{n} \delta_{\mathbf{m m}^{\prime}}
$$


Proof. Since $M>m_{1}>m_{2}>\cdots>m_{n}>0$, then due to the orthogonality relation for the sine functions $2 \mathrm{i} \sin (\pi m s)$ (see the previous subsection) we have

$$
\sum_{\mathbf{s} \in F_{M}^{-}\left(A_{1}\right)^{n}} \varphi_{\mathbf{m}}(\mathbf{s}) \overline{\varphi_{\mathbf{m}^{\prime}}(\mathbf{s})}=4^{n}\left|S_{n}\right|^{-1} \sum_{w \in S_{n}} \prod_{i=1}^{n} \sum_{s_{i}=1}^{M-1} \sin \left(\pi m_{w(i)} s_{i}\right) \sin \left(\pi m_{w(i)}^{\prime} s_{i}\right)=(2 M)^{n} \delta_{\mathbf{m m}^{\prime}},
$$

where $\left(m_{w(1)}, m_{w(2)}, \ldots, m_{w(n)}\right)$ is obtained from $\left(m_{1}, m_{2}, \ldots, m_{n}\right)$ by action by the permutation $w \in S_{n}$. Since functions $\varphi_{\mathbf{m}}(\mathbf{s})$ are antisymmetric with respect to $S_{n}$, we have

$$
\sum_{\mathbf{s} \in F_{M}^{-}\left(A_{1}\right)^{n}} \varphi_{\mathbf{m}}(\mathbf{s}) \overline{\varphi_{\mathbf{m}^{\prime}}(\mathbf{s})}=\left|S_{n}\right| \sum_{\mathbf{s} \in \hat{F}_{M}^{n}} \varphi_{\mathbf{m}}(\mathbf{s}) \overline{\varphi_{\mathbf{m}^{\prime}}(\mathbf{s})} .
$$

This proves the proposition.

Let $f$ be a function on $\hat{F}_{M}^{n}$ (or an antisymmetric function on $F_{M}^{-}\left(A_{1}\right)^{n}$ ). Then it can be expanded in functions (11.32) as

$$
f(\mathbf{s})=\sum_{\mathbf{m} \in D_{M}^{+}} a_{\mathbf{m}} \varphi_{\mathbf{m}}(\mathbf{s}),
$$

where the coefficients $a_{\mathbf{m}}$ are determined by the formula

$$
a_{\mathbf{m}}=(2 M)^{-n}\left|S_{n}\right| \sum_{\mathbf{m} \in \hat{F}_{M}^{n}} f(\mathbf{s}) \overline{\varphi_{\mathbf{m}}(\mathbf{s})} .
$$

A validity of the expansions (11.34) and (11.35) follows from the facts that numbers of elements in $D_{M}^{+}$and in $\hat{F}_{M}^{n}$ are the same and from the orthogonality relation (11.33).

\subsection{Symmetric multivariate discrete cosine transforms}

We take the discrete cosine functions (11.27) and make multivariate discrete cosine functions by multiplying $n$ copies of these functions:

$$
\begin{aligned}
& \operatorname{COS}_{\mathbf{m}}(\mathbf{s}):=\phi_{m_{1}}\left(s_{1}\right) \phi_{m_{2}}\left(s_{2}\right) \cdots \phi_{m_{n}}\left(s_{n}\right)=2^{n} \cos \left(\pi m_{1} s_{1}\right) \cos \left(\pi m_{2} s_{2}\right) \cdots \cos \left(\pi m_{n} s_{n}\right), \\
& s_{j} \in F_{M} \equiv F_{M}\left(A_{1}\right), \quad m_{i} \in\{0,1,2, \ldots, M\} .
\end{aligned}
$$

We take these functions for integers $m_{i}$ such that $M \geq m_{1} \geq m_{2} \geq \cdots \geq m_{n} \geq 0$ and make a symmetrization. As a result, we obtain a finite version of the orbit function (4.24):

$$
\phi_{\mathbf{m}}(\mathbf{s}):=2^{n}\left|S_{n}\right|^{-1 / 2} \sum_{w \in S_{n}} \cos \pi m_{w(1)} s_{1} \cdot \cos \pi m_{w(2)} s_{2} \cdots \cos \pi m_{w(n)} s_{n} .
$$

(We have here expressions $\cos \pi m_{i} s_{j}$, not $\cos 2 \pi m_{i} s_{j}$ as in (4.24).)

The $n$-tuple $\mathbf{s}$ in (11.37) runs over $F_{M}^{n} \equiv F_{M}\left(A_{1}\right)^{n}$. We denote by $\breve{F}_{M}^{n}$ the subset of $F_{M}^{n}$ consisting of $\mathbf{s} \in F_{M}^{n}$ such that

$$
s_{1} \geq s_{2} \geq \cdots \geq s_{n}
$$

Note that $s_{i}$ here may take the values $0, \frac{1}{M}, \frac{2}{M}, \ldots, \frac{M-1}{M}, 1$. Acting by permutations $w \in S_{n}$ upon $\breve{F}_{M}^{n}$ we obtain the whole set $F_{M}^{n}$, where points, invariant under some nontrivial permutation $w \in S_{n}$, are repeated several times. It is easy to see that a point $\mathbf{s}_{0} \in F_{M}^{n}$ is repeated $\left|S_{\mathbf{s}_{0}}\right|$ times in the set $\left\{w \breve{F}_{M}^{n} ; w \in S_{n}\right\}$, where $\left|S_{\mathbf{s}_{0}}\right|$ is an order of the subgroup $S_{\mathbf{s}_{0}} \subset S_{n}$, whose elements leaves $\mathbf{s}_{0}$ invariant. 
We denote by $\breve{D}_{M}^{+}$the set of integer $n$-tuples $\mathbf{m}=\left(m_{1}, m_{2}, \ldots, m_{n}\right)$ such that

$$
M \geq m_{1} \geq m_{2} \geq \cdots \geq m_{n} \geq 0 .
$$

A scalar product of functions (11.36) is determined by

$$
\left\langle\operatorname{COS}_{\mathbf{m}}(\mathbf{s}), \operatorname{COS}_{\mathbf{m}^{\prime}}(\mathbf{s})\right\rangle=\prod_{i=1}^{n}\left\langle\phi_{m_{i}}\left(s_{i}\right), \phi_{m_{i}^{\prime}}\left(s_{i}\right)\right\rangle,
$$

where the scalar product $\left\langle\phi_{m_{i}}\left(s_{i}\right), \phi_{m_{i}^{\prime}}\left(s_{i}\right)\right\rangle$ is given by (11.28). Since functions $\phi_{\mathbf{m}}(\mathbf{s})$ are linear combinations of functions $\operatorname{COS}_{\mathbf{m}^{\prime}}(\mathbf{s})$, then a scalar product for $\phi_{\mathbf{m}}(\mathbf{s})$ is also defined.

Proposition 21. For $\mathbf{m}, \mathbf{m}^{\prime} \in \breve{D}_{M}^{+}$, the discrete functions (11.37) satisfy the orthogonality relation

$$
\begin{aligned}
\left\langle\phi_{\mathbf{m}}(\mathbf{s}), \phi_{\mathbf{m}^{\prime}}(\mathbf{s})\right\rangle & =\sum_{\mathbf{s} \in F_{M}^{n}} c_{\mathbf{s}} \phi_{\mathbf{m}}(\mathbf{s}) \overline{\phi_{\mathbf{m}^{\prime}}(\mathbf{s})}=\left|S_{n}\right| \sum_{\mathbf{s} \in \breve{F}_{M}^{n}}\left|S_{\mathbf{s}}\right|^{-1} c_{\mathbf{s}} \phi_{\mathbf{m}}(\mathbf{s}) \overline{\phi_{\mathbf{m}^{\prime}}(\mathbf{s})} \\
& =M^{n} r_{\mathbf{m}}\left|S_{\mathbf{m}}\right| \delta_{\mathbf{m m}^{\prime}},
\end{aligned}
$$

where $c_{\mathbf{s}}=c_{s_{1}} c_{s_{2}} \cdots c_{s_{n}}, r_{\mathbf{s}}=r_{m_{1}} r_{m_{2}} \cdots r_{m_{n}}$, and $c_{s_{i}}$ and $r_{m_{i}}$ are such as in (11.28).

Proof. Due to the orthogonality relation for the cosine functions $\phi_{m}(s)=2 \cos (\pi m s)$ (see formula (11.28)) we have

$$
\begin{aligned}
\sum_{\mathbf{s} \in F_{M}^{n}} c_{\mathbf{s}} \phi_{\mathbf{m}}(\mathbf{s}) \overline{\phi_{\mathbf{m}^{\prime}}(\mathbf{s})} & =4^{n}\left|S_{n}\right|^{-1}\left|S_{\mathbf{m}}\right| \sum_{w \in S_{n}} \prod_{i=1}^{n} \sum_{s_{i}=0}^{M} c_{s_{i}} \cos \left(\pi m_{w(i)} s_{i}\right) \cos \left(\pi m_{w(i)}^{\prime} s_{i}\right) \\
& =\left|S_{\mathbf{m}}\right| M^{n} r_{\mathbf{m}} \delta_{\mathbf{m m}^{\prime}}
\end{aligned}
$$

where $\left(m_{w(1)}, m_{w(2)}, \ldots, m_{w(n)}\right)$ is obtained from $\left(m_{1}, m_{2}, \ldots, m_{n}\right)$ by action by the permutation $w \in S_{n}$. Since functions $\phi_{\mathbf{m}}(\mathbf{s})$ are symmetric with respect to $S_{n}$, we have

$$
\sum_{\mathbf{s} \in F_{M}\left(A_{1}\right)^{n}} c_{\mathbf{s}} \phi_{\mathbf{m}}(\mathbf{s}) \overline{\phi_{\mathbf{m}^{\prime}}(\mathbf{s})}=\left|S_{n}\right| \sum_{\mathbf{s} \in \breve{F}_{M}^{n}}\left|S_{\mathbf{s}}\right|^{-1} c_{\mathbf{s}} \phi_{\mathbf{m}}(\mathbf{s}) \overline{\phi_{\mathbf{m}^{\prime}}(\mathbf{s})}
$$

This proves the proposition.

Let $f$ be a function on $\breve{F}_{M}^{n}$ (or an antisymmetric function on $F_{M}^{n}$ ). Then it can be expanded in functions (11.37) as

$$
f(\mathbf{s})=\sum_{\mathbf{m} \in \breve{D}_{M}^{+}} a_{\mathbf{m}} \phi_{\mathbf{m}}(\mathbf{s})
$$

where the coefficients $a_{\mathbf{m}}$ are determined by the formula

$$
\begin{aligned}
a_{\mathbf{m}} & =M^{-n}\left|S_{\mathbf{m}}\right|^{-1} r_{\mathbf{m}}^{-1}\left\langle f(\mathbf{s}), \phi_{\mathbf{m}}(\mathbf{s})\right\rangle \\
& =M^{-n}\left|S_{\mathbf{m}}\right|^{-1} r_{\mathbf{m}}^{-1}\left|S_{n}\right| \sum_{\mathbf{s} \in \breve{F}_{M}^{n}}\left|S_{\mathbf{s}}\right|^{-1} c_{\mathbf{s}} f(\mathbf{s}) \overline{\phi_{\mathbf{m}}(\mathbf{s})} .
\end{aligned}
$$

A validity of the expansions (11.40) and (11.41) follows from the fact that numbers of elements in $\breve{D}_{M}^{+}$and $\breve{F}_{M}^{n}$ are the same and from the orthogonality relation (11.38). 


\subsection{Other discrete cosine transforms}

Along with the discrete cosine transform of Subsection 11.6 there are other discrete transforms with the discrete cosine function as a kernel. In [34] the discrete cosine transforms are called as DCT-1, DCT-2, DCT-3, DCT-4. The transform DCT-1 is in fact the transform, considered in Subsection 11.6. Let us describe all these transforms (including the transform DCT-1). They are determined by a positive integer $N$.

DCT-1. This transform is given by the kernel

$$
\mu_{r}(k)=\cos \frac{\pi r k}{N}
$$

(we preserve the notation used in the literature on signal processing), where

$$
k, r \in\{0,1,2, \ldots, N\} .
$$

The orthogonality relation for these discrete functions is given by

$$
\sum_{k=0}^{N} c_{k} \cos \frac{\pi r k}{N} \cos \frac{\pi r^{\prime} k}{N}=h_{r} \frac{N}{2} \delta_{r r^{\prime}}
$$

where $c_{k}=1 / 2$ for $k=0, N$ and $c_{k}=1$ otherwise, $h_{r}=2$ for $r=0, N$ and $h_{r}=1$ otherwise.

Thus, these functions give the expansion

$$
f(k)=\sum_{r=0}^{N} a_{r} \cos \frac{\pi r k}{N}, \quad \text { where } \quad a_{r}=\frac{2}{h_{r} N} \sum_{k=0}^{N} c_{k} f(k) \cos \frac{\pi r k}{N} .
$$

DCT-2. This transform is given by the kernel

$$
\omega_{r}(k)=\cos \frac{\pi\left(r+\frac{1}{2}\right) k}{N},
$$

where

$$
k, r \in\{0,1,2, \ldots, N-1\} .
$$

The orthogonality relation for these discrete functions is given by

$$
\sum_{k=0}^{N-1} c_{k} \cos \frac{\pi\left(r+\frac{1}{2}\right) k}{N} \cos \frac{\pi\left(r^{\prime}+\frac{1}{2}\right) k}{N}=\frac{N}{2} \delta_{r r^{\prime}},
$$

where $c_{k}=1 / 2$ for $k=0$ and $c_{k}=1$ otherwise.

These functions determine the expansion

$$
f(k)=\sum_{r=0}^{N-1} a_{r} \omega_{r}(k), \quad \text { where } \quad a_{r}=\frac{2}{N} \sum_{k=0}^{N-1} c_{k} f(k) \omega_{r}(k) .
$$

DCT-3. This transform is determined by the kernel

$$
\sigma_{r}(k)=\cos \frac{\pi r\left(k+\frac{1}{2}\right)}{N},
$$


where $k$ and $r$ run over the values $\{0,1,2, \ldots, N-1\}$. The orthogonality relation for these discrete functions is given by the formula

$$
\sum_{k=0}^{N-1} \cos \frac{\pi r\left(k+\frac{1}{2}\right)}{N} \cos \frac{\pi r^{\prime}\left(k+\frac{1}{2}\right)}{N}=h_{r} \frac{N}{2} \delta_{r r^{\prime}},
$$

where $h_{k}=2$ for $k=0$ and $h_{k}=1$ otherwise.

These functions give the expansion

$$
f(k)=\sum_{r=0}^{N-1} a_{r} \cos \frac{\pi r\left(k+\frac{1}{2}\right)}{N}, \quad \text { where } \quad a_{r}=\frac{2}{h_{r} N} \sum_{k=0}^{N-1} f(k) \cos \frac{\pi r\left(k+\frac{1}{2}\right)}{N} .
$$

DCT-4. This transform is given by the kernel

$$
\tau_{r}(k)=\cos \frac{\pi\left(r+\frac{1}{2}\right)\left(k+\frac{1}{2}\right)}{N},
$$

where $k$ and $r$ run over the values $\{0,1,2, \ldots, N-1\}$. The orthogonality relation for these discrete functions is given by

$$
\sum_{k=0}^{N-1} \cos \frac{\pi\left(r+\frac{1}{2}\right)\left(k+\frac{1}{2}\right)}{N} \cos \frac{\pi\left(r^{\prime}+\frac{1}{2}\right)\left(k+\frac{1}{2}\right)}{N}=\frac{N}{2} \delta_{r r^{\prime}} .
$$

These functions determine the expansion

$$
f(k)=\sum_{r=0}^{N-1} a_{r} \cos \frac{\pi\left(r+\frac{1}{2}\right)\left(k+\frac{1}{2}\right)}{N}
$$

where

$$
a_{r}=\frac{2}{N} \sum_{k=0}^{N-1} f(k) \cos \frac{\pi\left(r+\frac{1}{2}\right)\left(k+\frac{1}{2}\right)}{N} .
$$

Note that there also exist four discrete sine transforms, corresponding to the above discrete cosine transforms. They are obtained from the cosine transforms by replacing in (11.45), (11.48), (11.51) and (11.54) cosines discrete functions by sine discrete functions (see [12] and [35]).

\subsection{Other antisymmetric multivariate discrete cosine transforms}

Each of the discrete cosine transforms DCT-1, DCT-2, DCT-3, DCT-4 generates the corresponding antisymmetric multivariate discrete cosine transforms. We call these transforms as AMDCT-1, AMDCT-2, AMDCT-3 and AMDCT-4. Let us give these transforms without proof. Their proofs are the same as in the case of antisymmetric multivariate discrete cosine transforms of Subsection 11.7. Below we use the notation $D_{N}^{n,-}$ for the subset of the set $D_{N}^{n} \equiv D_{N} \times D_{N} \times$ $\cdots \times D_{N}$ ( $n$ times) with $D_{N}=\{0,1,2, \ldots, N\}$ consisting of points $\mathbf{r}=\left(r_{1}, r_{2}, \ldots, r_{n}\right), r_{i} \in D_{N}$, such that

$$
N \geq r_{1}>r_{2}>\cdots>r_{n} \geq 0 .
$$

AMDCT-1. This transform is given by the kernel

$$
M_{\mathbf{r}}(\mathbf{k})=\left|S_{n}\right|^{-1 / 2} \operatorname{det}\left(\mu_{r_{i}}\left(k_{j}\right)\right)_{i, j=1}^{n},
$$


where $\mu_{r}(k)=\cos \frac{\pi r k}{N}$ and $\mathbf{k}=\left(k_{1}, k_{2}, \ldots, k_{n}\right), k_{i} \in\{0,1,2, \ldots, N\}$. The orthogonality relation for these kernels is

$$
\left\langle M_{\mathbf{r}}(\mathbf{k}), M_{\mathbf{r}^{\prime}}(\mathbf{k})\right\rangle=\left|S_{n}\right| \sum_{\mathbf{k} \in D_{N}^{n,-}} c_{\mathbf{k}} M_{\mathbf{r}}(\mathbf{k}) M_{\mathbf{r}^{\prime}}(\mathbf{k})=h_{\mathbf{r}}\left(\frac{N}{2}\right)^{n} \delta_{\mathbf{r r}^{\prime}}
$$

where

$$
c_{\mathbf{k}}=c_{1} c_{2} \cdots c_{n}, \quad h_{\mathbf{k}}=h_{1} h_{2} \cdots h_{n}
$$

and $c_{i}$ and $h_{j}$ are such as in formula (11.44).

This transform is given by the formula

$$
f(\mathbf{k})=\sum_{\mathbf{r} \in D_{N}^{n,-}} a_{\mathbf{r}} M_{\mathbf{r}}(\mathbf{k}), \quad \text { where } \quad a_{\mathbf{r}}=h_{\mathbf{r}}^{-1}\left|S_{n}\right|\left(\frac{2}{N}\right)^{n} \sum_{\mathbf{k} \in D_{N}^{n,-}} c_{\mathbf{k}} f(\mathbf{k}) M_{\mathbf{r}}(\mathbf{k}) .
$$

The Plancherel formula for this transform is

$$
\left|S_{n}\right| \sum_{\mathbf{k} \in D_{N}^{n,-}} c_{\mathbf{k}}|f(\mathbf{k})|^{2}=\left(\frac{N}{2}\right)^{n} \sum_{\mathbf{r} \in D_{N}^{n,-}} h_{\mathbf{r}}\left|a_{\mathbf{r}}\right|^{2} .
$$

AMDCT-2. We use the subset $D_{N-1}^{n,-}$ of the set $D_{N-1}^{n}$ with $D_{N-1}=\{0,1,2, \ldots, N-1\}$ consisting of points $\mathbf{r}=\left(r_{1}, r_{2}, \ldots, r_{n}\right), r_{i} \in D_{N-1}$, such that

$$
N-1 \geq r_{1}>r_{2}>\cdots>r_{n} \geq 0 .
$$

This transform is given by the kernel

$$
\Omega_{\mathbf{r}}(\mathbf{k})=\left|S_{n}\right|^{-1 / 2} \operatorname{det}\left(\omega_{r_{i}}\left(k_{j}\right)\right)_{i, j=1}^{n}
$$

where $\omega_{r}(k)=\cos \frac{\pi\left(r+\frac{1}{2}\right) k}{N}$ and $\mathbf{k}=\left(k_{1}, k_{2}, \ldots, k_{n}\right), k_{i} \in\{0,1,2, \ldots, N-1\}$. The orthogonality relation for these kernels is

$$
\left\langle\Omega_{\mathbf{r}}(\mathbf{k}), \Omega_{\mathbf{r}^{\prime}}(\mathbf{k})\right\rangle=\left|S_{n}\right| \sum_{\mathbf{k} \in D_{N-1}^{n,-}} c_{\mathbf{k}} \Omega_{\mathbf{r}}(\mathbf{k}) \Omega_{\mathbf{r}^{\prime}}(\mathbf{k})=\left(\frac{N}{2}\right)^{n} \delta_{\mathbf{r r}^{\prime}}
$$

where $c_{\mathbf{k}}=c_{1} c_{2} \cdots c_{n}$ and $c_{i}$ are such as in formula (11.47).

This transform is given by the formula

$$
f(\mathbf{k})=\sum_{\mathbf{r} \in D_{N-1}^{n,-}} a_{\mathbf{r}} \Omega_{\mathbf{r}}(\mathbf{k}), \quad \text { where } \quad a_{\mathbf{r}}=\left|S_{n}\right|\left(\frac{2}{N}\right)^{n} \sum_{\mathbf{k} \in D_{N-1}^{n,-}} c_{\mathbf{k}} f(\mathbf{k}) \Omega_{\mathbf{r}}(\mathbf{k}) .
$$

The Plancherel formula for this transform is of the form

$$
\left|S_{n}\right| \sum_{\mathbf{k} \in D_{N-1}^{n,-}} c_{\mathbf{k}}|f(\mathbf{k})|^{2}=\left(\frac{N}{2}\right)^{n} \sum_{\mathbf{r} \in D_{N-1}^{n,-}}\left|a_{\mathbf{r}}\right|^{2} .
$$

AMDCT-3. This transform is given by the kernel

$$
\Sigma_{\mathbf{r}}(\mathbf{k})=\left|S_{n}\right|^{-1 / 2} \operatorname{det}\left(\sigma_{r_{i}}\left(k_{j}\right)\right)_{i, j=1}^{n}, \quad \mathbf{r} \in D_{N-1}^{n,-}, \quad k_{j} \in D_{N-1},
$$


where $\sigma_{r}(k)=\cos \frac{\pi r\left(k+\frac{1}{2}\right)}{N}$. The orthogonality relation for these kernels is

$$
\left\langle\Sigma_{\mathbf{r}}(\mathbf{k}), \Sigma_{\mathbf{r}^{\prime}}(\mathbf{k})\right\rangle=\left|S_{n}\right| \sum_{\mathbf{k} \in D_{N-1}^{n,-}} \Sigma_{\mathbf{r}}(\mathbf{k}) \Sigma_{\mathbf{r}^{\prime}}(\mathbf{k})=h_{\mathbf{r}}\left(\frac{N}{2}\right)^{n} \delta_{\mathbf{r r}^{\prime}},
$$

where $h_{\mathbf{r}}=h_{1} h_{2} \cdots h_{n}$ and $h_{j}$ are such as in formula (11.50).

This transform is given by the formula

$$
f(\mathbf{k})=\sum_{\mathbf{r} \in D_{N-1}^{n,-}} a_{\mathbf{r}} \Sigma_{\mathbf{r}}(\mathbf{k}), \quad \text { where } \quad a_{\mathbf{r}}=h_{\mathbf{r}}^{-1}\left|S_{n}\right|\left(\frac{2}{N}\right)^{n} \sum_{\mathbf{k} \in D_{N-1}^{n,-}} f(\mathbf{k}) \Sigma_{\mathbf{r}}(\mathbf{k}) .
$$

The Plancherel formula is of the form

$$
\left|S_{n}\right| \sum_{\mathbf{k} \in D_{N-1}^{n,-}}|f(\mathbf{k})|^{2}=\left(\frac{N}{2}\right)^{n} \sum_{\mathbf{r} \in D_{N-1}^{n,-}} h_{\mathbf{r}}\left|a_{\mathbf{r}}\right|^{2} .
$$

AMDCT-4. This transform is given by the kernel

$$
T_{\mathbf{r}}(\mathbf{k})=\left|S_{n}\right|^{-1 / 2} \operatorname{det}\left(\tau_{r_{i}}\left(k_{j}\right)\right)_{i, j=1}^{n}, \quad \mathbf{r} \in D_{N-1}^{n,-}, \quad k_{j} \in D_{N-1},
$$

where $\tau_{r}(k)=\cos \frac{\pi\left(k+\frac{1}{2}\right)\left(r+\frac{1}{2}\right)}{N}$. The orthogonality relation for these kernels is

$$
\left\langle T_{\mathbf{r}}(\mathbf{k}), T_{\mathbf{r}^{\prime}}(\mathbf{k})\right\rangle=\left|S_{n}\right| \sum_{\mathbf{k} \in D_{N-1}^{n,-}} T_{\mathbf{r}}(\mathbf{k}) T_{\mathbf{r}^{\prime}}(\mathbf{k})=\left(\frac{N}{2}\right)^{n} \delta_{\mathbf{r r}^{\prime}} .
$$

This transform is given by the formula

$$
f(\mathbf{k})=\sum_{\mathbf{r} \in D_{N-1}^{n,-}} a_{\mathbf{r}} T_{\mathbf{r}}(\mathbf{k}), \quad \text { where } \quad a_{\mathbf{r}}=\left|S_{n}\right|\left(\frac{2}{N}\right)^{n} \sum_{\mathbf{k} \in D_{N-1}^{n,-}} f(\mathbf{k}) T_{\mathbf{r}}(\mathbf{k}) .
$$

The Plancherel formula for this transform is

$$
\left|S_{n}\right| \sum_{\mathbf{k} \in D_{N-1}^{n,-}}|f(\mathbf{k})|^{2}=\left(\frac{N}{2}\right)^{n} \sum_{\mathbf{r} \in D_{N-1}^{n,-}}\left|a_{\mathbf{r}}\right|^{2} .
$$

\subsection{Other symmetric multivariate discrete cosine transforms}

To each of the discrete cosine transforms DCT-1, DCT-2, DCT-3, DCT-4 there corresponds a symmetric multivariate discrete cosine transform. We denote the corresponding transforms as SMDCT-1, SMDCT-2, SMDCT-3, SMDCT-4. Below we give these transforms without proof (proofs are the same as in the case of symmetric multivariate discrete cosine transforms of Subsection 11.8). We fix a positive integer $N$ and use the notation $D_{N}^{n,+}$ for the subset of the set $D_{N}^{n} \equiv D_{N} \times D_{N} \times \cdots \times D_{N}$ ( $n$ times $)$ with $D_{N}=\{0,1,2, \ldots, N\}$ consisting of points $\mathbf{r}=\left(r_{1}, r_{2}, \ldots, r_{n}\right), r_{i} \in \mathbb{Z}^{\geq 0}$ such that

$$
N \geq r_{1} \geq r_{2} \geq \cdots \geq r_{n} \geq 0
$$

The set $D_{N}^{n,+}$ is an extension of the set $D_{N}^{n,-}$ from the previous subsection by adding points which are invariant with respect of some elements of the permutation group $S_{n}$. 
The set $D_{N}^{n}$ is obtained by action by elements of the group $S_{n}$ upon $D_{N}^{n,+}$, that is, $D_{N}^{n}$ coincides with the set $\left\{w D_{N}^{n,+} ; w \in S_{n}\right\}$. However, in $\left\{w D_{N}^{n,+} ; w \in S_{n}\right\}$, some points occur several times. Namely, a point $\mathbf{k}_{0} \in D_{N}^{n,+}$ occurs $\left|S_{\mathbf{k}_{0}}\right|$ times in the set $\left\{w D_{N}^{n,+} ; w \in S_{n}\right\}$, where $\left|S_{\mathbf{k}_{0}}\right|$ is an order of the subgroup $S_{\mathbf{k}_{0}} \subset S_{n}$ consisting of elements $w \in S_{n}$ leaving $\mathbf{k}_{0}$ invariant.

SMDCT-1. This transform is given by the kernel

$$
\hat{M}_{\mathbf{r}}(\mathbf{k})=\left|S_{n}\right|^{-1 / 2} \sum_{w \in S_{n}} \mu_{r_{w(1)}}\left(k_{1}\right) \mu_{r_{w(2)}}\left(k_{2}\right) \cdots \mu_{r_{w(n)}}\left(k_{n}\right)
$$

where, as before, $\mu_{r}(k)=\cos \frac{\pi r k}{N}$ is the discrete cosine function from Subsection 11.9, $\mathbf{k}=$ $\left(k_{1}, k_{2}, \ldots, k_{n}\right), k_{i} \in\{0,1,2, \ldots, N\}$, and the set $(w(1), w(2), \ldots, w(n))$ is obtained from the set $(1,2, \ldots, n)$ by applying the permutation $w \in S_{n}$. The orthogonality relation for these kernels is

$$
\left\langle\hat{M}_{\mathbf{r}}(\mathbf{k}), \hat{M}_{\mathbf{r}^{\prime}}(\mathbf{k})\right\rangle=\left|S_{n}\right| \sum_{\mathbf{k} \in D_{N}^{n_{+}+}}\left|S_{\mathbf{k}}\right|^{-1} c_{\mathbf{k}} \hat{M}_{\mathbf{r}}(\mathbf{k}) \hat{M}_{\mathbf{r}^{\prime}}(\mathbf{k})=h_{\mathbf{r}}\left(\frac{N}{2}\right)^{n}\left|S_{\mathbf{r}}\right| \delta_{\mathbf{r r}^{\prime}}
$$

where $S_{\mathbf{r}}$ is the subgroup of $S_{n}$ consisting of elements leaving $\mathbf{r}$ invariant,

$$
c_{\mathbf{k}}=c_{1} c_{2} \cdots c_{n}, \quad h_{\mathbf{k}}=h_{1} h_{2} \cdots h_{n}
$$

and $c_{i}$ and $h_{j}$ are such as in formula (11.44).

This transform is given by the formula

$$
f(\mathbf{k})=\sum_{\mathbf{r} \in D_{N}^{n_{+}+}} a_{\mathbf{r}} \hat{M}_{\mathbf{r}}(\mathbf{k})
$$

where

$$
a_{\mathbf{r}}=h_{\mathbf{r}}^{-1}\left|S_{\mathbf{r}}\right|^{-1}\left|S_{n}\right|\left(\frac{2}{N}\right)^{n} \sum_{\mathbf{k} \in D_{N}^{n,+}}\left|S_{\mathbf{k}}\right|^{-1} c_{\mathbf{k}} f(\mathbf{k}) \hat{M}_{\mathbf{r}}(\mathbf{k}) .
$$

The Plancherel formula for this transform is

$$
\left|S_{n}\right| \sum_{\mathbf{k} \in D_{N}^{n,+}}\left|S_{\mathbf{k}}\right|^{-1} c_{\mathbf{k}}|f(\mathbf{k})|^{2}=\left(\frac{N}{2}\right)^{n} \sum_{\mathbf{r} \in D_{N}^{n,+}} h_{\mathbf{r}}\left|S_{\mathbf{r}}\right|\left|a_{\mathbf{r}}\right|^{2} .
$$

This transform is in fact a variation of the symmetric multivariate discrete cosine transforms from Subsection 11.8.

SMDCT-2. This transform is given by the kernel

$$
\begin{aligned}
& \hat{\Omega}_{\mathbf{r}}(\mathbf{k})=\left|S_{n}\right|^{-1 / 2} \sum_{w \in S_{n}} \omega_{r_{w(1)}}\left(k_{1}\right) \omega_{r_{w(2)}}\left(k_{2}\right) \cdots \omega_{r_{w(n)}}\left(k_{n}\right), \\
& \mathbf{r} \in D_{N-1}^{n,+}, \quad r_{j} \in D_{N-1} \equiv\{0,1,2, \ldots, N-1\},
\end{aligned}
$$

where $\omega_{r}(k)=\cos \frac{\pi\left(r+\frac{1}{2}\right) k}{N}$ and $\mathbf{k}=\left(k_{1}, k_{2}, \ldots, k_{n}\right), k_{i} \in\{0,1,2, \ldots, N-1\}$. The orthogonality relation for these kernels is

$$
\left\langle\hat{\Omega}_{\mathbf{r}}(\mathbf{k}), \hat{\Omega}_{\mathbf{r}^{\prime}}(\mathbf{k})\right\rangle=\left|S_{n}\right| \sum_{\mathbf{k} \in D_{N-1}^{n,+}}\left|S_{\mathbf{k}}\right|^{-1} c_{\mathbf{k}} \hat{\Omega}_{\mathbf{r}}(\mathbf{k}) \hat{\Omega}_{\mathbf{r}^{\prime}}(\mathbf{k})=\left(\frac{N}{2}\right)^{n}\left|S_{\mathbf{r}}\right| \delta_{\mathbf{r r}^{\prime}}
$$


where $D_{N-1}^{n,+}$ is the set $D_{N}^{n,+}$ with $N$ replaced by $N-1, c_{\mathbf{k}}=c_{1} c_{2} \cdots c_{n}$ and $c_{j}$ are such as in (11.47).

This transform is given by the formula

$$
f(\mathbf{k})=\sum_{\mathbf{r} \in D_{N-1}^{n,+}} a_{\mathbf{r}} \hat{\Omega}_{\mathbf{r}}(\mathbf{k})
$$

where

$$
a_{\mathbf{r}}=\left|S_{n}\right|\left|S_{\mathbf{r}}\right|^{-1}\left(\frac{2}{N}\right)^{n} \sum_{\mathbf{k} \in D_{N-1}^{n,+}}\left|S_{\mathbf{k}}\right|^{-1} c_{\mathbf{k}} f(\mathbf{k}) \hat{\Omega}_{\mathbf{r}}(\mathbf{k}) .
$$

The Plancherel formula for this transform is of the form

$$
\left|S_{n}\right| \sum_{\mathbf{k} \in D_{N-1}^{n,+}}\left|S_{\mathbf{k}}\right|^{-1} c_{\mathbf{k}}|f(\mathbf{k})|^{2}=\left(\frac{N}{2}\right)^{n} \sum_{\mathbf{r} \in D_{N-1}^{n,+}}\left|S_{\mathbf{r}}\right|\left|a_{\mathbf{r}}\right|^{2} .
$$

SMDCT-3. This transform is given by the kernel

$$
\begin{aligned}
& \hat{\Sigma}_{\mathbf{r}}(\mathbf{k})=\left|S_{n}\right|^{-1 / 2} \sum_{w \in S_{n}} \sigma_{r_{w(1)}}\left(k_{1}\right) \sigma_{r_{w(2)}}\left(k_{2}\right) \cdots \sigma_{r_{w(n)}}\left(k_{n}\right), \\
& \mathbf{r} \in D_{N-1}^{n,+}, \quad r_{j} \in D_{N-1},
\end{aligned}
$$

where $\sigma_{r}(k)=\cos \frac{\pi r\left(k+\frac{1}{2}\right)}{N}$. The orthogonality relation for these kernels is

$$
\left\langle\hat{\Sigma}_{\mathbf{r}}(\mathbf{k}), \hat{\Sigma}_{\mathbf{r}^{\prime}}(\mathbf{k})\right\rangle=\left|S_{n}\right| \sum_{\mathbf{k} \in D_{N-1}^{n_{+}+}}\left|S_{\mathbf{k}}\right|^{-1} \hat{\Sigma}_{\mathbf{r}}(\mathbf{k}) \hat{\Sigma}_{\mathbf{r}^{\prime}}(\mathbf{k})=h_{\mathbf{r}}\left(\frac{N}{2}\right)^{n}\left|S_{\mathbf{r}}\right| \delta_{\mathbf{r r}^{\prime}}
$$

where $h_{\mathbf{r}}=h_{1} h_{2} \cdots h_{n}$ and $h_{i}$ are such as in formula (11.50).

This transform is given by the formula

$$
f(\mathbf{k})=\sum_{\mathbf{r} \in D_{N-1}^{n,+}} a_{\mathbf{r}} \hat{\Sigma}_{\mathbf{r}}(\mathbf{k})
$$

where

$$
a_{\mathbf{r}}=h_{\mathbf{r}}^{-1}\left|S_{\mathbf{r}}\right|^{-1}\left|S_{n}\right|\left(\frac{2}{N}\right)^{n} \sum_{\mathbf{k} \in D_{N-1}^{n,+}}\left|S_{\mathbf{k}}\right|^{-1} f(\mathbf{k}) \hat{\Sigma}_{\mathbf{r}}(\mathbf{k}) .
$$

The Plancherel formula is of the form

$$
\left|S_{n}\right| \sum_{\mathbf{k} \in D_{N-1}^{n,+}}\left|S_{\mathbf{k}}\right|^{-1}|f(\mathbf{k})|^{2}=\left(\frac{N}{2}\right)^{n} \sum_{\mathbf{r} \in D_{N-1}^{n,+}} h_{\mathbf{r}}\left|S_{\mathbf{r}}\right|\left|a_{\mathbf{r}}\right|^{2} .
$$

SMDCT-4. This transform is given by the kernel

$$
\begin{aligned}
& \hat{T}_{\mathbf{r}}(\mathbf{k})=\left|S_{n}\right|^{-1 / 2} \sum_{w \in S_{n}} \tau_{r_{w(1)}}\left(k_{1}\right) \tau_{r_{w(2)}}\left(k_{2}\right) \cdots \tau_{r_{w(n)}}\left(k_{n}\right), \\
& \mathbf{r} \in D_{N-1}^{n,+}, \quad r_{j} \in D_{N-1},
\end{aligned}
$$


where $\tau_{r}(k)=\cos \frac{\pi\left(k+\frac{1}{2}\right)\left(r+\frac{1}{2}\right)}{N}$. The orthogonality relation for these kernels is

$$
\left\langle\hat{T}_{\mathbf{r}}(\mathbf{k}), \hat{T}_{\mathbf{r}^{\prime}}(\mathbf{k})\right\rangle=\left|S_{n}\right| \sum_{\mathbf{k} \in D_{N-1}^{n,+}}\left|S_{\mathbf{k}}\right|^{-1} \hat{T}_{\mathbf{r}}(\mathbf{k}) \hat{T}_{\mathbf{r}^{\prime}}(\mathbf{k})=\left(\frac{N}{2}\right)^{n}\left|S_{\mathbf{r}}\right| \delta_{\mathbf{r r}^{\prime}} .
$$

This transform is given by the formula

$$
f(\mathbf{k})=\sum_{\mathbf{r} \in D_{N-1}^{n,+}} a_{\mathbf{r}} \hat{T}_{\mathbf{r}}(\mathbf{k})
$$

where

$$
a_{\mathbf{r}}=\left(\frac{2}{N}\right)^{n}\left|S_{\mathbf{r}}\right|^{-1}\left|S_{n}\right| \sum_{\mathbf{k} \in D_{N-1}^{n,+}}\left|S_{\mathbf{k}}\right|^{-1} f(\mathbf{k}) \hat{T}_{\mathbf{r}}(\mathbf{k}) .
$$

The Plancherel formula for this transform is

$$
\left|S_{n}\right| \sum_{\mathbf{k} \in D_{N-1}^{n,+}}\left|S_{\mathbf{k}}\right|^{-1}|f(\mathbf{k})|^{2}=\left(\frac{N}{2}\right)^{n} \sum_{\mathbf{r} \in D_{N-1}^{n,+}}\left|S_{\mathbf{r}}\right|\left|a_{\mathbf{r}}\right|^{2}
$$

\section{Solutions of the Laplace equation on $n$-dimensional simplexes}

We have seen in [1] that symmetric orbit functions are solutions of the Neumann boundary value problem on $n$-dimensional simplexes. That is, they are solutions of the Laplace equation $\Delta f(x)=\lambda f(x)$ on the fundamental domain $F$ of the corresponding affine Weyl group $W^{\text {aff }}$ satisfying the Neumann boundary condition

$$
\left.\frac{\partial \phi_{\lambda}(x)}{\partial m}\right|_{\partial F}=0, \quad \lambda \in P_{+}
$$

where $\partial F$ is the $(n-1)$-dimensional boundary of $F$ and $m$ is the normal to the boundary. In this section we show that antisymmetric orbit functions are solutions of the Laplace operator, which vanish on the boundary $\partial F$ of the fundamental domain $F$.

\subsection{The case of $n$-dimensional simplexes related to $A_{n}, B_{n}, C_{n}$ and $D_{n}$}

Let $F$ be the fundamental domain of one of the affine Weyl groups $W^{\text {aff }}\left(A_{n}\right), W^{\text {aff }}\left(B_{n}\right)$, $W^{\text {aff }}\left(C_{n}\right), W^{\text {aff }}\left(D_{n}\right)$ (see Subsection 5.9 for an explicit form of these domains). We use orthogonal coordinates $x_{1}, x_{2}, \ldots, x_{n+1}$ on $F$ in the case of $W^{\text {aff }}\left(A_{n}\right)$ and the orthogonal coordinates $x_{1}, x_{2}, \ldots, x_{n}$ in other cases (see Section 3). Thus the fundamental domain $F$ for $W^{\text {aff }}\left(A_{n}\right)$ is placed in the hyperplane $x_{1}+x_{2}+\cdots+x_{n+1}=0$.

The Laplace operator on $F$ in the orthogonal coordinates has the form

$$
\Delta=\frac{\partial^{2}}{\partial x_{1}^{2}}+\frac{\partial^{2}}{\partial x_{2}^{2}}+\cdots+\frac{\partial^{2}}{\partial x_{r}^{2}}
$$

where $r=n+1$ for $A_{n}$ and $r=n$ for $B_{n}, C_{n}$ and $D_{n}$. Let us consider the case $B_{n}$. We take a summand from the expression (4.16) for the antisymmetric orbit function $\varphi_{\lambda}(x)$ of $B_{n}$ and act upon it by the operator $\Delta$. We get

$$
\Delta e^{2 \pi \mathrm{i}\left((w(\varepsilon \lambda))_{1} x_{1}+\cdots+(w(\varepsilon \lambda))_{n} x_{n}\right)}
$$




$$
\begin{aligned}
& =-4 \pi^{2}\left[\left(\varepsilon_{1} m_{1}\right)^{2}+\cdots+\left(\varepsilon_{n} m_{n}\right)^{2}\right] e^{2 \pi \mathrm{i}\left((w(\varepsilon \lambda))_{1} x_{1}+\cdots+(w(\varepsilon \lambda))_{n} x_{n}\right)} \\
& =-4 \pi^{2}\left(m_{1}^{2}+\cdots+m_{n}^{2}\right) e^{2 \pi \mathrm{i}\left((w(\varepsilon \lambda))_{1} x_{1}+\cdots+(w(\varepsilon \lambda))_{n} x_{n}\right)} \\
& =-4 \pi^{2}\langle\lambda, \lambda\rangle e^{2 \pi \mathrm{i}\left((w(\varepsilon \lambda))_{1} x_{1}+\cdots+(w(\varepsilon \lambda))_{n} x_{n}\right)},
\end{aligned}
$$

where $\lambda=\left(m_{1}, m_{2}, \ldots, m_{n}\right)$ is the weight, determining $\varphi_{\lambda}(x)$, in the orthogonal coordinates and $w \in S_{n}$. Since this action of $\Delta$ does not depend on a summand from (4.16), we have

$$
\Delta \varphi_{\lambda}(x)=-4 \pi^{2}\langle\lambda, \lambda\rangle \varphi_{\lambda}(x) .
$$

For $A_{n}, C_{n}$ and $D_{n}$ this formula also holds and the corresponding proofs are the same. Remark that in the case $A_{n}$ the scalar product $\langle\lambda, \lambda\rangle$ is equal to

$$
\langle\lambda, \lambda\rangle=m_{1}^{2}+m_{2}^{2}+\cdots+m_{n+1}^{2} .
$$

The formula (12.2) can be generalized in the following way. Let $\sigma_{k}\left(y_{1}, y_{2}, \ldots, y_{r}\right)$ be the $k$-th elementary symmetric polynomial of degree $k$ of the variables $y_{1}, y_{2}, \ldots, y_{r}$, that is,

$$
\sigma_{k}\left(y_{1}, y_{2}, \ldots, y_{r}\right)=\sum_{1 \leq k_{1}<k_{2}<\cdots<k_{r} \leq n} y_{k_{1}} y_{k_{2}} \cdots y_{k_{r}} .
$$

Then

$$
\sigma_{k}\left(\frac{\partial^{2}}{\partial x_{1}^{2}}, \frac{\partial^{2}}{\partial x_{2}^{2}}, \ldots, \frac{\partial^{2}}{\partial x_{r}^{2}}\right) \varphi_{\lambda}(x)=\left(-4 \pi^{2}\right)^{k} \sigma_{k}\left(m_{1}^{2}, m_{2}^{2}, \ldots, m_{r}^{2}\right) \varphi_{\lambda}(x), \quad k=1,2, \ldots, r,
$$

where, as before, $r=n+1$ for $A_{n}$ and $r=n$ for $B_{n}, C_{n}$ and $D_{n} . r$ differential equations (12.3) are algebraically independent.

Thus, antisymmetric orbit functions are eigenfunctions of the operator $\sigma_{k}\left(\frac{\partial^{2}}{\partial x_{1}^{2}}, \frac{\partial^{2}}{\partial x_{2}^{2}}, \ldots, \frac{\partial^{2}}{\partial x_{r}^{2}}\right)$, $k=1,2, \ldots, n$, on the fundamental domain $F$ satisfying the boundary condition

$$
\varphi_{\lambda}(x)=0, \quad \lambda \in D_{+},
$$

(see Subsection 5.3).

\subsection{The Laplace operator in $\omega$-basis}

We may parametrize elements of $F$ by coordinates in the $\omega$-basis: $x=\theta_{1} \omega_{1}+\cdots+\theta_{2} \omega_{2}$. Denoting by $\partial_{k}$ partial derivative with respect to $\theta_{k}$, we have the Laplace operator $\Delta$ in the form

$$
\Delta=\frac{1}{2} \sum_{i, j=1}^{n}\left\langle\alpha_{j}, \alpha_{j}\right\rangle^{-1} M_{i j} \partial_{i} \partial_{j},
$$

where $\left(M_{i j}\right)$ is the corresponding Cartan matrix. One can see that it is indeed the Laplace operator as follows. The matrix $\left(S_{i j}\right)=\left(\left\langle\alpha_{j}, \alpha_{j}\right\rangle^{-1} M_{i j}\right)$ is symmetric with respect to transposition and its determinant is positive. Hence it can be diagonalized, so that $\Delta$ becomes a sum of second derivatives with no mixed derivative terms.

\subsection{Rank two and three special cases}

We write down explicit form of the Laplace operators in coordinates in the $\omega$-basis for ranks 2 and 3. For rank two the operator $\Delta$ is of the form

$$
A_{2}:\left(\partial_{1}^{2}-\partial_{1} \partial_{2}+\partial_{2}^{2}\right) \varphi=-\frac{4 \pi^{2}}{3}\left(a^{2}+a b+b^{2}\right) \varphi, \quad F=\left\{0, \omega_{1}, \omega_{2}\right\},
$$




$$
\begin{array}{ll}
C_{2}:\left(2 \partial_{1}^{2}-2 \partial_{1} \partial_{2}+\partial_{2}^{2}\right) \varphi=-2 \pi^{2}\left(a^{2}+4 a b+4 b^{2}\right) \varphi, \quad F=\left\{0, \omega_{1}, \omega_{2}\right\}, \\
G_{2}:\left(\partial_{1}^{2}-3 \partial_{1} \partial_{2}+3 \partial_{2}^{2}\right) \varphi=-\frac{4 \pi^{2}}{3}\left(3 a^{2}+3 a b+b^{2}\right) \varphi, \quad F=\left\{0, \frac{\omega_{1}}{2}, \omega_{2}\right\}
\end{array}
$$

Here, to simplify notation, $\varphi$ stands for $\varphi_{\lambda}(x), \lambda=\left(\begin{array}{ll}a & b\end{array}\right)$ and $x=\left(\theta_{1} \theta_{2}\right)$. Although the same symbols are used for analogous objects in the three cases, their geometric meaning is very different. It is given by the appropriate Cartan matrix $M$ from (2.1). In particular, the vertices of $F$ form an equilateral triangle in the case of $A_{2}$, for $C_{2}$ the triangle is half of a square, and it is a half of an equilateral triangle for $G_{2}$.

In the semisimple case $A_{1} \times A_{1}$ one has $M=2\left(\begin{array}{ll}1 & 0 \\ 0 & 1\end{array}\right)$, therefore $\Delta=2 \partial_{1}^{2}+2 \partial_{2}^{2}$, and $\varphi_{\lambda}(x)$ is the product of two antisymmetric orbit functions, one from each $A_{1}$. The fundamental domain is a square.

There are three 3 -dimensional cases to consider, namely $A_{3}, B_{3}$, and $C_{3}$. In addition there are four cases involving non-simple groups of the same rank. For $A_{3}, B_{3}$, and $C_{3}$ the result can be represented by the formulas

$$
\begin{aligned}
& A_{3}: \quad \Delta=\partial_{1}^{2}+\partial_{2}^{2}+\partial_{3}^{2}-\partial_{1} \partial_{2}-\partial_{2} \partial_{3}, \\
& B_{3}: \quad \Delta=\partial_{1}^{2}+\partial_{2}^{2}+2 \partial_{3}^{2}-\partial_{1} \partial_{2}-2 \partial_{2} \partial_{3}, \\
& C_{3}: \quad \Delta=2 \partial_{1}^{2}+2 \partial_{2}^{2}+2 \partial_{3}^{2}-2 \partial_{1} \partial_{2}-2 \partial_{2} \partial_{3} .
\end{aligned}
$$

\subsection{Antisymmetric orbit functions as eigenfunctions of other operators}

Antisymmetric orbit functions are eigenfunctions of many other operators. We consider examples of such operators.

We associate with each $y \in E_{n}$ the shift operator $T_{y}$ which acts on the exponential functions $e^{2 \pi \mathrm{i}\langle\lambda, x\rangle}$ as

$$
T_{y} e^{2 \pi \mathrm{i}\langle\lambda, x\rangle}=e^{2 \pi \mathrm{i}\langle\lambda, x+y\rangle}=e^{2 \pi \mathrm{i}\langle\lambda, y\rangle} e^{2 \pi \mathrm{i}\langle\lambda, x\rangle} .
$$

The action of elements of the Weyl group $W$ on functions, given on $E_{n}$, is given as $w f(x)=$ $f(w x)$. For each $y \in E_{n}$ we define an operator acting on orbit functions by the formula

$$
D_{y}=\sum_{w \in W}(\operatorname{det} w) w T_{y} .
$$

Then

$$
\begin{aligned}
D_{y} \varphi_{\lambda}(x) & =D_{y} \sum_{w \in W}(\operatorname{det} w) e^{2 \pi \mathrm{i}\langle w \lambda, x\rangle} \\
& =\sum_{w^{\prime} \in W}\left(\operatorname{det} w^{\prime}\right) \sum_{w \in W}(\operatorname{det} w) e^{2 \pi \mathrm{i}\langle w \lambda, y\rangle} e^{2 \pi \mathrm{i}\left\langle w \lambda, w^{\prime} x\right\rangle} \\
& =\sum_{w \in W}(\operatorname{det} w) e^{2 \pi \mathrm{i}\langle w \lambda, y\rangle} \sum_{w^{\prime} \in W}\left(\operatorname{det} w^{\prime}\right) e^{2 \pi \mathrm{i}\left\langle w \lambda, w^{\prime} x\right\rangle} \\
& =\sum_{w \in W}(\operatorname{det} w) e^{2 \pi \mathrm{i}\langle w \lambda, y\rangle} \sum_{w^{\prime} \in W}\left(\operatorname{det} w^{\prime}\right) e^{2 \pi \mathrm{i}\left\langle w^{\prime-1} w \lambda, x\right\rangle} \\
& =\sum_{w \in W} e^{2 \pi \mathrm{i}\langle w \lambda, y\rangle} \sum_{w^{\prime} \in W}\left(\operatorname{det} w^{\prime-1} w\right) e^{2 \pi \mathrm{i}\left\langle w^{\prime-1} w \lambda, x\right\rangle} \\
& =\sum_{w \in W} e^{2 \pi \mathrm{i}\langle w \lambda, y\rangle} \varphi_{\lambda}(x)=\phi_{\lambda}(y) \varphi_{\lambda}(x),
\end{aligned}
$$

that is, $\varphi_{\lambda}(x)$ is an eigenfunction of the operator $D_{y}$ with eigenvalue $\phi_{\lambda}(y)$. 
Now we consider the operator

$$
\hat{D}_{y}=\sum_{w \in W} w T_{y}
$$

Then, conducting the same reasoning as above, we receive the relation

$$
\hat{D}_{y} \varphi_{\lambda}(x)=\varphi_{\lambda}(y) \varphi_{\lambda}(x),
$$

that is, $\varphi_{\lambda}(x)$ is an eigenfunction of the operator $\hat{D}_{y}$ with eigenvalue $\varphi_{\lambda}(y)$.

It is proved in the same way that

$$
D_{y} \phi_{\lambda}(x)=\varphi_{\lambda}(y) \phi_{\lambda}(x)
$$

that is, the symmetric orbit function $\phi_{\lambda}(x)$ is an eigenfunction of the operator $D_{y}$ with eigenvalue $\varphi_{\lambda}(y)$.

It is shown similarly that in the cases of $A_{n}, B_{n}, C_{n}, D_{n}$ antisymmetric orbit functions $\varphi_{\lambda}(x)$ are eigenfunctions of the operators

$$
\sum_{w \in W} w \frac{\partial^{2}}{\partial x_{i}^{2}}, \quad i=1,2, \ldots, r
$$

where $x_{1}, x_{2}, \ldots, x_{r}$ are orthogonal coordinates of the point $x, r=n+1$ for $A_{n}$ and $r=n$ for other cases. In fact, these operators are multiple to the Laplace operator $\Delta$.

It is easy to show that in the cases of $A_{n}, B_{n}, C_{n}$, and also of $D_{n}$ with even $n$, antisymmetric orbit functions $\varphi_{\lambda}(x)$ are solutions of the equations

$$
\sum_{w \in W} w \frac{\partial}{\partial x_{i}} f=0, \quad i=1,2, \ldots, r
$$

\section{Symmetric and antisymmetric functions}

Symmetric (antisymmetric) orbit functions are symmetrized (antisymmetrized) versions of the exponential function, when symmetrization (antisymmetrization) is fulfilled by a Weyl group. Instead of the exponential function we can take any other set of functions, for example, a set of orthogonal polynomials or a countable set of functions. Then we obtain a corresponding set of orthogonal symmetric (antisymmetric) polynomials or a set of symmetric (antisymmetric) functions. Such sets of polynomials and functions are a subject of investigation in this section.

\subsection{Symmetrization and antisymmetrization by (anti)symmetric orbit functions}

Symmetric and antisymmetric orbit functions can be used for symmetrization and antisymmetrization of functions. Let $u_{m}(x), m=0,1,2, \ldots$, be a set of continuous functions of one variables. We create functions of $n$ variables

$$
u_{i_{1}, i_{2}, \ldots, i_{n}}\left(x_{1}, x_{2}, \ldots, x_{n}\right) \equiv u_{i_{1}}\left(x_{1}\right) u_{i_{2}}\left(x_{2}\right) \cdots u_{i_{n}}\left(x_{n}\right), \quad i_{k}=0,1,2, \ldots
$$

Then the functions

$$
\tilde{u}_{i_{1}, i_{2}, \ldots, i_{n}}\left(\lambda_{1}, \lambda_{2}, \ldots, \lambda_{n}\right)=\int_{F} u_{i_{1}, i_{2}, \ldots, i_{n}}\left(x_{1}, x_{2}, \ldots, x_{n}\right) \phi_{\lambda}\left(x_{1}, x_{2}, \ldots, x_{n}\right) d \mathbf{x},
$$


where $\lambda \equiv\left(\lambda_{1}, \lambda_{2}, \ldots, \lambda_{n}\right), \phi_{\lambda}(x)$ is a symmetric orbit function, and $d \mathbf{x}$ is the Euclidean measure on $E_{n}$ (that is, $d \mathbf{x}=d x_{1} \cdots d x_{n}$ ), is symmetric with respect to the action of the Weyl group $W$. Indeed, for $w \in W$ we have

$$
\begin{aligned}
\tilde{u}_{i_{1}, i_{2}, \ldots, i_{n}}(w \lambda) & =\int_{F} u_{i_{1}, i_{2}, \ldots, i_{n}}\left(x_{1}, x_{2}, \ldots, x_{n}\right) \phi_{w \lambda}\left(x_{1}, x_{2}, \ldots, x_{n}\right) d \mathbf{x} \\
& =\int_{F} u_{i_{1}, i_{2}, \ldots, i_{n}}\left(x_{1}, x_{2}, \ldots, x_{n}\right) \phi_{\lambda}\left(x_{1}, x_{2}, \ldots, x_{n}\right) d \mathbf{x}=\tilde{u}_{i_{1}, i_{2}, \ldots, i_{n}}(\lambda) .
\end{aligned}
$$

Similarly, the functions

$$
\hat{u}_{i_{1}, i_{2}, \ldots, i_{n}}\left(\lambda_{1}, \lambda_{2}, \ldots, \lambda_{n}\right)=\int_{F} u_{i_{1}, i_{2}, \ldots, i_{n}}\left(x_{1}, x_{2}, \ldots, x_{n}\right) \varphi_{\lambda}\left(x_{1}, x_{2}, \ldots, x_{n}\right) d \mathbf{x}
$$

where $\varphi_{\lambda}(x)$ is an antisymmetric orbit function, are antisymmetric with respect to the action of the Weyl group $W$. In particular, the functions $\hat{u}_{i_{1}, i_{2}, \ldots, i_{n}}(\lambda)$ vanish on Weyl chambers.

Formulas (13.1) and (13.2) are used for obtaining symmetric and antisymmetric functions or polynomials.

\subsection{Eigenfunctions of (anti)symmetric orbit function transform for $W\left(A_{n}\right)$}

Let $H_{n}(x), n=0,1,2, \ldots$, be the well-known Hermite polynomials. They are defined by the formula

$$
H_{n}(x)=n ! \sum_{m=0}^{[n / 2]} \frac{(-1)^{m}(2 x)^{n-2 m}}{m !(n-2 m) !}
$$

where $[n / 2]$ is an integral part of the number $n / 2$. These polynomials obey the difference equation

$$
\left(\frac{d^{2}}{d x^{2}}-2 x \frac{d}{d x}+2 n\right) H_{n}(x)=0 .
$$

They satisfy the relation

$$
\frac{1}{\sqrt{2 \pi}} \int_{-\infty}^{\infty} e^{\mathrm{i} p x} e^{-p^{2} / 2} H_{m}(p) d p=\mathrm{i}^{-m} e^{-x^{2} / 2} H_{m}(x)
$$

(see, for example, Subsection 12.2.4 in [36]), which can be written in the form

$$
\int_{-\infty}^{\infty} e^{2 \pi \mathrm{i} p x} e^{-\pi p^{2}} H_{m}(\sqrt{2 \pi} p) d p=\mathrm{i}^{-m} e^{-\pi x^{2}} H_{m}(\sqrt{2 \pi} x) .
$$

Using the Hermite polynomials we create polynomials of many variables

$$
H_{\mathbf{m}}(\mathbf{x}) \equiv H_{m_{1}, m_{2}, \ldots, m_{n}}\left(x_{1}, x_{2}, \ldots, x_{n}\right):=H_{m_{1}}\left(x_{1}\right) H_{m_{2}}\left(x_{2}\right) \cdots H_{m_{n}}\left(x_{n}\right) .
$$

The functions

$$
e^{-|\mathbf{x}|^{2} / 2} H_{\mathbf{m}}(\mathbf{x}), \quad m_{i}=0,1,2, \ldots, \quad i=1,2, \ldots, n,
$$

form an orthogonal basis of the Hilbert space $L^{2}\left(\mathbb{R}^{n}\right)$ with the scalar product

$$
\left\langle f_{1}, f_{2}\right\rangle:=\int_{\mathbb{R}^{n}} f_{1}(\mathbf{x}) \overline{f_{2}(\mathbf{x})} d \mathbf{x},
$$

where $d \mathbf{x}=d x_{1} d x_{2} \cdots d x_{n}$. 
The polynomials $H_{\mathbf{m}}(\mathbf{x})$ satisfy the differential equation

$$
\left(\Delta-2 \sum_{i=1}^{n} x_{i} \frac{\partial}{\partial x_{i}}+2|\mathbf{m}|\right) H_{\mathbf{m}}(\mathbf{x})=0,
$$

where $\Delta$ is the Laplace operator $\Delta=\sum_{m=0}^{n} \frac{\partial^{2}}{\partial x_{m}^{2}}$ and $|\mathbf{m}|=m_{1}+m_{2}+\cdots+m_{n}$.

We make symmetrization and antisymmetrization of the functions

$$
\mathcal{H}_{\mathbf{m}}(\mathbf{x}):=e^{-\pi|x|} H_{\mathbf{m}}(\sqrt{2 \pi} \mathbf{x})
$$

(obtained from (13.6) by replacing $\mathbf{x}$ by $\sqrt{2 \pi} \mathbf{x}$ ) by means of orbit functions of $A_{n-1}$ :

$$
\begin{aligned}
& \int_{\mathbb{R}^{n}} \hat{\phi}_{\lambda}(\mathbf{x}) e^{-\pi|\mathbf{x}|^{2}} H_{\mathbf{m}}(\sqrt{2 \pi} \mathbf{x})=\mathrm{i}^{-|\mathbf{m}|} e^{-\pi|\lambda|^{2}} H_{\mathbf{m}}^{\mathrm{sym}}(\sqrt{2 \pi} \lambda), \\
& \int_{\mathbb{R}^{n}} \varphi_{\lambda}(\mathbf{x}) e^{-\pi|\mathbf{x}|^{2}} H_{\mathbf{m}}(\sqrt{2 \pi} \mathbf{x})=\mathrm{i}^{-|\mathbf{m}|} e^{-\pi|\lambda|^{2}} H_{\mathbf{m}}^{\text {anti }}(\sqrt{2 \pi} \lambda),
\end{aligned}
$$

where $\hat{\phi}_{\lambda}(\mathbf{x})$ is a symmetric orbit function of $A_{n-1}$, given by formula (6.2) in [1], $\varphi_{\lambda}(\mathbf{x})$ is an antisymmetric orbit function of $A_{n-1}$, and $\lambda=\left(\lambda_{1}, \lambda_{2}, \ldots, \lambda_{n}\right)$.

The polynomials $H_{\mathbf{m}}^{\text {sym }}$ and $H_{\mathbf{m}}^{\text {anti }}$ are symmetric and antisymmetric, respectively, with respect to the Weyl group $W \equiv S_{n}$ of $A_{n-1}$ :

$$
H_{\mathbf{m}}^{\mathrm{sym}}(w \lambda)=H_{\mathbf{m}}^{\mathrm{sym}}(\lambda), \quad H_{\mathbf{m}}^{\mathrm{anti}}(w \lambda)=(\operatorname{det} w) H_{\mathbf{m}}^{\mathrm{anti}}(\lambda), \quad w \in S_{n} .
$$

For this reason, $H_{\mathbf{m}}^{\mathrm{sym}}(\lambda)$ are uniquely determined by their values of $\lambda=\left(\lambda_{1}, \lambda_{2}, \ldots, \lambda_{n}\right)$ such that $\lambda_{1} \geq \lambda_{2} \geq \cdots \geq \lambda_{n}$ and $H_{\mathbf{m}}^{\text {anti }}(\lambda)$ by their values of $\lambda$ such that $\lambda_{1}>\lambda_{2}>\cdots>\lambda_{n}$. (Note that $H_{\mathbf{m}}^{\text {anti }}(\lambda)=0$ if $\lambda_{i}=\lambda_{i+1}$ for some $i=1,2, \ldots, n-1$.)

The polynomials $H_{\mathbf{m}}^{\text {sym }}$ are of the form

$$
H_{\mathbf{m}}^{\mathrm{sym}}(\lambda)=\sum_{w \in S_{n}} H_{w \mathbf{m}}(\lambda)
$$

where the polynomials $H_{w \mathbf{m}}(\lambda)$ are of the form (13.5). The polynomials $H_{\mathbf{m}}^{\text {anti }}$ are of the form

$$
H_{\mathbf{m}}^{\mathrm{anti}}(\lambda)=\sum_{w \in S_{n}}(\operatorname{det} w) H_{w \mathbf{m}}(\lambda)
$$

that is,

$$
H_{\mathbf{m}}^{\operatorname{anti}}(\lambda)=\operatorname{det}\left(H_{m_{i}}\left(\lambda_{j}\right)\right)_{i, j=1}^{n} .
$$

Moreover, $H_{\mathrm{m}}^{\text {anti }}(\lambda)=0$ if $m_{i}=m_{i+1}$ for some $i=1,2, \ldots, n-1$. For this reason, we may consider the polynomials $H_{\mathbf{m}}^{\text {sym }}(\lambda)$ for $n$-tuples $\mathbf{m}$ such that $m_{1} \geq m_{2} \geq \cdots \geq m_{n}$ and the polynomials $H_{\mathbf{m}}^{\text {anti }}(\lambda)$ for $n$-tuples $\mathbf{m}$ such that $m_{1}>m_{2}>\cdots>m_{n}$.

Let us apply symmetric orbit function transform (8.10) of [1] to the symmetric function (13.10). Taking into account formula (13.8) we obtain

$$
\begin{aligned}
\mathfrak{F}\left(e^{-\pi|\mathbf{x}|^{2}} H_{\mathbf{m}}^{\mathrm{sym}}(\sqrt{2 \pi} \mathbf{x})\right) & :=\frac{1}{\left|S_{n}\right|} \int_{\mathbb{R}^{n}} \hat{\phi}_{\lambda}(\mathbf{x}) e^{-\pi|\mathbf{x}|^{2}} H_{\mathbf{m}}^{\mathrm{sym}}(\sqrt{2 \pi} \mathbf{x}) d \mathbf{x} \\
& =\mathrm{i}^{-|\mathbf{m}|} e^{-\pi|\lambda|^{2}} H_{\mathbf{m}}^{\mathrm{sym}}(\sqrt{2 \pi} \lambda),
\end{aligned}
$$

where $\left|S_{n}\right|$ is an order of the permutation group $S_{n}$, that is, functions (13.10) are eigenfunctions of the symmetric orbit function transform $\mathfrak{F}$. Since the functions $(13.10)$ for $m_{i}=0,1,2, \ldots$, 
$i=1,2, \ldots, n, m_{1} \geq m_{2} \geq \cdots \geq m_{n}$, form an orthogonal basis of the Hilbert space $L_{\text {sym }}^{2}\left(\mathbb{R}^{n}\right)$ of functions from $L^{2}\left(\mathbb{R}^{n}\right)$ symmetric with respect to $W$, then they constitute a complete set of eigenfunctions of this transform. Thus, this transform has only four eigenvalues $i,-i, 1,-1$ in $L_{\mathrm{sym}}^{2}\left(\mathbb{R}^{n}\right)$. This means that, as in the case of the usual Fourier transform, we have

$$
\mathfrak{F}^{4}=1
$$

Now we apply antisymmetric orbit function transform (10.12) to the antisymmetric function (13.11). Taking into account formula (13.9) we obtain

$$
\begin{aligned}
\tilde{\mathfrak{F}}\left(e^{-\pi|\mathbf{x}|^{2}} H_{\mathbf{m}}^{\text {anti }}(\sqrt{2 \pi} \mathbf{x})\right) & :=\frac{1}{\left|S_{n}\right|} \int_{\mathbb{R}^{n}} \varphi_{\lambda}(\mathbf{x}) e^{-\pi|\mathbf{x}|^{2}} H_{\mathbf{m}}^{\text {anti }}(\sqrt{2 \pi} \mathbf{x}) d \mathbf{x} \\
& =\mathrm{i}^{-|\mathbf{m}|} e^{-\pi|\lambda|^{2}} H_{\mathbf{m}}^{\text {anti }}(\sqrt{2 \pi} \lambda), \quad m_{1}>m_{2}>\cdots>m_{n} \geq 0,
\end{aligned}
$$

that is, functions (13.11) are eigenfunctions of the symmetric orbit function transform $\tilde{\mathfrak{F}}$. Since the functions (13.11) for $m_{i}=0,1,2, \ldots ; i=1,2, \ldots, n, m_{1}>m_{2}>\cdots>m_{n} \geq 0$, form an orthogonal basis of the Hilbert space $L_{\text {anti }}^{2}\left(\mathbb{R}^{n}\right)$ of functions from $L^{2}\left(\mathbb{R}^{n}\right)$ antisymmetric with respect to $W$, then they constitute a complete set of eigenfunctions of this transform. Thus, this transform has only four eigenvalues $\mathrm{i},-\mathrm{i}, 1,-1$. This means that, as in the case of the usual Fourier transform, we have

$$
\tilde{\mathfrak{F}}^{4}=1 .
$$

\subsection{Symmetric and antisymmetric sets of polynomials}

In the previous subsection we constructed symmetric and antisymmetric sets of functions connected with Hermite polynomials. Similarly other sets of orthogonal polynomials can be constructed (see [37, 38] and [39]).

Let $p_{m}(x), m=0,1,2, \ldots$, be the set of orthogonal polynomials in one variable such that

$$
\int_{\mathbb{R}} p_{m}(x) p_{m^{\prime}}(x) d \sigma(x)=\delta_{m m^{\prime}}
$$

where $d \sigma(x)$ is some orthogonality measure, which may be finite or discrete.

We create a set of symmetric polynomials of $n$ variables as follows:

$$
\begin{aligned}
& p_{\mathbf{m}}^{\mathrm{sym}}(\mathbf{x})=\sum_{w \in S_{n} / S_{\mathbf{m}}} p_{m_{w(1)}}\left(x_{1}\right) p_{m_{w(2)}}\left(x_{2}\right) \cdots p_{m_{w(n)}}\left(x_{n}\right), \\
& m_{i}=0,1,2, \ldots, \quad i=1,2, \ldots, n
\end{aligned}
$$

where $\mathbf{m}=\left(m_{1}, m_{2}, \ldots, m_{n}\right), m_{1} \geq m_{2} \geq \cdots \geq m_{n} \geq 0, \mathbf{x}=\left(x_{1}, x_{2}, \ldots, x_{n}\right)$, and $w(1), w(2)$, $\ldots, w(n)$ is a set of numbers $1,2, \ldots, n$ transformed by the permutation $w \in S_{n} / S_{\mathbf{m}}$, where $S_{\mathbf{m}}$ is the subgroup of $S_{n}$ consisting of elements leaving $\mathbf{m}$ invariant.

We also create the set of polynomials

$$
\begin{aligned}
& p_{\mathbf{m}}^{\operatorname{anti}}(\mathbf{x})=\sum_{w \in S_{n}}(\operatorname{det} w) p_{m_{w(1)}}\left(x_{1}\right) p_{m_{w(2)}}\left(x_{2}\right) \cdots p_{m_{w(n)}}\left(x_{n}\right)=\operatorname{det}\left(p_{m_{i}}\left(x_{j}\right)\right)_{i, j=1}^{n}, \\
& m_{i}=0,1,2, \ldots, \quad i=1,2, \ldots, n
\end{aligned}
$$

where notations are the same as in (13.12) and the condition $m_{1}>m_{2}>\cdots>m_{n} \geq 0$ is satisfied. 
It is easy to check that the polynomials $p_{\mathbf{m}}^{\mathrm{sym}}(\mathbf{x})$ are symmetric with respect to transformations of $S_{n}$ :

$$
p_{\mathbf{m}}^{\mathrm{sym}}(w \mathbf{x})=p_{\mathbf{m}}^{\mathrm{sym}}(\mathbf{x}), \quad w \in S_{n} .
$$

Similarly, the polynomials $p_{\mathbf{m}}^{\text {anti }}(\mathbf{x})$ are antisymmetric with respect to transformations of $S_{n}$ :

$$
p_{\mathbf{m}}^{\operatorname{anti}}(w \mathbf{x})=(\operatorname{det} w) p_{\mathbf{m}}^{\operatorname{anti}}(\mathbf{x}), \quad w \in S_{n}
$$

Thus, we may consider the polynomials (13.12) and (13.13) on the closure of the fundamental domain of the transformation group $W\left(A_{n-1}\right) \equiv S_{n}$. This closure (we denote it by $\overline{D_{+}}$) coincides with the set of points $\mathbf{x}=\left(x_{1}, x_{2}, \ldots, x_{n}\right)$ for which

$$
x_{1} \geq x_{2} \geq \cdots \geq x_{n} \geq 0 .
$$

The polynomials (13.13) vanish if for some $i, i=1,2, \ldots, n-1$, we have $x_{i}=x_{i+1}$.

The set of polynomials (13.12), as well as the set of the polynomials (13.13), is orthogonal with respect to the product measure $d \sigma(\mathbf{x}) \equiv d \sigma\left(x_{1}\right) d \sigma\left(x_{2}\right) \cdots d \sigma\left(x_{n}\right)$. Indeed, we have

$$
\begin{aligned}
& \int_{\overline{D_{+}}} p_{\mathbf{m}}^{\mathrm{sym}}(\mathbf{x}) \overline{p_{\mathbf{m}^{\prime}}^{\mathrm{sym}}(\mathbf{x})} d \sigma(\mathbf{x})=\frac{|O(\mathbf{m})|}{\left|S_{n}\right|} \delta_{\mathbf{m m}^{\prime}}=\frac{1}{\left|S_{\mathbf{m}}\right|} \delta_{\mathbf{m m}^{\prime}}, \\
& \int_{\overline{D_{+}}} p_{\mathbf{m}}^{\text {anti }}(\mathbf{x}) \overline{p_{\mathbf{m}^{\prime}}^{\text {anti }}(\mathbf{x})} d \sigma(\mathbf{x})=\delta_{\mathbf{m m}^{\prime}}
\end{aligned}
$$

where $O(\mathbf{m})$ is the $S_{n}$-orbit of the point $\mathbf{m}$.

Note that each polynomial $p_{\mathbf{m}}^{\text {anti }}(\mathbf{x})$ vanishes at $x_{i}=x_{j}$ for any admitted $i$ and $j$. This means that $p_{\mathbf{m}}^{\text {anti }}(\mathbf{x})$ can be divided by $x_{i}-x_{j}$. Therefore, the functions

$$
P_{\mathbf{m}}^{\mathrm{anti}}(\mathbf{x})=\frac{p_{\mathbf{m}}^{\mathrm{anti}}(\mathbf{x})}{\prod_{1 \leq i<j \leq n}\left(x_{i}-x_{j}\right)}
$$

are polynomials in $x_{i}, i=1,2, \ldots, n$. These polynomials are also orthogonal and the orthogonality relation is of the form

$$
\int_{\overline{D_{+}}} P_{\mathbf{m}}^{\mathrm{anti}}(\mathbf{x}) \overline{P_{\mathbf{m}^{\prime}}^{\text {anti }}(\mathbf{x})} \Xi(\mathbf{x}) d \sigma(\mathbf{x})=\delta_{m m^{\prime}}
$$

where $\Xi(\mathbf{x})=\prod_{1 \leq i<j \leq n}\left(x_{i}-x_{j}\right)^{2}$.

\section{Acknowledgements}

The first author (AK) acknowledges CRM of University of Montreal for hospitality when this paper was under preparation. His research was partially supported by Grant 10.01/015 of the State Foundation of Fundamental Research of Ukraine. We are grateful for partial support for this work to the National Research Council of Canada, MITACS, the MIND Institute of Costa Mesa, California, and Lockheed Martin, Canada. 


\section{References}

[1] Klimyk A.U., Patera J., Orbit functions, SIGMA 2 (2006), 006, 60 pages, math-ph/0601037.

[2] Patera J., Orbit functions of compact semisimple Lie groups as special functions, in Proceedings of Fifth International Conference "Symmetry in Nonlinear Mathematical Physics" (June 23-29, 2003, Kyiv), Editors A.G. Nikitin, V.M. Boyko, R.O. Popovych and I.A. Yehorchenko, Proceedings of Institute of Mathematics, Kyiv 50 (2004), Part 3, 1152-1160.

[3] Macdonald I.G., Symmetric functions and Hall polynomials, 2nd ed., Oxford University Press, Oxford, 1995.

[4] Macdonald I.G., A new class of symmetric functions, Publ. I.R.M.A. Strasbourg, 372/S-20, Actes 20 Séminaire Lotharingien, 1988, 131-171.

[5] Macdonald I.G., Orthogonal polynomials associated with root systems, Séminaire Lotharingien de Combinatoire, Actes B45a, Stracbourg, 2000.

[6] Vilenkin N.Ja., Klimyk A.U., Representations of Lie groups and special functions: recent advances, Kluwer, Dordrecht, 1995.

[7] Moody R.V., Patera J., Elements of finite order in Lie groups and their applications, in Proceedings of XIII Int. Colloq. on Group Theoretical Methods in Physics, Editor W. Zachary, World Scientific Publishers, Singapore, 1984, 308-318.

[8] McKay W.G., Moody R.V., Patera J., Tables of $E_{8}$ characters and decomposition of plethysms, in Lie Algebras and Related Topics, Editors D.J. Britten, F.W. Lemire and R.V. Moody, Amer. Math. Society, Providence RI, 1985, 227-264.

[9] McKay W.G., Moody R.V., Patera J., Decomposition of tensor products of $E_{8}$ representations, Algebras Groups Geom. 3 (1986), 286-328.

[10] Patera J., Sharp R.T., Branching rules for representations of simple Lie algebras through Weyl group orbit reduction, J. Phys. A: Math. Gen. 22 (1989), 2329-2340.

[11] Grimm S., Patera J., Decomposition of tensor products of the fundamental representations of $E_{8}$, in Advances in Mathematical Sciences - CRM's 25 Years, Editor L. Vinet, CRM Proc. Lecture Notes, Vol. 11, Amer. Math. Soc., Providence, RI, 1997, 329-355.

[12] Rao K. R., Yip P., Disrete cosine transform - algorithms, advantages, applications, Academic Press, New York, 1990.

[13] Atoyan A., Patera J., Properties of continuous Fourier extension of the discrete cosine transform and its multidimensional generalization, J. Math. Phys. 45 (2004), 2468-2491, math-ph/0309039.

[14] Patera J., Zaratsyan A., Discrete and continuous cosine transform generalized to Lie groups $S U(2) \times S U(2)$ and $O(5)$, J. Math. Phys. 46 (2005), 053514, 17 pages.

[15] Patera J., Zaratsyan A., Discrete and continuous cosine transform generalized to Lie groups $S U(2)$ and $G_{2}$, J. Math. Phys. 46 (2005), 113506, 25 pages.

[16] Atoyan A., Patera J., Continuous extension of the discrete cosine transform, and its applications to data processing, in Group Theory and Numerical Analysis, CRM Proc. Lecture Notes, Vol. 39, Amer. Math. Soc., Providence, RI, 2005, 1-15.

[17] Atoyan A., Patera J., Sahakian V., Akhperjanian A., Fourier transform method for imaging atmospheric Cherenkov telescopes, Astroparticle Phys. 23 (2005), 79-95, astro-ph/0409388.

[18] Patera J., Zaratsyan A., Zhu H.-M., New class of interpolation methods based on discretized Lie group transform, in SPIE Electronic Imaging, 2006, 6064A-06, S1.

[19] Germain M., Patera J., Zaratsyan A., Multiresolution analysis of digital images using the continuous extension of discrete group transform, in SPIE Electronic Imaging, 2006, 6065A-03, S2.

[20] Germain M., Patera J., Allard Y., Cosine transform generalized to Lie groups $S U(2) \times S U(2), O(5)$, and $S U(2) \times S U(2) \times S U(2)$ : application to digital image processing, Proc. SPIE 6065 (2006), 387-395.

[21] Moody R.V., Patera J., Computation of character decompositions of class functions on compact semisimple Lie groups, Math. Comp. 48 (1987), 799-827.

[22] Patera J., Zaratsyan A., Discrete and continuous sine transforms generalized to compact semisimple Lie groups of rank two, J. Math. Phys. 47 (2006), 043512, 22 pages.

[23] Kashuba I., Patera J., Discrete and continuous exponential transforms of simple Lie groups of rank two, math-ph/0702016.

[24] Kane R., Reflection groups and invariants, Springer, New York, 2002. 
[25] Humphreys J.E., Reflection groups and Coxeter groups, Cambridge University Press, Cambridge, 1990.

[26] Humphreys J.E., Introduction to Lie algebras and representation theory, Springer, New York, 1972.

[27] Bremner M.R., Moody R.V., Patera J., Tables of dominant weight multiplicities for representations of simple Lie algebras, Marcel Dekker, New York, 1985.

[28] Kac V., Infinite dimensional Lie algebras, Birkhäuser, Basel, 1982.

[29] Patera J., Compact simple Lie groups and their $C$-, $S$-, and E-transforms, SIGMA 1 (2005), 025, 6 pages, math-ph/0512029.

[30] Weyl H., The classical groups, Princeton University Press, 1939.

[31] Moody R.V., Patera J., Orthogonality within the families of $C$-, $S$-, and E-functions of any compact semisimple Lie group, SIGMA 2 (2006), 076, 14 pages, math-ph/0611020.

[32] Mckay W.G., Patera J., Sannikoff D., The computation of branching rules for representations of semisimple Lie algebras, in Computers in Nonassociative Rings and Algebras, Editors R.E. Beck and B. Kolman, Academic Press, New York, 1977, 235-278.

[33] Zhelobenko D.P., Compact Lie groups and their representations, Nauka, Moscow, 1970.

[34] Strang G., The discrete cosine transform, SIAM Rev. 41 (1999), 135-147.

[35] Martuchi S.A., Symmetric convolution and the discrete sine and cosine transforms, IEEE Trans. Signal Process. 42 (1994), 1038-1051.

[36] Vilenkin N.Ja., Klimyk A.U., Representations of Lie groups and special functions, Vol. 2, Kluwer, Dordrecht, 1993.

[37] Karlin S., McGregor J., Determinants of orthogonal polynomials, Bull. Amer. Math. Soc. 68 (1962), 204209.

[38] Koornwinder T., Two-variable analogues of the classical orthogonal polynomials, in Theory and Applications of Special Functions, Editor R.A. Askey, Academic Press, New York, 1975, 435-495.

[39] Berens H., Schmid H., Xu Y., Multivariate Gaussian cubature formulas, Arch. Math. 64 (1995), 26-32. 